\title{
A Survey on Mobile Crowdsensing Systems: Challenges, Solutions and Opportunities
}

\author{
Andrea Capponi, Student Member, IEEE, Claudio Fiandrino, Member, IEEE, Burak Kantarci, Senior Member, IEEE, \\ Luca Foschini, Senior Member, IEEE, Dzmitry Kliazovich, Senior Member, IEEE, and Pascal Bouvry, Member, IEEE
}

\begin{abstract}
Mobile crowdsensing (MCS) has gained significant attention in recent years and has become an appealing paradigm for urban sensing. For data collection, MCS systems rely on contribution from mobile devices of a large number of participants or a crowd. Smartphones, tablets, and wearable devices are deployed widely and already equipped with a rich set of sensors, making them an excellent source of information. Mobility and intelligence of humans guarantee higher coverage and better context awareness if compared to traditional sensor networks. At the same time, individuals may be reluctant to share data for privacy concerns. For this reason, MCS frameworks are specifically designed to include incentive mechanisms and address privacy concerns. Despite the growing interest in the research community, MCS solutions need a deeper investigation and categorization on many aspects that span from sensing and communication to system management and data storage. In this paper, we take the research on MCS a step further by presenting a survey on existing works in the domain and propose a detailed taxonomy to shed light on the current landscape and classify applications, methodologies and architectures. Our objective is not only to analyze and consolidate past research but also to outline potential future research directions and synergies with other research areas.
\end{abstract}

Index Terms-Mobile crowdsensing, urban sensing, opportunistic sensing, participatory sensing.

\section{INTRODUCTION}

$\mathbf{M}$ OBILE crowdsensing (MCS) has gained popularity in recent years becoming an appealing paradigm for sensing and collecting data. MCS systems rely on sensors and communication interfaces embedded in commonly used mobile devices such as smartphones and wearables. Nowadays, mobile devices are essential for our daily activities, including business, communication, and entertainment [1], [2]. According to Gartner statistics, the number of worldwide smartphones sales in 2018 was 1.55 billion units [3], and the number of wearable devices shipped in 2018 was 178.91 million, which is projected to reach 453.19 million in 2022 [4]. Smart watches, glasses, rings, gloves, and helmets are the most popular wearable devices currently available on the market corresponding to a highly increasing revenue that is estimated to

A. Capponi and P. Bouvry are with the University of Luxembourg, Luxembourg. E-mail: firstname.lastname@uni.lu

C. Fiandrino is with Imdea Networks Institute, Madrid, Spain. E-mail: claudio.fiandrino@imdea.org

B. Kantarci is with the School of Electrical Engineering and Computer Science, University of Ottawa, Ottawa, ON, Canada. E-mail: burak.kantarci@uOttawa.ca

L. Foschini is with DISI, Univ. Bologna, Viale Risorgimento 2, 40136 Bologna, Italy. E-mail: luca.foschini@unibo.it

D. Kliazovich is with ExaMotive, Luxembourg. E-mail: kliazovich@ieee.org. rise up to USD 95.3 billion by 2021 [5]. Furthermore, the crowd analytics market is predicted to reach USD 1142.5 million by 2021 raising from USD 385.1 million of 2016 at a compound annual growth rate of $24.3 \%$ [6].

The term mobile crowdsensing was first introduced by Ganti et al. to indicate a more general paradigm [7] than mobile phone sensing [8], [9]. Guo et al. in [10] give a definition that clearly highlights this difference: "MCS is a new sensing paradigm that empowers ordinary citizens to contribute data sensed or generated from their mobile devices, aggregates and fuses the data in the cloud for crowd intelligence extraction and people-centric service delivery". To operate efficiently, MCS systems require the participation and contribution of a large number of users. Although entire communities can potentially benefit from such a contribution, a singular person may be reluctant to participate, being selfish or having privacy concerns. To ease this burden, in the last years the research community has put lots of effort in developing proper incentive mechanisms [11]-[14] and in investigating privacy issues [15], [16].

The capillary spread of smartphones and wearables along with the rich set of built-in sensors are certainly the main key enablers leading to the success of MCS paradigm. Accelerometer, gyroscope, GPS, microphone, and camera are only a representative set of sensors that facilitated the development of several applications in a wide range of scenarios, including health care, environmental, and traffic monitoring. Many applications using smartphone sensors have been already developed and are currently in use. To illustrate representative examples, HealthAware [17], MPCS [18], and DietSense [19] foster healthy eating by collecting images of consumed food and inspect daily user-activity by extracting context information such as time and location where food was consumed. For this purpose, both applications use the accelerometer, GPS, and microphone. Nericell [20] monitors traffic conditions. GasMobile [21], HazeWatch [22], and ThirdEye [23] rely on active citizen participation to monitor air pollution. Creekwatch [24], developed by the IBM Almaden research center, permits to monitor the conditions of the watershed through crowdsensed collected data about the amount of water in the river bed, the amount of trash in the river bank, the flow rate, and a picture of the waterway. Garbage Watch [25] and WasteApp [26] allow monitoring the content of recycling bins with the objective to improve the recycling program.

MCS can significantly improve citizens everyday life and provide new perspectives to urban societies. MCS is an essential solution for building smart cities of the future, which 


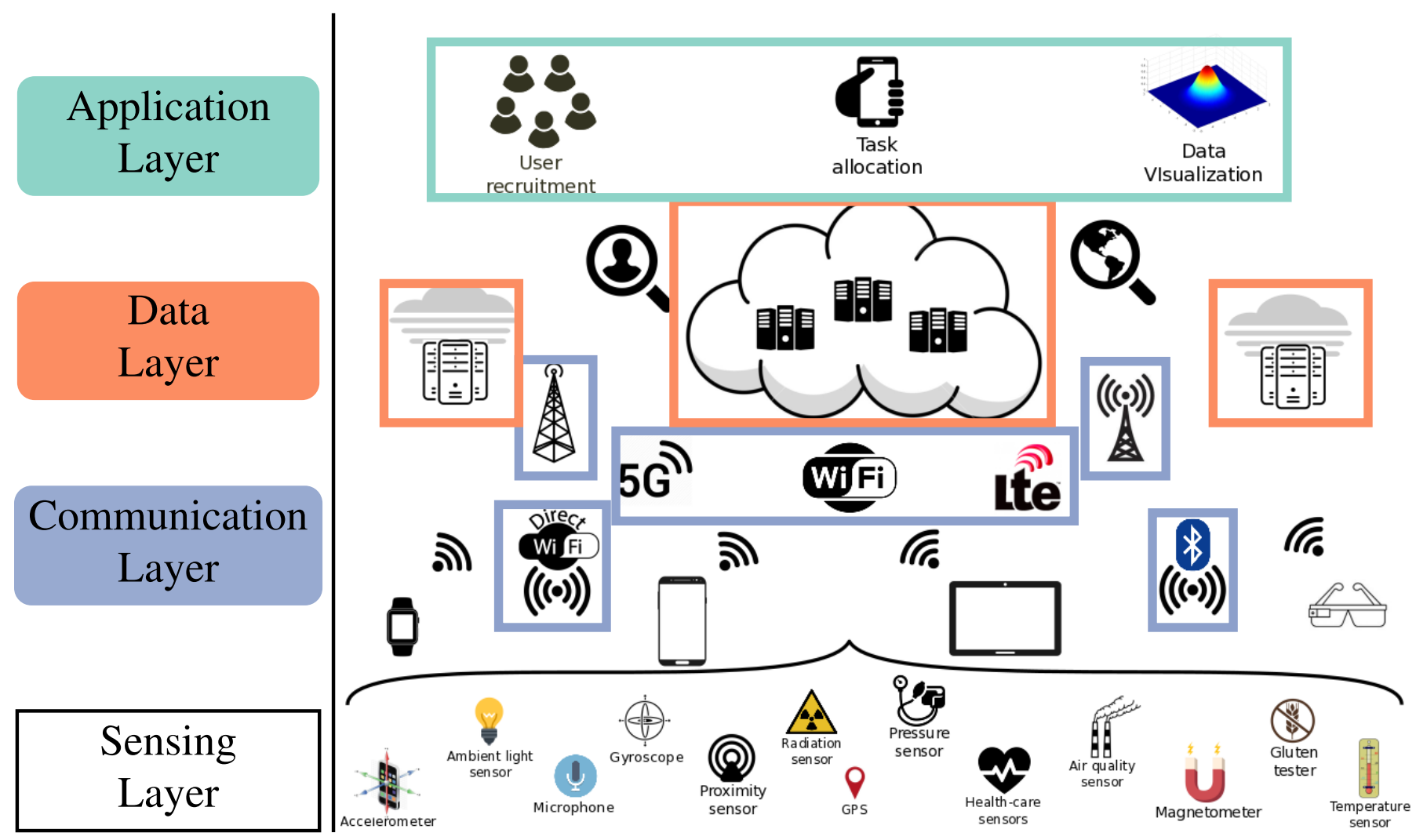

Fig. 1. Layered architecture of MCS systems. It shows the four-layered architecture that describes the MCS paradigm, including application (Fig. 5), data (Fig. 6), communication (Fig. 7), and sensing (Fig. 8) layers, which are discussed in Sec. III-A.

aim at using ICT solutions to improve the management of everyday life of their citizens [27], [28]. The Internet of Things (IoT) paradigm is the candidate solution for a wide deployment of sensing infrastructure enabling smart cities' applications [29]. Moreover, active participation of citizens can improve spatial coverage of already deployed sensing systems with no need for further investments. MCS leverages human intelligence, which has a deeper context understanding than traditional sensor networks. Cities are facing significant deficits in infrastructure services and humans can be involved to improve their monitoring and maintenance. We illustrate this concept with specific use cases. Data harvested from smartphones' accelerometers over moving vehicles enables the detection of bridge vibrations [30]. Other possible city services where MCS plays a fundamental role are smart traffic management [31], [32] or free parking spot detection. Specifically, ParkSense detects vacant parking spots using WiFi scans of smartphones [33], while ParkGauge reports real-time crowdsensed information about indoor parking occupancy and exploits low-consuming sensors (e.g., accelerometer) to detect the driving states [34].

There is a wealth of literature on MCS systems. Considerable research efforts proposed novel sensing architectures and, as already mentioned, investigated specific aspects such as incentive mechanisms, privacy issues as well as the reliability and trust of the data collection process. With such a large body of work, surveys on the topic covering specific areas, such as privacy [15] or incentives for user recruitment [12], [13] already exists. Others, like [35], present many aspects components of MCS as an emerging paradigm, e.g., data collection, quality assurance, etc. However, this is an early work missing a wealth of early developments in the field. What is missing in this picture is a survey that covers the whole decade of research in MCS and provides a clear state of its evolution. This is one of the purposes of our manuscript. Also, the vast amount of work in literature remains uncategorized, with many of the core paradigms unclear. To illustrate, for example, there is no consensus on the term "opportunistic sensing". According to Ganti et al. [7] "opportunistic sensing" is defined as "On the other hand, opportunistic sensing is where the sensing is more autonomous and user involvement is minimal (e.g. continuous location sampling)". However, Khan et al. [9] state that opportunistic sensing requires no user involvement at all, since the decisions to perform the sensing are a prerogative of the device itself. Finally, Han et al. [36] enlarge the previous vision of opportunistic sensing in the context of single-user involvement and they describe opportunistic sensing as a paradigm enabling cooperation among smartphones. Typically, both terms "opportunistic" and "participatory" sensing remain under the common umbrella of MCS [7], [9], [36], [37]. In other cases, both "mobile crowdsensing" and "participatory sensing" are used interchangeably [31]. Other times, both "mobile crowdsensing", "participatory sensing", and "opportunistic sensing" are synonyms [38].

With this plethora of definitions, our manuscript has the precise objective of proposing detailed taxonomies to sim- 

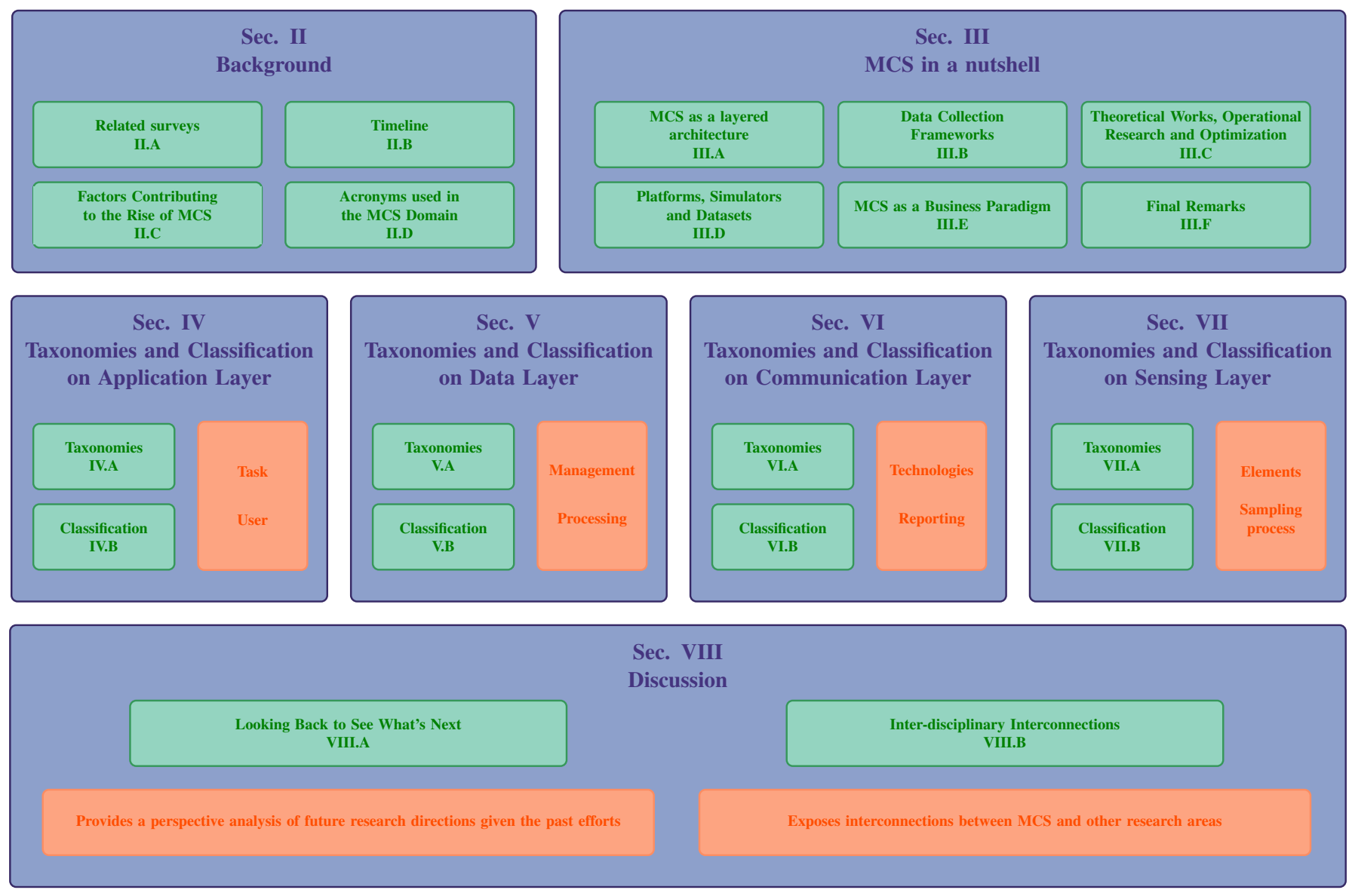

Sec. IX

Concluding Remarks

Fig. 2. Survey organization. Section II provides a background on MCS literature. Section III presents the four-layered architecture, and discusses theoretical and practical works. Section IV, V, VI, and VII propose taxonomies and classification on the four layers, i.e., application, data, communication, and sensing layers. Section VIII discusses future directions and interconnections with other research areas. Finally, Section IX concludes the survey.

plify the understanding of the current definitions, available techniques, and solutions in the field of MCS. We foresee to categorize MCS works in a four-layered architecture, as shown in Fig. 1. The underneath reason, better detailed after that, is to cover the entire process chain since the sensor produces a reading up to when data reaches the application layer. The four-layered architecture is structured as follows. The top layer is the application layer, which involves high-level functionalities such as user recruitment and task allocation [39]. The second is the data layer, responsible for aspects related to store, analyze, and process collected information. The third layer involves the communication layer, comprising the communication technologies for the delivery of sensed data. Finally, the bottom layer close to the physical layer is the sensing layer. Our approach goes beyond such classification by proposing specific taxonomies for each layer of the architecture.

Specifically, the synopsis of contributions of the current survey is as follow:

- To introduce MCS as a four-layered architecture, divided into application, data, communication, and sensing layers.

- To compare and analyze existing MCS data collection frameworks (DCFs), theoretical works leveraging operational research and optimization, practical ones such as platforms, simulators, and those making crowdsensed datasets publicly available.

- To propose novel and detailed taxonomies based on the layered architecture that cover all MCS aspects, allowing for a simple and clear classification of MCS systems and domains.

- To classify MCS systems according to the proposed taxonomies.

- To discuss future directions given the consolidated past efforts and inter-disciplinary research areas.

Survey organization. Fig. 2 shows the organization of this survey. Section II provides a background on related surveys and a timeline including the most relevant works, followed by the most important technological factors and related fields that have contributed to the rise of MCS and finally lists acronyms used in the MCS domain. Section III presents MCS in a nutshell, introducing it as a four-layered architecture, presenting its main data collection frameworks (DCFs), discussing theoretical 
works on operational research and optimization problems, but also more practical ones about platforms and simulators exploited for crowdsensing campaigns and collected datasets. Then, it discusses MCS as a business paradigm and concludes with final remarks, summarizing previous contents and introducing taxonomies based on the presented layered architecture. Section IV elaborates on novel taxonomies on the application layer and overviews papers accordingly. Section V proposes novel taxonomies on the data layer and surveys existing solutions according to the presented taxonomies. Section VI discusses novel taxonomies on the communication layer and consequently overviews works. Section VII presents novel taxonomies on the sensing layer and classifies MCS systems accordingly. Section VIII provides an overall discussion on the subject, by presenting a prospective analysis of future research directions given the past efforts and inter-disciplinary interconnections with other research areas. Finally, Section IX concludes the survey.

\section{BACKGROUND}

This section explores the motivations giving rise to MCS and the reasons that make it a prominent sensing paradigm. To this end, we analyze and extract from the large body of literature the works that can be defined as milestones, i.e., the works that have strongly influenced the research in the field. This analysis considers the evolution of technologies in different domains such as computing and communications, which significantly affect the efficiency of the data collection process. Specifically, we overview in Section II-A related surveys to guide the reader into past research, and in Section II-B we show the temporal evolution of milestones works in the area. We conclude the section by providing an overview of the main factors contributing to the rise of MCS (Section II-C) and present how MCS services are delivered (Section II-D).

\section{A. Related Surveys}

This Section presents surveys that relate with concepts of MCS (see Table I). We categorize these works into six categories: surveys on MCS (i.e., those about sensing equipment), those analyzing wireless sensor networks, mobile phone sensing, anticipatory mobile computing, user recruitment, and privacy issues.

Mobile Crowdsensing Guo et al. [35] coin the term Mobile Crowd Sensing and Computing (MCSC), which investigates the complementary nature of the machine and human intelligence in sensing and computing processes. Citizens contribute data with their mobile devices to the cloud which enforces crowd intelligence. Guo et al. also introduce the concept of visual crowdsensing in [40], by presenting its strengths and challenges, like multi-dimensional coverage needs, low-cost transmission and data redundancy. In [42] provides a discussion on the implementation requirements of MCS systems. Like our proposal, the focus is on four dimensions task assignment, stimulation of the participation, data collection, and processing. Liu et al. survey the challenges of MCS systems and the proposed solutions for the effective use of resources [43]. An overview of different applications of MCS systems in the context of IoT and smart cities is provided in [44]. In [41], the authors focus on citizens as data consumers and data producers to infer social relationships and human activities. In particular, they overview applications on social sensors based on social sensor receiver platform (e.g., Twitter) and present a classification, including public security, smart city, and location-based services. Crowdsensed data can be malicious or unreliable. Despite assessing Quality of Information (QoI) is important, few works delved into the roots of the problem. In [54], the authors overview existing works assessing QoI and propose a framework to enforce it.

Sensors \& WSN Sensors are an essential component for data acquisition. Sensing equipment is typically embedded in mobile devices, but specific applications (e.g., air quality monitoring [21], [55], [56]) can employ dedicated hardware connected with wireless technology (e.g., Bluetooth). Ming presents a study of mobile sensing [48], by providing an overview of typical sensors embedded in smartphones and discussing related mobile applications. The actors in a crowdsensing campaign can be seen as nodes of a Wireless Sensor Network (WSN). In the last years, developments in communication technologies and electronics lead to the significant progress of sensor networks and typically survey in the area analyze both sensing and networking aspects [45], [47], and [46]. Remote sensing technologies are discussed in [49], where the authors present platform and sensor developments for emerging new remote sensing satellite constellations, sensor geo-referencing and supporting navigation infrastructure.

Mobile Phone Sensing Mobile phone sensing can be seen as the forefather of mobile crowdsensing. This paradigm was very popular when mobile phones did not have the capabilities of current smartphones in terms of storage, communication, and computation. Unlike in MCS, the research on mobile phone sensing focused mostly on individual sensing applications, such as elderly fall detection or personal well-being. Several works exploit this paradigm, proposing methodologies and solutions collecting data from sensors of mobile phones [8], [9].

Anticipatory mobile computing \& networking The rich data availability enables the possibility to infer and predict context and user behavior. In [50], the authors overview the literature in mobile sensing and prediction focusing on the concept of anticipatory mobile computing. The survey presents a plethora of phenomena like user destination or behavior that smartphones can infer and predict by leveraging machine learning techniques and proactive decision making. In [51], the authors analyze the concept of anticipatory mobile networking to study pattern and periodicity of user behavior and network dynamics. The ultimate goal is to predict context and optimize network performance. The authors provide an in-depth overview of the most relevant prediction and optimization techniques.

User Recruitment The success of a crowdsensing campaign relies on large participation and contribution of citizens. To this end, incentives are fundamental. Existing surveys on user recruitment investigate how to propose incentive mechanisms that could efficiently and effectively motivate users in sensing 
TABLE I

RELATED SURVEYS

\begin{tabular}{|c|c|c|}
\hline TOPIC & DESCRIPTION & REFERENCES \\
\hline Mobile Crowdsensing & $\begin{array}{l}\text { Include works that survey crowdsensing architectures, frameworks and data } \\
\text { collection techniques }\end{array}$ & [35], [40], [41] [42]-[44] \\
\hline Sensors \& Sensor Networks & $\begin{array}{l}\text { Describe generic sensing equipment when employed by crowdsensing applica- } \\
\text { tions, sensor networks, and platforms in different domains. }\end{array}$ & {$[45]-[49]$} \\
\hline Mobile Phone Sensing & $\begin{array}{l}\text { Describe methodologies of employment of sensing equipment embedded in } \\
\text { mobile devices for non-crowdsensed applications. }\end{array}$ & [8], [9] \\
\hline Anticipatory Mobile Computing \& Networking & $\begin{array}{l}\text { Describe techniques like machine learning to predict the context of sensing and } \\
\text { network state. }\end{array}$ & [50], [51] \\
\hline User Recruitment & $\begin{array}{l}\text { Survey techniques to recruit users for sensing campaigns and describe existing } \\
\text { incentive mechanisms to promote participation. }\end{array}$ & {$[12],[13],[52],[53]$} \\
\hline Privacy & $\begin{array}{l}\text { Present the threats to users and privacy mechanisms that are exploited in existing } \\
\text { crowdsensing applications to address these issues. }\end{array}$ & {$[15],[16]$} \\
\hline
\end{tabular}

and reporting information. In [52], the authors overview existing incentive mechanisms in participatory sensing, while [53] proposes a taxonomy while providing application-specific examples. In [12], the authors analyze strategies to stimulate individuals in participating in a sensing process. They classify research works into three categories, namely entertainment, service, and money. The first category considers methods that stimulate participation by turning sensing tasks into games. Service-based mechanisms consist of providing services to the users in exchange for their data. Monetary incentives methods distribute funds to reward users' contribution.

Privacy Data collection through mobile devices presents many privacy breaches, such as tracking a user's location or disclosing the content of pictures or audio. In [16], the authors provide an overview of sensing applications and threats to individual privacy when personal data is acquired and disclosed. They analyze how privacy aspects are addressed in existing application scenarios, assessing the presented solutions and presenting countermeasures. Other works focus on privacy concerns in task management processes, such as user recruitment and task distribution [15].

The Novelty of This Survey This survey goes beyond the works mentioned above. The focus is to categorize the literature according to the corresponding "technological" layer, i.e., sensing, communication, data processing, and application. Such a perspective allows the reader to obtain insights on specific challenges at each layer as well as the interplay between the layers. Previous works instead, typically focus on single aspects such as user recruitment, data collection or methodologies to foster and promote privacy. This work does characterize such aspects by specifically showing the role of each with respect to each "technological" layer.

\section{B. Timeline}

This section presents the temporal evolution of the milestones works that contributed to shaping the crowdsensing paradigm. Fig. 3 shows graphically the time of appearance and groups the research works into four categories:

- Mobile phone sensing, crowdsourcing, and anticipatory computing;

- Seminal works;

- Simulators and platforms;

\section{- Privacy and trust.}

To uncover the time-evolution, the following paragraphs describe these fundamental works by year.

2006. The concept of crowdsourcing appeared originally in an article written by Howe [57], that describes the rise of this paradigm giving an exact definition and presenting some of the first applications in this field. Burke et al. introduced participatory sensing as a novel paradigm in which people use their mobile devices to gather and share local knowledge [58].

2007. The use of mobile devices for automatic multimedia collection for specific application appeared with DietSense, where image browsing, processing, and clustering are exploited to manage health care and nutrition in participatory sensing [19].

2008. Miluzzo et al. introduced the CenceMe application, which is among the first systems that combine data collection from sensors embedded in mobile devices with sharing on social networks [59]. Micro-blog is one of the first applications which aims at sharing and querying content through mobile phones and social participation. Users are encouraged to record multimedia blogs that may be enriched with a variety of sensor data [60].

2010. Lane et al. presented a survey about mobile phone sensing, which includes a detailed description of sensors and existing applications. Furthermore, the article shows how to scale from personal sensing to community sensing through data aggregation [8].

2011. Ganti et al. proposed one among the first surveys that are specific on MCS. They uncover the potential of the crowd, where individuals with sensing and computing devices collectively share data to measure and map phenomena of common interest [7]. Christin et al. presented one among the first works analyzing the state-of-art in privacy-related concerns of participatory sensing systems [16].

2013. Cardone et al. investigate how to foster the participation of citizens through a geo-social crowdsensing platform [61]. The article focuses on three main technical aspects, namely a geo-social model to profile users, a matching algorithm for participant selection and an Android-based platform to acquires data. Khan et al. surveyed existing works on mobile phone sensing and proposed one of the first classifications on 


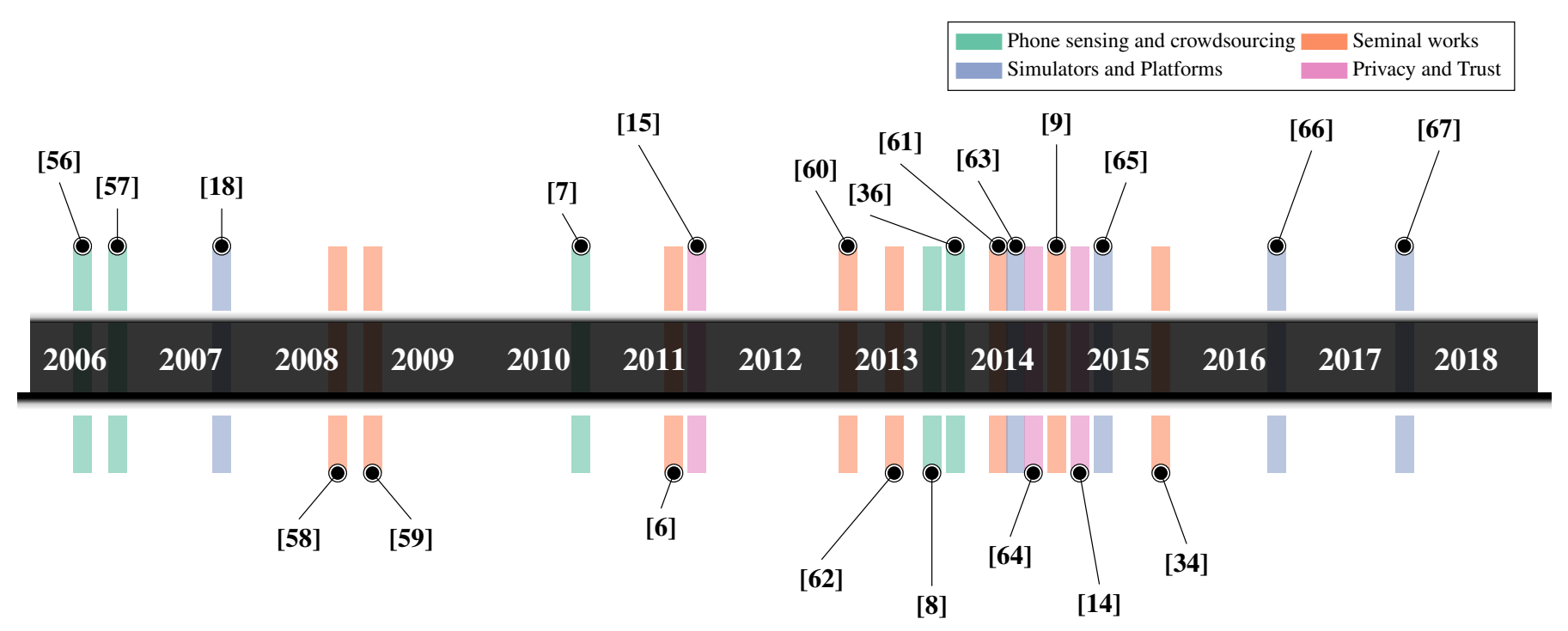

Fig. 3. MCS timeline. It presents the main works that have contributed to the evolution of MCS paradigm, divided between mobile phone sensing and crowdsourcing, seminal works, simulators and platforms, privacy and trust.

user involvement [9]. Vastardis et al. presented the existing architectures in mobile social networks, their social properties, and key research challenges [62]. MOSDEN was one among the first collaborative sensing frameworks operating with mobile phones to capture and share sensed data between multiple distributed applications and users [63].

2014. A team in the University of Bologna launches the ParticipAct Living Lab. The core lab activities lead to one of the first large-scale real-world experiments involving data collection from sensors of smartphones of 200 students for one year in the Emilia Romagna region of Italy [64]. Kantarci et al. propose a reputation-based scheme to ensure data trustworthiness, where IoT devices can enhance public safety managing crowdsensing services provided by mobile devices with embedded sensors [65]. The human factor as one of the most important components of MCS. Ma et al. investigate how human mobility correlates with sensing coverage and data transmission requirements [37]. Guo et. al define Mobile Crowdsensing in [10] by specifying the required features for a system to be defined as a MCS system. The article provides a retrospective study of MCS paradigm as an evolution of participatory sensing. Pournajaf et al. described the threats to users' privacy when personal information is disclosed and outlined how privacy mechanisms are utilized in existing sensing applications to protect the participants [15]. Tanas et al. were among the first to use ns-3 network simulator to assess the performance of crowdsensing networks. The work exploits specific features of ns-3, such as the mobility properties of network nodes combined with ad-hoc wireless interfaces [66].

2015. Guo et al. investigate the interaction between machine and human intelligence in crowdsensing processes, highlighting the fundamental role of having humans in the loop [35].

2016. Chessa et al. describe how to utilize socio-technical networking aspects to improve the performance of MCS

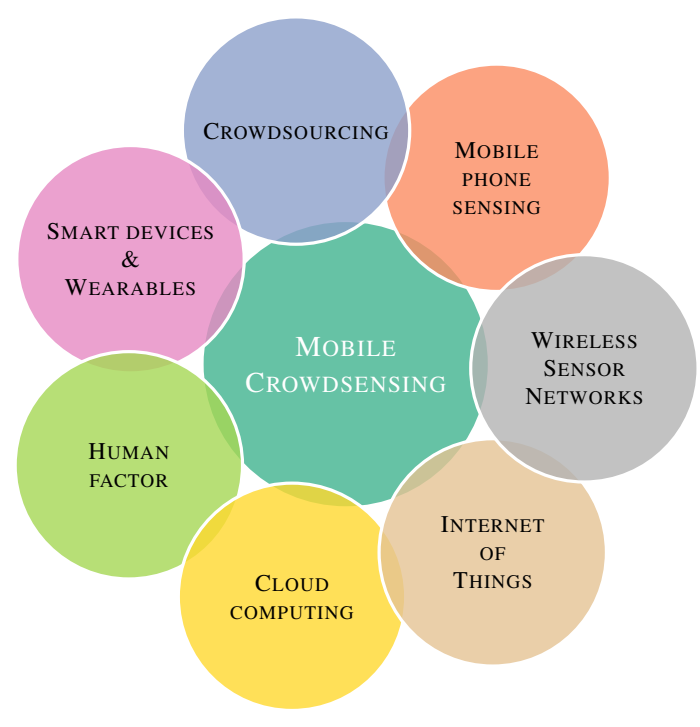

Fig. 4. Factors contributing to the rise of MCS

campaigns [67].

2017. Fiandrino et al. introduce CrowdSenSim, the first simulator for crowdsensing activities. It features independent modules to develop use mobility, location, communication technologies and sensors involved according to the specific sensing campaign [68].

\section{Factors Contributing to the Rise of MCS}

Several factors have contributed to the rise of MCS as one of the most promising data collection paradigm (see Fig. 4).

Mobile Phone Sensing. As mentioned above, mobile phone sensing is the forefather of MCS. Unlike MCS, the objective phone sensing applications are at the individual level. For 
example, mobile applications used for fitness utilize the GPS and other sensors (e.g., cardiometer) to monitor sessions [69]. Ubifit encourages users to monitor their daily physical activities such as running, walking or cycling [70]. Health care is another domain in which individual monitoring is fundamental, for diet [19] or detection and reaction to elderly people falls [71] [74]. Other examples consist of distinguishing transportation modes [75]-[78], recognition of speech [79] and driving style [80] and for indoor navigation [81]-[83].

Mobile smart devices/Wearables. The transition from mobile phones to smart mobile devices has boosted the rise of MCS. While mobile phones only allowed phone calls and text messages, smartphones are equipped with sensing, computing and communication capabilities. In addition to smartphones, tablets and wearables are other smart devices with the same properties. With wearables, user experience is fully included in the technology process. In [84], the authors present an application involving a WSN in a scenario focusing on sport and physical activities. Wearables and body sensor networks are fundamental in health-care monitoring including sports activities, medical treatments, and nutrition [85], [86]. In [87], the authors focus on inertial measurements units (IMUs) and wearable motion tracking systems for cost-effective motion tracking with a high impact in human-robot interaction.

Crowdsourcing. Crowdsourcing is the key that has steered phone sensing towards crowdsensing by enforcing communityoriented application purposes and leveraging large user participation for data collection. According to the discipline, several definitions of crowdsourcing have been proposed. An analysis of this can be found in [88]-[90]. Howe in [57] coined the original term. According to his definition, crowdsourcing represents the act of a company or an institution in outsourcing tasks formerly performed by employees to an undefined network of people in the form of an open call. This enforces peer-production when the job is accomplished collaboratively, but crowdsourcing is not necessarily only collaborative. A multitude of single individuals accepting the open call still matches with the definition. Incentives mechanisms have been proposed since the early days of crowdsourcing. Several studies consider Amazon's Mechanical Turk as one of the most important examples of incentive mechanisms for micro-tasks, the paradigm allowing to engage a multitude of users for short time and low monetary cost [91], [92].

Human factor. The fusion between complementary roles of human and machine intelligence builds on including humans in the loop of sensing, computing, and communicating processes [35]. The impact of the human factor is fundamental in MCS for several reasons. First of all, mobility and intelligence of human participants guarantee higher coverage and better context awareness if compared to traditional sensor networks. Also, users maintain by themselves the mobile devices and provide periodic recharge. Designing efficient human-in-theloop architecture is challenging. In [93], the authors analyze how to exploit the human factor, for example by steering behaviors after a learning phase. Learning and prediction typically require probabilistic methods [94].

Cloud computing. Considering the limited resources of local data storage in smart devices, processing such data usually takes place in the cloud [95], [96]. MCS is one of the most prominent applications in cloud-centric IoT systems, where smart devices offer resources (the embedded sensing capabilities) through cloud platforms on a pay-as-you-go basis [97]-[99]. Mobile devices contribute to a considerable amount of gathered information that needs to be stored for analysis and processing. The cloud enables to easily access shared resources and common infrastructure with a ubiquitous approach for efficient data management [100], [101].

Internet of Things (IoT). The IoT paradigm is characterized by a high heterogeneity of end systems, devices, and link layer technologies. Nonetheless, MCS focuses on urban applications, which narrows down the scope of applicability of IoT. To support the smart cities vision, urban IoT systems should improve the quality of citizens' life and provide added value to the community by exploiting the most advanced ICT systems [29]. In this context, MCS complements existing sensing infrastructures by including humans in the loop.

Wireless Sensor Networks (WSN). Wireless sensor networks are sensing infrastructures employed to monitor phenomena. In MCS, the sensing nodes are human mobile devices. As WSN nodes have become more powerful with time, multiple applications can run over the same WSN infrastructure trough virtualization [102]. WSNs face several scalability challenges. The Software-Defined Networking (SDN) approach can be employed to address these challenges and augment efficiency and sustainability under the umbrella of software-defined wireless sensor networks (SDWSN) [103]. The proliferation of small sensors has led to the production of massive amounts of data in smart communities, which cannot be effectively gathered and processed in WSNs due to their weak communication capability. Merging the concept of WSNs and cloud computing is a promising solution investigated in [104]. In this work, the authors introduce the concept of sensors clouds and classify the state of the art of WSNs by proposing a taxonomy on different aspects, such as communication technologies and type of data.

\section{Acronyms used in the MCS Domain}

MCS encompasses different fields of research and it exists different ways of offering MCS services (see Table II). This Subsection overviews and illustrates the most important methods.

$\mathbf{S}^{2}$ aaS. MCS follows a Sensing as a Service ( $\left.\mathrm{S}^{2} \mathrm{aaS}\right)$ business model, which makes data collected from sensors available to cloud users [98], [105], [111]. Consequently, companies and organizations have no longer the need to acquire infrastructure to perform a sensing campaign, but they can exploit existing ones on a pay-as-you-go basis. The efficiency of $\mathrm{S}^{2}$ aaS models is defined in terms of the revenues obtained and the costs. The organizer of a sensing campaign, such as a government agency, an academic institution or a business corporation, sustains costs to recruit and compensate users for their involvement [112]. The users sustain costs while contributing data too, i.e., the energy spent from the batteries for sensing and reporting data and, eventually, the data subscription plan if cellular connectivity is used for reporting. 
TABLE II

ACronyms RELATED to THE Mobile CrowdSENSING Domain

\begin{tabular}{|c|c|c|c|}
\hline ACRONYM & MEANING & DESCRIPTION & REFERENCES \\
\hline $\mathrm{S}^{2} \mathrm{aaS}$ & Sensing as a Service & Provision to the public of data collected from sensors & [98], [105] \\
\hline Senaas & Sensor as a Service & Exposure of IoT cloud's sensors capabilities and data in the form of services & [106] \\
\hline SAaaS & Sensing and Actuation as a Service & Provision of simultaneous sensing and actuation resources on demand & [107] \\
\hline MCSaaS & Mobile CrowdSensing as a Service & $\begin{array}{l}\text { Extension and adaptation of the SAaaS paradigm to deploy and enable rapidly } \\
\text { mobile applications, providing sensing and processing capabilities }\end{array}$ & [108] \\
\hline MaaS & Mobility as a Service & $\begin{array}{l}\text { Combination of options from different transport providers into a single mobile } \\
\text { service }\end{array}$ & [109] \\
\hline SIaaS & Sensing Instrument as a Service & $\begin{array}{l}\text { Provision virtualized sensing instruments capabilities and shares them as a } \\
\text { common resource }\end{array}$ & [99] \\
\hline MAaaS & Mobile Application as a Service & $\begin{array}{l}\text { Provision of resources for storage, processing and delivery of sensed data to } \\
\text { cloud applications }\end{array}$ & [95] \\
\hline MSaaS & Mobile Sensing as a Service & Sharing data collected from mobile devices to other users in the context of ITS & [110] \\
\hline
\end{tabular}

SenaaS. Sensor-as-a-Service has proposed to encapsulate both physical and virtual sensors into services according to Service Oriented Architecture (SOA) [106]. SenaaS mainly focuses on providing sensor management as a service rather than providing sensor data as a service. It consists of three layers: real-world access layer, semantic overlay layer, and services virtualization layer. the real-world access layer is responsible for communicating with sensor hardware. Semantic overlay layer adds a semantic annotation to the sensor configuration process. Services virtualization layer facilitates the users.

SAaaS. In SAaaS sensors and actuators guarantee tiered services through devices and sensor networks or other sensing platforms [107], [113]. The sensing infrastructure is characterized by virtual nodes, which are mobile devices owned by citizens that join voluntarily and leave unpredictably according to their needs. In this context, clients are not passive interfaces to Cloud services anymore, but they contribute through their communication, sensing, and computational capabilities.

MCSaaS. Virtualizing and customizing sensing resources, starting from the capabilities provided by the SAaaS model, allows for concurrent exploitation of pools of devices by several platform/application providers [108]. Delivering MCSaaS may further simplify the provisioning of sensing and processing activities within a device or across a pool of devices. Moreover, decoupling the MCS application from the infrastructure promotes the roles of a sensing infrastructure provider that enrolls and manages contributing nodes.

MaaS. Mobility as a Service fuses different types of travel modalities, e.g., combines options from different transport providers into a single mobile service to make easier planning and payments. MaaS is an alternative to own a personal vehicle and makes it possible to exploit the best option for each journey. The multi-modal approach is at the core of MaaS. A traveler can exploit a combination of public transport, bike sharing, car service or taxi for a single trip. Furthermore, MaaS can include value-added services like meal-delivery.

SIaaS. Sensing Instrument as a Service indicates the idea to exploit data collection instruments shared through cloud infrastructure [99]. It focuses on a common interface which offers the possibility to manage physical sensing instrument while exploiting all advantages of using cloud computing technology in storing and processing sensed data.

MAaaS. Mobile Application as a Service indicates a model to deliver data in which the cloud computing paradigm provides resources to store, and process sensed data, specifically focused on applications developed for mobile devices [114].

MSaaS. Mobile Sensing as a Service is a concept based on the idea that owners of mobile devices can join data collection activities and decide to share the sensing capabilities of their mobile devices as a service to other users [110]. Specifically, this approach refers to the interaction with Intelligent Transportation Systems (ITS) and relies on continuous sensing from connected cars. The MSaaS way of delivering MCS service can constitute an efficient and flexible solution to the problem of real-time traffic management and data collection on roads and related fields (e.g., road bumping, weather condition).

\section{Mobile Crowdsensing in A Nutshell}

This Section presents the main characteristic of MCS. First, we introduce MCS as a layered architecture, discussing each layer. Then, we divide MCS data collection frameworks between domain-specific and general-purpose and survey the main works. After that, theoretical works on operational research and optimization problems are presented and classified. Then, we present platforms, simulators, and dataset. Subsec. III-F closes the section by providing final remarks and outlines the new taxonomies that we propose for each layer. These are the main focus of the upcoming Sections.

\section{A. MCS as a Layered Architecture}

Similarly to [40], [42], we present a four-layered architecture to describe the MCS landscape, as shown in Fig. 1. Unlike the rationale in [40], [42], our proposal follows the direction of command and control. From top to bottom, the first layer is the Application layer, which involves user- and task-related aspects. The second layer is the Data layer, which concerns storage, analytics, and processing operations of the collected information. The third layer is the Communication layer that characterizes communication technologies and reporting methodologies. Finally, at the bottom of the layered architecture, there is the Sensing layer that comprises all the aspects 


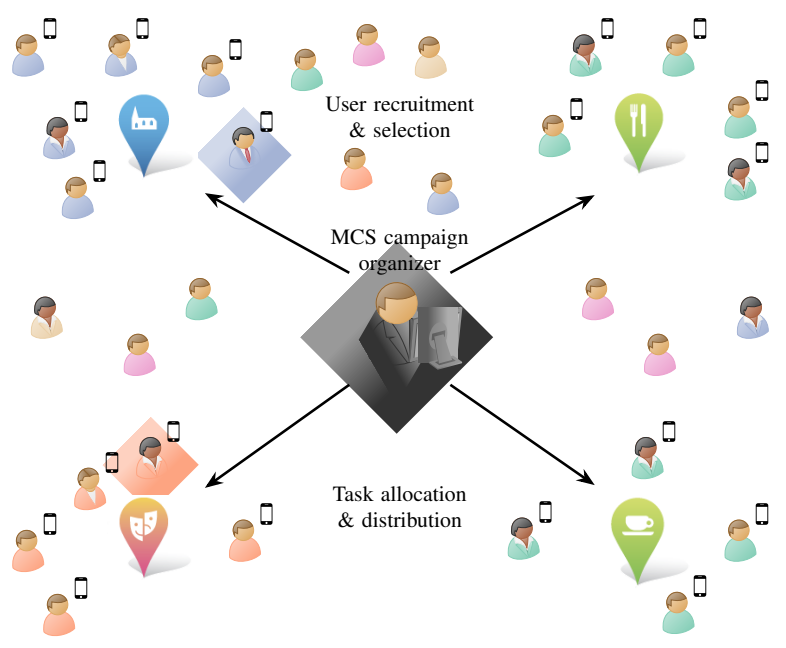

Fig. 5. Application layer. It comprises high-level task- and user-related aspects to design and organize a MCS campaign.

Data Analysis, Processing and Inference

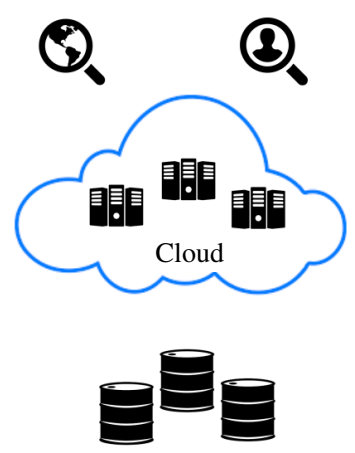

Raw Data

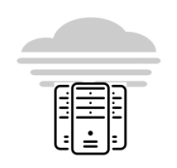

Fog

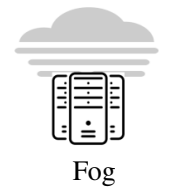

Fig. 6. Data layer. It includes technologies and methodologies to manage and process data collected from users.

involving the sensing process and modalities. In the following Sections, this architecture will be used to propose different taxonomies that will considered several aspects for each layer and according to classification to overview many MCS systems (Sec. IV, Sec. V, Sec. VI, and Sec. VII).

Application layer. Fig. 5 shows the application layer, which involves high-level aspects of MCS campaigns. Specifically, the phases of campaign design and organization, such as strategies for recruiting users and scheduling tasks and approaches related to task accomplishment, for instance, selection of user contributions to maximize the quality of information while minimizing the number of contributions. Sec. IV will present the taxonomies on the application layer and the related classifications.

Data layer. Fig. 6 shows the data layer, which comprises all components responsible to store, analyze, and process data received from contributors. It takes place in the cloud or can also be located closer to end users through fog servers, according to the need of the organizer of the campaign. For

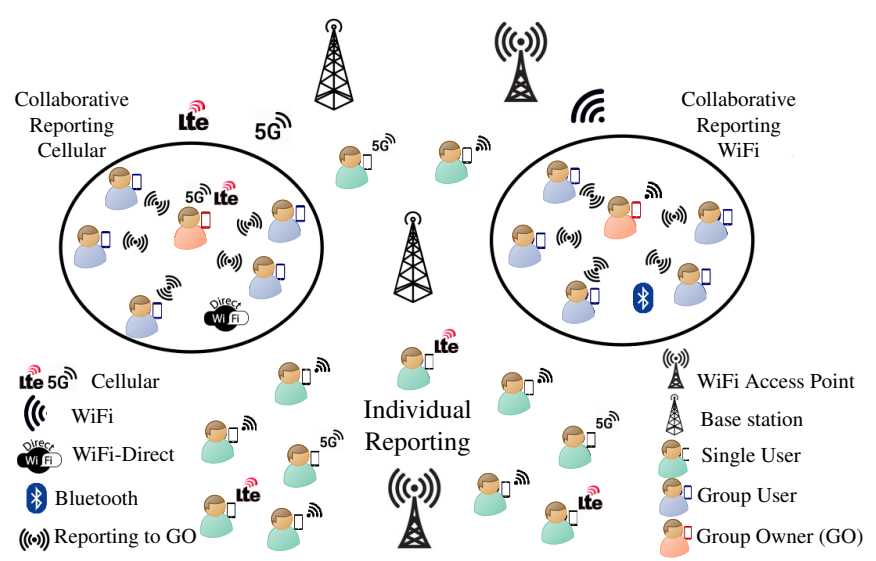

Fig. 7. Communication layer. It comprises communication technologies and data reporting typologies to deliver acquired data to the central collector.

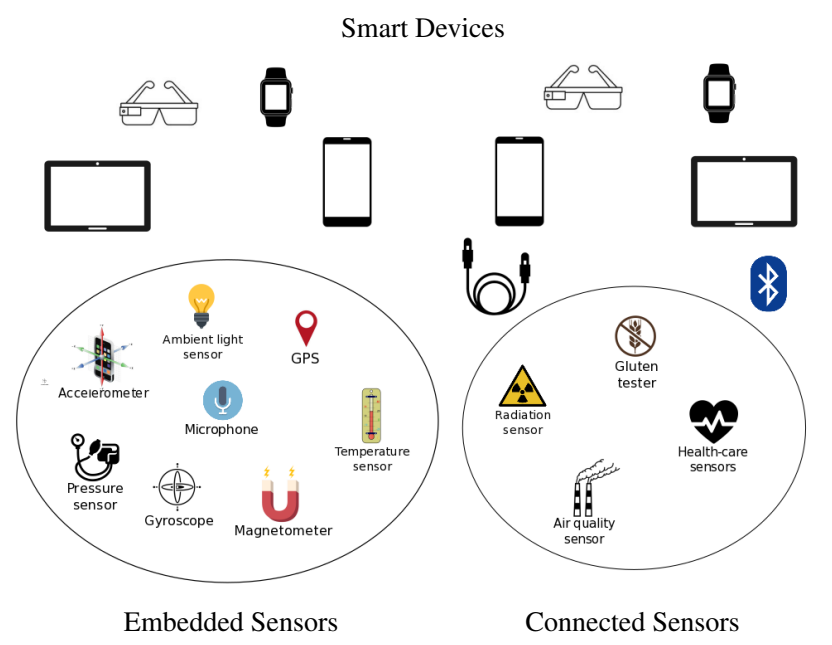

Fig. 8. Sensing layer. It includes the sensing elements needed to acquire data from mobile devices and sampling strategies to efficiently gather it.

instance, in this layer information is inferred from raw data and the collector computes the utility in receiving a certain type of data or the quality of acquired information. Taxonomies on data layer and related overview on works will be presented in Sec. V.

Communication layer. Fig. 7 shows the communication layer which includes both technologies and methodologies to deliver data acquired from mobile devices through their sensors to the cloud collector. The mobile devices are typically equipped with several radio interfaces, e.g., cellular, WiFi, Bluetooth, and several optimizations are possible to better exploit the communication interfaces, e.g., avoid transmission of duplicate sensing readings or coding redundant data. Sec. VI will propose taxonomies on this layer and classify works accordingly.

Sensing layer. Fig. 8 shows the sensing layer, which is at the heart of MCS as it describes sensors. Mobile devices usually acquire data through built-in sensors, but for specialized sensing campaigns other types of sensors can be connected. Sensors embedded in the device are fundamental for its 
normal utilization (e.g., accelerometer to automatically turn the orientation of the display or light sensor to regulate the brightness of the monitor), but can be exploited also to acquire data. They can include popular and widespread sensors such as gyroscope, GPS, camera, microphone, temperature, pressure, but also the latest generation sensors, such as NFC. Specialized sensors can also be connected to mobile devices via cable or wireless communication technologies (e.g., WiFi direct or Bluetooth), such as radiation, gluten, and air quality sensors. Data acquired through sensors is transmitted to the MCS platform exploiting the communication capabilities of mobile devices, as explained in the following communication layer. Sec. VII will illustrate taxonomies proposed for this layer and consequent classification of papers.

\section{B. Data Collection Frameworks}

The successful accomplishment of a MCS campaign requires to define with precision a number of steps. These depend on the specific MCS campaign purpose and range from the data acquisition process (e.g., deciding which sensors are needed) to the data delivery to the cloud collector (e.g., which communication technologies should be employed). The entire process is known as data collection framework (DCF).

DCFs can be classified according to their scope in domainspecific and general-purpose. A domain-specific DCF is specifically designed to monitor certain phenomena and provides solutions for a specific application. Typical examples are air quality [21], noise pollution monitoring [115] and also health care, such as the gluten sensor [116] for celiacs. Viceversa, General-purpose DCFs are not developed for a specific application but aim at supporting many applications at the same time.

Domain-specific DCFs. Domain-specific DCFs are specifically designed to operate for a given application, such as health care, environmental monitoring, and intelligent transportation among the others. One of the most challenging issues in smart cities is to create a seamless interconnection of sensing devices, communication capabilities, and data processing resources to provide efficient and reliable services in many different application domains [170]. Different domains of applicability typically correspond to specific properties and design requirements for the campaign, e.g., exploited sensors or type of data delivery. For instance, a noise monitoring application will use microphone and GPS and, usually, is delay-tolerant, while an emergency response application will typically require more sensors and real-time data reporting. In other words, domain-specific DCFs can be seen as use cases of MCS systems. The following Subsection presents the most promising application domains where MCS can operate to improve the citizens' quality of life in a smart city domain and overview existing works, as shown in Table III.

Emergency management and prevention. This category comprises all application related to monitoring and emergency response in case of accidents and natural disasters, such as flooding [24], earthquakes [117]-[119], fires, and nuclear disasters [120]. For instance, Creekwatch is an application developed by the IBM Almaden research center [24]. It allows to monitor the watershed conditions through crowdsensed data that consists of the estimation of the amount of water in the river bed, the amount of trash in the river bank, the flow rate, and a picture of the waterway.

Environmental Monitoring. Environmental sensing is fundamental for sustainable development of urban spaces and to improve citizens' quality of life. It aims to monitor the use of resources, the current status of infrastructures and urban phenomena that are well-known problems affecting the everyday life of citizens, e.g., air pollution is responsible for a variety of respiratory diseases and can be a cause of cancer if individuals are exposed for long periods. To illustrate with some examples, the Personal Environment Impact Report (PEIR) exploits location data sampled from everyday mobile phones to analyze on a per-user basis how transportation choices simultaneously impact on the environment and calculate the risk-exposure and impact for the individual [121]. Ear-Phone is an end-to-end urban noise mapping system that leverages compressive sensing to solve the problem of recovering the noise map from incomplete and random samples [122]. This platform is delay tolerant and exploits only WiFi because it aims at creating maps and does not need urgent data reporting. NoiseTube is a noise monitoring platform that exploits GPS and microphone to measure the personal exposure of citizen to noise in everyday life [123]. NoiseMap measures value in $\mathrm{dB}$ corresponding to the level of noise in a given location detected via GPS [115] In indoor environments, users can tag a particular type of noise and label a location to create maps of noise pollution in cities by exploiting WiFi localization [124]. In [125], the authors propose a platform that achieves tasks of environmental monitoring for smart cities with a fine granularity, focusing on data heterogeneity. Nowadays air pollution is a significant issue and exploiting mobility of humans to monitor air quality with sensors connected to their devices may represent a winwin solution with higher accuracy than fixed sensor networks. HazeWatch is an application developed in Sydney that relies on active citizen participation to monitor air pollution and is currently employed by the National Environment Agency of Singapore on a daily basis [22]. GasMobile is a small and portable measurement system based on off-the-shelf components, which aims to create air pollution maps [21] CommonSense allows citizens to measure their exposure at the air pollution and in forming groups to aggregate the individual measurements [56]. Counter-Strike consists of an iterative algorithm for identifying low-level radiation sources in the urban environment, which is hugely important for the security protection of modern cities [126]. Their work aim at making robust and reliable the possible inaccurate contribution of users. In [127], the authors exploit the flash and the camera of the smartphone as a light source and receptor. In collaboration with a dedicated sensor, they aim at measuring the amount of dust in the air. Recent discoveries of signature in the ionosphere related to earthquakes and tsunamis suggests that ionosphere may be used as a sensor that reveals Earth and space phenomena [128]. The Mahali Project utilizes GPS signals that penetrate the ionosphere to enable a tomographic analysis of the ionosphere, exploited as a global earth system sensor [129]. 
TABLE III

DOMAIN-SPECIFIC DATA COLLECTION FRAMEWORKS (DCFs)

\begin{tabular}{|c|c|c|}
\hline DOMAIN OF INTEREST & DESCRIPTION & REFERENCES \\
\hline Emergency prevention and management & $\begin{array}{l}\text { Prevention of emergencies (e.g., monitoring the amount of water in the river bed) } \\
\text { and post-disaster management (earthquakes or flooding) }\end{array}$ & [24], [117]-[120] \\
\hline Environmental monitoring & $\begin{array}{l}\text { Monitoring of resources and environmental conditions, such as air and noise } \\
\text { pollution, radiation }\end{array}$ & [21], [22], [56], [115], [121]-[129] \\
\hline E-commerce & $\begin{array}{l}\text { Collection, sharing and live-comparison of prices of goods from real stores or } \\
\text { specific places, such as gas stations }\end{array}$ & [130]-[133] \\
\hline Health care \& wellbeing & $\begin{array}{l}\text { Sharing of users' physical or mental conditions for remote feedback or exchange } \\
\text { of information about wellbeing like diets and fitness }\end{array}$ & [17], [19], [116], [134]-[136] \\
\hline Indoor localization & $\begin{array}{l}\text { Enabling indoor localization and navigation by means of MCS systems in GPS- } \\
\text { denied environments }\end{array}$ & [137]-[139] \\
\hline Intelligent transportation systems & $\begin{array}{l}\text { Monitoring of citizen mobility, public transport and services in cities, e.g., traffic } \\
\text { and road condition, available parking spots, bus arrival time }\end{array}$ & {$[20],[140]-[145]$} \\
\hline Mobile social networks & $\begin{array}{l}\text { Establishment of social relations, meeting, sharing experiences and data (photo and } \\
\text { video) of users with similar interests }\end{array}$ & {$[62],[146]-[155]$} \\
\hline Public safety & $\begin{array}{l}\text { Citizens can check, share and evaluate the level of crimes for each areas in urban } \\
\text { environments }\end{array}$ & [156], [157] \\
\hline Unmanned vehicles & $\begin{array}{l}\text { Interaction between mobile users and driver-less vehicles (e.g., aerial vehicles or } \\
\text { cars), which require high-precision sensors }\end{array}$ & [158]-[160] \\
\hline Urban planning & $\begin{array}{l}\text { Improving experience-based decisions on urbanization issues, such as street networks } \\
\text { design and infrastructure maintenance }\end{array}$ & [30], [161], [162] \\
\hline Waste management & $\begin{array}{l}\text { Citizens help to monitor and support waste-recycling operations, e.g., checking the } \\
\text { amount of trash or informing on dynamic waste collection routing }\end{array}$ & [25], [26] \\
\hline WiFi characterization & $\begin{array}{l}\text { Mapping of WiFi coverage with different MCS techniques, such as exploiting } \\
\text { passive interference power, measuring spectrum and received power intensity }\end{array}$ & {$[163]-[165]$} \\
\hline Others & $\begin{array}{l}\text { Specific domain of interest not included in the previous list, such as recommending } \\
\text { travel packages, detecting activity from sound patterns }\end{array}$ & [147], [148], [166]-[169] \\
\hline
\end{tabular}

E-commerce. Websites comparing prices of goods and services guide customer decisions and improve competitiveness, but they are typically limited to the online world where most of the information is accessible over the Internet. MCS can fill the gap connecting physical and digital worlds and allowing customers to report information and compare pricing in different shops or from different vendors [130]. To give a few examples, camera and GPS used in combination may track and compare fuel prices between different gas stations [131]. While approaching gas stations, an algorithm extracts the price from pictures recorded through the camera and associates it with the gas station exploiting the GPS. Collected information is compared with the one gathered by other users to detect most convenient petrol stations. In [130], the authors present a participatory sensing paradigm which can be employed to track price dispersion of similar consumer goods even in offline markets. Mobishop is a distributed computing system designed to collect, process, and deliver product price information from street-side shops to potential buyers, on their mobile phones, through receipt scanning [132]. LiveCompare represents another example of MCS solution that uses the camera to take pictures of bar codes and GPS for the market location to compare prices of goods in real-time [133].

Health Care and wellbeing. Health care allows diagnosing, treating and preventing illness, diseases, and injuries. It includes a broad umbrella of fields connected to health that ranges from self-diagnostics to physical activities, passing through diet monitoring. HealthAware is a system that uses the accelerometer to monitor daily physical activity and camera to take pictures of food that can be shared [17]. SPA is a smartphone assisted chronic illness self-management system that facilitates patient involvement exploiting regular feedbacks of relevant health data [134]. Yi et al. propose an Android- based system that collects, displays, and sends acquired data to the central collector [135]. Dietsense automatically captures pictures of food, GPS location, timestamp references, and a microphone detects in which environment the sample was collected for dietary choices [19]. It aims to share data to the crowd and allows just-in-time annotation, which consists of reviews captured by other participants. AndWellness is a personal data collection system that exploits the smartphone's sensors to collect and analyze data from user experiences [136].

Indoor localization. Indoor localization refers to the process of providing localization and navigation services to users in indoor environments. In such a scenario, the GPS does not work, and other sensors are employed. Fingerprinting is a popular technique for localization and operates by constructing a location-dependent fingerprint map. MobiBee collects fingerprints by exploiting quick response $(\mathrm{QR})$ codes, e.g., posted on walls or pillars as location tags [137]. A location marker is designed to guarantee that the fingerprints are collected at the targeted locations. WiFi fingerprinting presents some drawbacks, such as a very labor-intensive and time-consuming radio map construction, and the Received Signal Strength (RSS) variance problem, which is caused by the heterogeneity of devices and environmental context instability. In particular, RSS variance severely degrades the localization accuracy. RCILS is a robust crowdsourcing indoor localization system which aims at constructing the radio map with smartphone's sensors [138]. RCILS abstracts the indoor map as a semantics graph in which the edges are the possible user paths and the vertexes are the location where users perform special activities. Fraud attacks frequently compromise reference tags employed for getting location annotations. Three types of location-related attacks in indoor MCS are considered in [139], including tag forgery, tag misplacement, and tag removal. To deal with these attacks, 
the authors propose location-dependent fingerprints as supplementary information for improving location identification, a truth discovery algorithm to detect falsified data, and visiting patterns to reveal tag misplacement and removal.

Intelligent Transportation Systems. MCS can also support ITS to provide innovative services, improve cost-effectiveness and efficiency of transportation and traffic management systems [140]. It includes all the applications related to traffic, vehicles, public transports, and road conditions. In [141], social networks are exploited to acquire direct feedback and potentially valuable information from people to acquire awareness in ITS. In particular, it verifies the reliability of pollution-related social networks feedbacks into ITS systems. In [142], the authors present a system that predicts bus arrival times and relies on bus passengers' participatory sensing. The proposed model is based only on the collaborative effort of the participants and it uses cell tower signals to locate the users, preventing them from battery consumption. Furthermore, it uses accelerometer and microphone to detect when a user is on a bus. Wind warning systems alert drivers while approaching bridges in case of dangerous wind conditions. WreckWatch is a formal model that automatically detects traffic accidents using the accelerometer and acoustic data, immediately send a notification to a central emergency dispatch server and provide photographs, GPS coordinates, and data recordings of the situation [143]. Nericell is a system used to monitor road and traffic conditions, which uses different sensors for rich sensing and detects potholes, bumps, braking, and honking [20]. It exploits the piggybacking mechanisms on users' smartphones to conduct experiments on the roads of Bangalore. Safestreet detects and reports the potholes and surface abnormalities of roads exploiting patterns from accelerometer and GPS [144]. The evaluation exploits datasets collected from thousands of kilometers of taxi drives in Mumbai and Boston. VTrack is a system which estimates travel time challenging with energy consumption and inaccurate position samples [145]. It exploits a HMM (Hidden Markov Model)-based map matching scheme and travel time estimation method that interpolates sparse data to identify the most probable road segments driven by the user and to attribute travel time to those segments.

Mobile Social Networks (MSNs). MSNs are communication systems that allow people with similar interests to establish social relations, meet, converse and share exploiting mobile devices [62], [146]. In this category, the most fundamental aspect is the collaboration between users who share the data sensed through smartphone sensors, such as recognized activities and locations. Crowdsense@place is a system that focuses on scaling properties of place-centric crowdsensing to provide place related informations [147], including the relationship between users and coverage [148]. MSNs focus not only on the behavior but also on the social needs of the users [62]. The CenceMe application is a system which combines the possibility to use mobile phone embedded sensors for the sharing of sensed and personal information through social networking applications [59]. It takes a user status in terms of his activity, context or habits and shares them in social networks. Micro-Blog is a location-based system to automatically propose and compare the context of the users by leveraging new kinds of application-driven challenges [60]. EmotionSense is a platform for social psychological studies based on smartphones [149]: its key idea is to map not only activities, but also emotions, and to understand the correlation between them. It gathers user emotions as well as proximity and patterns of conversations by processing the audio from the microphone. MobiClique is a system which leverages already existing social networks and opportunistic contacts between mobile devices to create ad hoc communities for social networking and social graph based opportunistic communications [150]. MIT Serendipity is one of the first projects that explored the MSN aspects [151]: it automates informal interactions using Bluetooth. SociableSense is a platform that realizes an adaptive sampling scheme based on learning methods and a dynamic computation distribution mechanism based on decision theory [152]. This system captures user behavior in office environments and provides the participants with a quantitative measure of sociability of them and their colleagues. WhozThat is a system built on opportunistic connectivity for offering an entire ecosystem on which increasingly complex context-aware applications can be built [153]. MoVi, a Mobile phone-based Video highlights system, is a collaborative information distillation tool capable of filtering events of social relevance. It consists of a trigger detection module that analyses the sensed data of several social groups and recognizes potentially interesting events [154]. Darwin phones is a work that combines collaborative sensing and machine learning techniques to correlate human behavior and context on smartphones [155]. Darwin is a collaborative framework based on classifier evolution, model pooling and collaborative sensing, which aims to infer particular moments of people's life.

Public Safety. Nowadays public safety is one of the most critical and challenging issues for the society, which includes protection and prevention from damages, injuries or generic dangers including burglary, trespassing, harassment, and inappropriate social behaviors. iSafe is a system for evaluating the safety of citizens exploiting the correlation between information obtained from geo-social networks with crime and census data from Miami county [156]. In [157], the authors investigate how public safety could leverage better commercial IoT capabilities to deliver higher survivability to the warfighter or first responders while reducing costs and increasing operational efficiency and effectiveness. This paper reviews the main tactical requirements and the architecture, examining gaps and shortcomings in existing IoT systems across the military field and mission-critical scenarios.

Unmanned Vehicles Recently, unmanned vehicles have become popular and MCS can play an important role in this field. Indeed, both unmanned aerial vehicles (UAVs) and driver-less cars are equipped with different types of high-precision sensors and frequently interact with mobile devices and users [158]. In [159], the authors investigate the problem of energy-efficient joint route planning and task allocation for fixed-wing UAVaided MCS system. The corresponding joint optimization problem is developed as a two-stage matching problem. In 
the first stage, genetic algorithms are employed to solve the route planning. In the second stage, the proposed Gale-Shapley algorithm solves the task assignment. To provide a global view of traffic conditions in urban environments, [160] proposes a trust-aware MCS technique based on UAVs. The system receives real traffic information as input and UAVs build the distribution of vehicles and RSUs in the network.

Urban planning Urban planning in the context of MCS is related to improve experience-based decisions on urbanization issues by analyzing data acquired from different devices of citizens in urban environments. It aims to improve citizens' quality of life by exploiting sensing technologies, data analytics, and processing tools to deploy infrastructures and solutions [161]. For instance, some studies investigate the impact of the street networks on the spatial distribution of urban crimes, predicting that violence happens more often on well-connected roads [162]. In [30], the authors show that data acquired from accelerometers embedded in smartphones of car drivers can be used for monitoring bridge vibrations by detecting several modal frequencies.

Waste Management. Although apparently related to environmental monitoring, we classify waste management in a standalone category on purpose. Waste management is an emerging field which aims to handle effectively waste collection, i.e., how to appropriately choose location of bins and optimal routes of collecting trucks, recycling, and all the related processes [171]. WasteApp presents a design and an implementation of a mobile app [26]. It aims at merging behavioral studies and standard features of existing mobile apps with a co-design methodology that gathers real user needs for waste recycling. Garbage Watch employs citizens to monitor the content of recycling bins to enhance recycling program [25].

WiFi characterization. This application characterizes the $\mathrm{WiFi}$ coverage in a certain area, for example by measuring spectrum and interference [164]. In [163], the authors propose a model for sensing the volume of wireless activity at any frequency exploiting the passive interference power. This technique utilizes a non-intrusive way of inferring the level of wireless traffic, without extracting data from devices. Furthermore, the presented approach is independent of the traffic pattern and requires only approximate location information. MCNet enables WiFi performance measurements and mapping data about WLAN taken from users that participate in the sensing process [165].

Others. This category includes works not classified in the previous fields of application, but still focusing on specific domains. SoundSense is a scalable framework for modeling sound events on smartphones, using a combination of supervised and unsupervised learning techniques to classify both general sound types and discover sound events specific to individual users [166]. ConferenceSense acquires data, extract, and understand community properties to sense large events like conferences [167]. It uses some sensors and user inputs to infer contexts such as the beginning and the end of a group activity. In [169], the authors propose travel packages exploiting a recommendation system to help users in planning travels by leveraging data collected from crowdsensing. They distinguish user preferences, extract points of interest (POI) and determine location correlations from data collected. Them personalized travel packages are determined by considering personal preferences, POI, temporal and spatial correlations. Improve the Location Reliability (ILR) is a scheme in which participatory sensing is used to achieve data reliability [168]. The key idea of this system is to validate locations using photo tasks and expanding the trust to nearby data points using periodic Bluetooth scanning. The participants send photo tasks from the known location, which are manually or automatically validated.

General-purpose DCFs. General-purpose DCFs investigate common issues in MCS systems, independently from their domains of interest. For instance, energy efficiency and task coverage are two fundamental factors to investigate, independently if the purpose of a campaign is environmental monitoring, health care or public safety. This Subsection presents the main aspects of general-purpose DCFs and classifies existing works in the domain (see Table IV for more insights).

Context awareness. Understanding mobile device context is fundamental for providing higher data accuracy and does not waste battery of smartphones when sensing does not meet the application requirements (e.g., taking pictures when a mobile device is in a pocket). Here-n-now is a work that investigates the context-awareness, combining data mining and mobile activity recognition [172]. Lifemap provides location-based services, exploiting inertial sensors to provide also indoor location information [173]. Gathered data combines GPS and WiFi positioning systems to detect context awareness better. To achieve a wide acceptance of task execution, it is fundamental a deep understanding of factors influencing user interaction patterns with mobile apps. Indeed, they can lead to more acceptable applications that can report collected data at the right time. In [194], the authors investigate the interaction behavior with notifications concerning the activity of users and their location. Interestingly, their results show that user willingness to accept notifications is strongly dependent on location but only with minor relation to their activities.

Energy efficiency. To foster large participation of users, it is necessary that sensing and reporting operations do not drain their batteries. Consequently, energy efficiency is one of the most important keys to the success of a campaign. Piggyback Crowdsensing [174] aims to lower the energy overhead of mobile devices exploiting the opportunities in which users place phone calls or utilize applications. Devices do not need to wake up from an idle state to specifically report data of the sensing campaign, saving energy. Other DCFs exploit feedback from the collector to avoid useless sensing and reporting operations. In [175], a deterministic distributed DCF aims to foster energyefficient data acquisition in cloud-based MCS systems. The objective is to maximize the utility of the central collector in acquiring data in a specific area of interest from a set of sensors while minimizing the battery costs users sustain to contribute information. A distributed probabilistic algorithm is presented in [176], where a probabilistic design regulates the amount of data contributed from users in a certain region of interest to minimize data redundancy and energy waste. The algorithm is 
TABLE IV

General-purpose Data Collection Frameworks (DCFs)

\begin{tabular}{llc}
\hline TARGET & DESCRIPTION & REFERENCES \\
\hline Context awareness & $\begin{array}{l}\text { Combination of data mining and activity recognition techniques for context } \\
\text { detection }\end{array}$ & {$[172],[173]$} \\
Energy efficiency & $\begin{array}{l}\text { Strategies to lower the battery drain of mobile devices during data sensing and } \\
\text { reporting }\end{array}$ & {$[174]-[178]$} \\
Resource allocation & $\begin{array}{l}\text { Strategies for efficient resource allocation during data contribution, such as } \\
\text { channel condition, power spectrum, computational capabilities }\end{array}$ & {$[179]-[181]$} \\
Scalability & $\begin{array}{l}\text { Solutions to develop DCFs with good scalability properties during run-time data } \\
\text { acquisition and processing } \\
\text { Sefinition of requirements for task accomplishment, such as spatial and temporal } \\
\text { coverage }\end{array}$ & {$[182],[183]$} \\
Trustworthiness and privacy & $\begin{array}{l}\text { Strategies to address issues related to preserve privacy of the contributing users } \\
\text { and integrity of reported data }\end{array}$ & {$[188]-[193]$} \\
\hline
\end{tabular}

based on limited feedback from the central collector and does not require users to complete a specific task. EEMC (Enabling energy-efficient mobile crowdsensing) aims to reduce energy consumption due to data contribution both for individuals and the crowd while making secure the gathered information from a minimum number of users within a specific timeframe [177]. The proposed framework includes a two-step task assignment algorithm that avoids redundant task assignment to reduce the average energy consumption. In [178], the authors provide a comprehensive review of energy saving techniques in MCS and identify future research opportunities. Specifically, they analyze the main causes of energy consumption in MCS and present a general energy saving framework named ESCrowd, which aims to describe the different detailed MCS energy saving techniques.

Resource allocation. Meeting the demands of MCS campaigns requires to allocate resources efficiently. The predictive Channel-Aware Transmission (pCAT) scheme is based on the fact that much less spectrum resource allocation is required when the channel is in good condition [179]. Considering user trajectories and recurrent spots with a good channel condition, the authors suggest that background communication traffic can be scheduled by the client according to application data priority and expected quality data. In [180], the authors consider precedence constraints in specialized tasks among multiple steps, which require flexibility in order to the varying level of their dependency. Furthermore, they consider variations of loads needed for accomplishing different tasks and require allocation schemes that are simple, fast, decentralized and provide the possibility to choose contributing users. The main contribution of authors is to focus on the performance limits of MCS systems regarding these issues and propose efficient allocation schemes, claiming they meet the performance under the considered constraints. SenSquare is a MCS architecture that aims to save resources for both contributors and stakeholders, reducing the amount of data to deliver while maintaining its precision and rewarding users for their contribution [181]. The authors developed a mobile application as a case study to prove the effectiveness of SenSquare by evaluating the precision of measures and resources savings.
Scalability. MCS systems require large participation of users to be effective and make them scalable to large urban environments with thousands of citizens is paramount. In [182], the authors investigate the scalability of data collection and run-time processing with an approach of mobile/onboard data stream mining. This framework provides efficiency in energy and bandwidth with no consistent loss of information that mobile data stream mining could obtain. In [183], the authors investigate signicant issues in MCS campaigns, such as heterogeneity of mobile devices and propose an architecture to lower the barriers of scalability exploiting VM-based cloudlets.

Sensing task coverage. To accomplish a task, a sensing campaign organizer requires users to effectively meet application demands, including space and time coverage. Some DCFs address the problems related to spatio-temporal task coverage. A solution to create high-accurate monitoring is to design an online scheduling approach that considers the location of devices and sensing capabilities to select contributing nodes, developing a multi-sensor platform [184]. STREET is a DCF that investigates the problem of the spatial coverage, classifying it between partial coverage, full coverage or k-coverage. It aims to improve the task coverage in an energy-efficient way by requiring updates only when needed [185]. Another approach to investigate the coverage of a task is to estimate the optimal number of citizens needed to meet the sensing task demands in a region of interest over a certain period of time [186]. Sparse MCS aims to lower costs (e.g., user rewarding and energy consumption) while ensuring data quality by reducing the required number of sensing tasks allocated [187]. To this end, it leverages the spatial and temporal correlation among the data sensed in different sub-areas.

Trustworthiness and Privacy. As discussed, a large participation of users is essential to make effective a crowdsensing campaign. To this end, one of the most important factors for lowering barriers of citizens unwillingness in joining a campaign is to guarantee their privacy. On the other hand, an organizer needs to trust participants and be sure that no malicious nodes contribute unreliable data. In other words, MCS systems require mechanisms to guarantee privacy and trustworthiness to all components. There exist a difference between the terms 
trustworthiness and truthfulness when it comes to MCS. The former indicates how valuable and trusted is data contributed from users. The latter indicates how truthful a user is when joining the auction, because a user may want to increase the data utility to manipulate the contribution.

In MCS systems, the problem of ensuring trustworthiness and privacy remains open. First, data may be often unreliable, due to low-quality sensors, heterogeneous sources, noise, and other factors. Second, data may reveal sensitive information, such as pictures, mobility patterns, user behavior, and individual health. Deco is a DCF that investigates malicious and erroneous contributions, which inevitably result in poor data quality [189]. It aims to detect and correct false and missing data by applying spatio-temporal compressive sensing techniques. In [188], the authors propose a framework whose objective is twofold. First, to extract reliable information from heterogeneous noisy and biased data collected from different devices. Then, to preserve users' privacy. To reach these goals, they design a non-interactive privacy-preserving truthful-discovery system that follows a two-server model and leverages Yao's Garbled circuit to address the problem optimization. BUR is a Basic User Recruitment protocol that aims to address privacy issues by preserving user recruitment [190]. It is based on a greedy strategy and applies secret sharing techniques to propose a secure user recruitment protocol. In [191], the authors investigate privacy concerns related to incentive mechanisms, focusing on Sybil attack where a user may illegitimately pretend multiple identities to receive benefits. They design a Sybil-proof incentive mechanism in online scenarios that depends on users' flexibility in performing tasks and present single-minded and multi-minded cases. DTRPP is a Dynamic Trust Relationships aware data Privacy Protection mechanism that combines key distribution with trust management [192]. It is based on a dynamic management of nodes and estimation of the trust degree of the public key, which is provided by encountering nodes. QnQ is a Quality and Quantity based unified approach that proposes an event-trust and user-reputation model to classify users according to different classes, such as honest, selfish, or malicious [193]. Specifically, QnQ is based on a rating feedback scheme that evaluates the expected truthfulness of specific events by exploiting a QoI metric to lower effects of selfish and malicious behaviors.

\section{Theoretical Works, Operational Research and Optimization}

This Subsection presents analytical and theoretical studies proposed in the domain of MCS, such as operational research and optimization problems. While the previous Subsection discusses the technicalities of data collection, this Subsection presents research works that analyze MCS systems from a theoretical perspective. We classify these works according to their target (see Table V).

We remark that this Subsection differentiates from the previous ones because it focuses on aspects like abstract problem formulation, key challenges, and proposed solutions. For instance, while the previous Section presents data collection frameworks that acquire data leveraging context awareness, this Section presents a paragraph on context awareness that investigates how to formulate the problem and the proposed optimized solutions without practical implementation.

Trade-off between amount and quality data with energy consumption. In the last years, many researchers have focused their attention on the trade-off between the amount and quality of collected data and the corresponding energy consumption. The desired objectives are:

- to obtain high quality of contributed data (i.e., to maximize the utility of sensing) with low energy consumption;

- to limit the collection of low-quality data.

The Minimum Energy Single-sensor task Scheduling (MESS) problem [196] analyzes how to optimally schedule multiple sensing tasks to the same user by guaranteeing the quality of sensed data while minimizing the associated energy consumption. The problem upgrades to be Minimum Energy Multi-sensor task Scheduling (MEMS) when sensing tasks require the use of multiple sensors simultaneously. In [195], the authors investigate task allocation and propose a first-infirst-out (FIFO) task model and an arbitrary deadline (AD) task model for offline and online settings. The latter scenario is more complex because the requests arrive dynamically without prior information. The problem of ensuring energy-efficiency while minimizing the maximum aggregate sensing time is NPhard even when tasks are defined a priori [197]. The authors first investigate the offline allocation model and propose an efficient polynomial-time approximation algorithm with a factor of $2-1 / m$, where $m$ is the number of mobile devices joining the system. Then, focusing on the online allocation model, they design a greedy algorithm which achieves a ratio of at most $m$. In [36], the authors present a distributed algorithm for maximizing the utility of sensing data collection when the smartphone cost is constrained. The design of the algorithm considers a stochastic network optimization technique and distributed correlated scheduling.

Sensing Coverage. The space and temporal demands of a MCS campaign define how sensing tasks are generated. Following the definition by $\mathrm{He}$ et al. [241], the spatial coverage is defined as the total number of different areas covered with a given accuracy, while the temporal coverage is the minimum amount of time required to cover all regions of the area.

By being budget-constrained, [198] investigates how to maximize the sensing coverage in regions of interest and proposes an algorithm for sensing task allocation. The article also shows considerations on budget feasibility in designing incentive mechanisms based on a scheme which determines proportional share rule compensation. To maximize the total collected data being budged-constrained, in [203] organizers pay participants and schedule their sensing time based on their bids. This problem is NP-hard and the authors propose a polynomial-time approximation.

The effective target coverage measures the capability to provide sensing coverage with a certain quality to monitor a set of targets in an area [199]. The problem is tackled with queuing model-based algorithm where the sensors arrive and leave the coverage region following a birth process and death process. The $\alpha, T$-coverage problem [201] consists in ensuring that each point of interest in an area is sensed by at least one 
TABLE V

THEORETICAL WORKS ON OPERATIONAL RESEARCH AND OPTIMIZATION PROBLEMS

\begin{tabular}{llll}
\hline TARGET & OBJECTIVE & REFERENCES \\
\hline Trade-off data vs. energy & $\begin{array}{l}\text { Maximization of the amount and quality of gathered data while minimizing the } \\
\text { energy consumption of devices }\end{array}$ & $\begin{array}{l}\text { Focus on how to efficiently address the requirements on task sensing coverage } \\
\text { in space and temporal domains }\end{array}$ \\
Sensing coverage & $\begin{array}{l}\text { Efficient task allocation among participants leveraging diverse techniques and } \\
\text { approaches }\end{array}$ & $\begin{array}{l}\text { Efficient user recruitment to meet the requirements of a sensing campaign while } \\
\text { minimizing the cost }\end{array}$ & [198]-[205] \\
Task allocation & $\begin{array}{l}\text { It consists in exploiting context-aware sensing to improve system performance } \\
\text { in terms of delay, bandwidth, and energy efficiency }\end{array}$ & [206]-[217] \\
Maximization of task accomplishment under budget constraints or minimization & [112], [236]-[239], [240] \\
of budget to fully accomplish a task & [218]-[227] \\
\hline Budget-constrained & &
\end{tabular}

node with a minimum probability $\alpha$ during the time interval $T$. The objective is to minimize the number of nodes to accomplish a task and two algorithms are proposed, namely inter-location and inter-meeting-time. In the first case, the algorithm selects a minimal group of nodes by considering the reciprocal distance. The second one considers the expected meeting time between the nodes.

Tasks are typically expected to be fulfilled before a deadline. This problem is considered in [202], where the participants perform tasks with a given probability. Cooperation among the participants to accomplish a common task ensures that the completion deadline is respected. The deadline-sensitive user recruitment problem is formalized as a set cover problem with non-linear programming constraints, which is NP-hard. In [200], the authors focus on the allocation of location-dependent tasks, which is a NP-hard and propose a local-ratio-based algorithm (LRBA). The algorithm divides the whole problem into several sub-problems through the modification of the reward function at each iteration. In [205] the authors aim to maximize the spatial distribution and visual correlation while selecting geo-tagged photos with the maximum utility. The KL divergence-based clustering can be employed to distinguish uncertain objects with multiple features [204]. The symmetry KL divergence and Jensen-Shannon KL divergence are seen as two different mutated forms to improve the proposed algorithm.

Task allocation. The problem of task allocation defines the methodologies of task dispatching and assignment to the participants. TaskMe [209] solves two bi-objective optimization problems for multi-task user selection. One considers the case of a few participants with several tasks to accomplish. The second examines the case of several participants with few tasks. iCrowd is a unified task allocation framework for piggyback crowdsensing [208] and defines a novel spatialtemporal coverage metric, i.e., the $k$-depth coverage which considers both the fraction of subareas covered by sensor readings and the number of samples acquired in each covered sub-area. iCrowd can operate to maximize the overall $k$-depth coverage across a sensing campaign with a fixed budget or to minimize the overall incentive payment while ensuring a predefined $k$-depth coverage constraint. Xiao et al. analyze the task assignment problem following mobility models of mobile social networks [210]. The objective is to minimize the average makespan. Users while moving can re-distribute tasks to other users via Bluetooth or WiFi that will deliver the result during the next meeting. Data redundancy can play an important role in task allocation. To maximize the aggregate data utility while performing heterogeneous sensing tasks being budget-constrained, [211] proposes a fairness-aware distributed algorithm that takes data redundancy into account to refine the relative importance of tasks over time. The problem is subdivided it into two subproblems, i.e., recruiting and allocation. For the former, a greedy algorithm is proposed. For the latter, a dual-based decomposition technique is enforced to determine the workload of different tasks for each user. Wang et al. [207] propose a MCS task allocation that aligns task sequences with citizens' mobility. By leveraging the repetition of mobility patterns, the task allocation problem is studied as a pattern matching problem and the optimization aims to pattern matching length and support degree metrics. Fair task allocation and energy efficiency are the main focus of [212], whose objective is to provide min-max aggregate sensing time. The problem is NP-hard and it is solved with a polynomial-time approximation algorithm (for the offline case) and with a polynomial-time greedy algorithm (for the online case). With Crowdlet [213], the demands for sensing data of users, called requesters are satisfied by tasking other users in the neighborhood for a fast response. The model determines expected service gain that a requester can experience when recruiting another user. The problem then turns into how to maximize the expected sum of service quality and Crowdlet proposes an optimal worker recruitment policy through the dynamic programming principle.

Proper workload distribution and balancing among the participants is important. In [206], the authors investigate the trade-off between load balance and data utility maximization by modeling workload allocation as a Nash bargaining game to determine the workload of each smartphone. The heterogeneous Multi-Task Assignment (HMTA) problem is presented and formalized in [214]. The authors aim to maximize the quality of information while minimizing the total incentive budget. They propose a problem-solving approach of two stages by exploiting the spatio-temporal correlations among several heterogeneous 
and concurrent tasks in a shared resource pool. In [215], the authors investigate the problem of location diversity in MCS systems, where tasks arrive stochastically while citizens are moving. In the offline scenario, a combinatorial algorithm efficiently allocates tasks. In online scenarios, the authors investigate the stochastic characteristics and discontinuous coverage to address the challenge of non-linear expectation and provide fairness among users. In [216] the authors review the task allocation problem by focusing on task completion in urban environments and classifying different allocation algorithms according to related problems. Often, organizers want to minimize the total costs of incentives. To this end, a cost-efficient incentive mechanism is presented in [217] by exploring multi-tasking versus single-task assignment scenarios.

User recruitment. Proper user recruitment defines the degree of effectiveness of a MCS system, hence this problem has largely been investigated in analytical research works.

In [218], the authors propose a novel dynamic user recruitment problem with heterogeneous sensing tasks in a largescale MCS system. They aim at minimizing the sensing cost while satisfying a certain level of coverage in a context with dynamic tasks which change in real-time and heterogeneous with different spatial and temporal requirements. To this scope, they propose three greedy algorithms to tackle the dynamic user recruitment problem and conduct simulations exploiting a real mobile dataset. Zhang et al. [219] analyze a real-world SS7 data of 1.18 million mobile users in Xiamen, China and address a mobile user recruitment problem. Given a set of target cells to be sensed and the recruitment cost functions of the mobile users, the MUR problem is to recruit a set of participants to cover all the cells and minimize the total recruitment cost. In [220], the authors analyze how participants may be optimally selected, focusing on the coverage dimension of the sensing campaign design problems and the complexity of opportunistic and delay tolerant networks. Assuming that transferring data as soon as generated from the mobile devices' sensors is not the best way for data delivery, they consider scenarios with deterministic node mobility and propose the choice of participants as a minimum cost set cover problem with the sub-modular objective function. They describe practical data heuristics for the resulting NP-hard problems and in the experimentation with real mobility datasets provide evidence that heuristics perform better than worst case bound suggest. The authors of [221] investigate incentive mechanisms considering only the crucial dimension of location information when tasks are assigned to users. TRAC (Truthful Auction for Location-Aware Collaborative sensing) is a mechanism that is based on a reverse auction framework and consists of a near-optimal approximate algorithm and a critical payment scheme. CrowdRecruiter [222] and CrowdTasker [223] aim to select the minimal set of participants under probabilistic coverage constraints while maximizing the total coverage under a fixed budget. They are based on a prediction algorithm that computes the coverage probability of each participant and then exploits a near optimal function to measure the joint coverage probability of multiple users. In [224], the authors propose incentive mechanisms that can be exploited to motivate participants in sensing campaigns. They consider two different approaches and propose solutions accordingly. In the first case, the responsible for the sensing campaign provides a reward to the participants, which they model as a Stackelberg game in which users are the followers. In the other case, participants directly ask an incentive for their service, for which the authors design an auction mechanism, called IMCU. ActiveCrowd is a user selection framework for multi-task MCS architectures [225]. The authors analyze the problem under two situations: users' selection based on intentional individuals' movement for time-sensitive tasks and unintentional movement for delay-tolerant tasks. In order to accomplish time-sensitive tasks, users are required to move to the position of the task intentionally and the aim is to minimize the total distance. In the case of delay-tolerant tasks, the framework chooses participants that are predicted to pass close to the task and the aim is to minimize the number of required users. The authors propose to solve the two problems of minimization exploiting two Greedy-enhanced genetic algorithms. A method to optimally select users to generate the required space-time paths across the network for collecting data from a set of fixed locations is proposed in [226]. Finally, one among the first works proposing incentives for crowdsourcing considers two incentive methods, a platform-centric model and a user-centric model [227]. In the first one, the platform supplies a money contribution shared by participants by exploiting a Stackelberg game. In the second one, users have more control over the reward they will get through an auction-based incentive mechanism.

Context awareness. MCS systems are challenged by the limited amount of resources in mobile devices in terms of storage, computational, and communication capabilities. Context-awareness allows to react more smartly and proactively to changes in the environment around mobile devices [228] and influences energy and delay trade-offs of MCS systems [229]. In [230], the authors focus on the trade-offs between energy consumption due to continuous sensing and sampling rate to detect contextual events. The proposed Viterbi-based ContextAware Mobile Sensing mechanism optimizes sensing decisions by adaptively deciding when to trigger the sensors. For contextbased mobile sensing in smart building, [231] exploits low power sensors and proposes a model that enhances communication, data processing, and analytics. In [232], the authors propose a multidimensional context-aware social network architecture for MCS applications. The objective is to provide context-aware solutions of environmental, personal, and social information for both customer and contributing users in smart cities applications. In [233], the authors propose a contextaware approximation algorithm to search the optimal solution for a minimum vertex cover NP-complete problem that is tailored for crowdsensing task assignment. Understanding the physical activity of users while performing data collection is as-well-important as context-awareness. To this end, recent works have shown how to efficiently analyze sensor data to recognize physical activities and social interactions using machine learning techniques [234], [235].

Budget-constrained. In MCS systems data collection is performed by non-professional users with low-cost sensors. Consequently, the quality of data cannot be guaranteed. To 
obtain effective results, organizers typically reward users according to the amount and quality of contributed data. Thus, their objective is to minimize the budget expenditure while maximizing the amount and quality of gathered data.

BLISS (Budget LImited robuSt crowdSensing) [112] is an online learning algorithm that minimizes the difference between the achieved total revenue and the optimal one. It is asymptotically as the minimization is performed on average. Prediction-based user recruitment strategy in [237] divides contributing users in two groups, namely, pay as you go (PAYG) and pay monthly (PAYM). Different price policies are proposed in order to minimize the data uploading cost. The authors of [236] consider only users that are located in a space-temporal vicinity of a task to be recruited. The aim is to maximize the number of accomplished tasks under the budget constraint. Two approaches to tackle the problem are presented. In the first case, the budget is given for a fixed and short period, while in the second case it is given for the entire campaign. The authors show that both variants are NP-hard and present different heuristics and a dynamic budget allocation policy in the online scenario. ALSense [238] is a distributed active learning framework that minimizes the prediction errors for classification-based mobile crowdsensing tasks subject to data upload and query cost constraints. ABSee is an auction-based budget feasible mechanism that aims to maximize the quality of sensing of users [239]. Its design is based on a greedy approach and includes winner selection and payment determination rules. Another critical challenge for campaign organizers is to set a proper posted price for a task to be accomplished with small total payment and sufficient quality of data. To this end, [240] proposes a mechanism based on a binary search to model a series of chance constrained posted pricing problems in robust MCS systems.

\section{Platforms, Simulators and Datasets}

In the previous part of this Section, we presented MCS as a layered architecture, illustrated operation of DCFs to acquire data from citizens and analytic works that address issues of MCS systems theoretically. Now, we discuss practical research works. As shown in Table VI, we first present platforms for data collection that provide resources to store, process, and visualize data. Then, we discuss existing simulators that focus on different aspects of MCS systems. Finally, we analyze some existing and publicly available collected datasets.

While the main objective of platforms is to enable researchers to experiment applications in real-world, simulators are better suited to test the technical performance of specific aspect of a MCS system, for example, the techniques for data reporting. Simulators have the advantage of operating at large scale at the expense of non-realistic settings, while platforms are typically limited in the number of participants. Datasets are collected through real-world experiments that involve participants to sense and contribute data typically through a custom developed mobile application. The importance of datasets lies in the fact that enable researchers to perform studies on the data or to employ as inputs for simulators.
Platforms. Participact is a large-scale crowdsensing platform that allows the development and deployment of experiments, considering both mobile device and server side [242]. It considers not only technical issues but also human resources and their involvement. APISENSE is a popular cloud-based platform that enables researchers to deploy crowdsensing applications by providing resources to store and process data acquired from a crowd [243]. It presents a modular serviceoriented architecture on the server-side infrastructure that allows researchers to customize and describe requirements of experiments through a scripting language. Also, it makes available to the users other services (e.g., data visualization) and a mobile application, allowing them to download the tasks, to execute them in a dedicated sandbox and to upload data on the server automatically. SenseMyCity is a MCS platform that consists of an infrastructure to acquire geo-indexed data through mobile devices' sensors, exploiting a multitude of voluntary users willing to participate in the sensing campaign and the logistic support for large-scale experiments in urban environments. It is capable of handling data collected from different sources, such as GPS, accelerometer, gyroscope, and environmental sensors, either embedded or external [244]. CRATER is a crowdsensing platform to estimate road conditions [245]. It provides APIs to access data and visualize maps in the related application. Medusa is a framework that provides high-level abstractions for analyzing the steps to accomplish a task by users [246]. It exploits a distributed runtime system that coordinates the execution and the flow control of these tasks between users and a cluster on the cloud. Prism is a Platform for Remote Sensing using Smartphones that balances generality, security, and scalability [247]. MOSDEN is a Mobile Sensor Data EngiNe used to capture and share sensed data among distributed applications and several users [63]. It is scalable, exploits cooperative opportunistic sensing and separates the application processing from sensing, storing, and sharing. Matador is a platform that focuses on energy-efficient task allocation and execution based on users context awareness [248]. To this end, it aims to assign tasks to users according to their surrounding preserving the normal smartphone usage. It consists of a design and prototype implementation that includes a context-aware energy-efficient sampling algorithm aiming to deliver MCS tasks by minimizing energy consumption.

Simulators. The success of a MCS campaign relies on a large participation of citizens [255]. Hence, often it is not feasible to deploy large-scale testbeds and it is preferable to run simulations with a precise set-up in realistic urban environments. Currently, the existing tools aim either to well characterize and model communication aspects or define the usage of spatial environment [256]. CrowdSenSim is a new tool to assess the performance of MCS systems and smart cities services in realistic urban environments [68]. For example, it has been employed for analysis of city-wide smart lighting solutions [257]. It is specifically designed to perform analysis in large scale environments and supports both participatory and opportunistic sensing paradigms. This custom simulator implements independent modules to characterize the urban environment, the user mobility, the communication and 
TABLE VI

PLATFORMS, SIMULATORS AND DATASETS

\begin{tabular}{|c|c|c|c|}
\hline & WORKS & DESCRIPTION & REFERENCE \\
\hline \multirow[t]{8}{*}{ PLATFORMS } & ParticipAct Living Lab & $\begin{array}{l}\text { It is a large-scale crowdsensing platform that allows the development and } \\
\text { deployment of experiments, considering both mobile device and server side }\end{array}$ & [242] \\
\hline & APISENSE & $\begin{array}{l}\text { It enables researchers to deploy crowdsensing applications by providing resources } \\
\text { to store and process data acquired from a crowd }\end{array}$ & [243] \\
\hline & SenseMyCity & $\begin{array}{l}\text { It acquires geo-tagged data acquired from different mobile devices' sensors of } \\
\text { users willing to participate in experiments }\end{array}$ & [244] \\
\hline & CRATER & $\begin{array}{l}\text { It provides APIs to access data and visualize maps in the related application to } \\
\text { estimate road conditions }\end{array}$ & [245] \\
\hline & Medusa & $\begin{array}{l}\text { It provides high level abstractions for analyzing the required steps to accomplish } \\
\text { a task by users }\end{array}$ & [246] \\
\hline & PRISM & $\begin{array}{l}\text { Platform for Remote Sensing using Smartphones that balances generality, security } \\
\text { and scalability }\end{array}$ & [247] \\
\hline & MOSDEN & $\begin{array}{l}\text { It is used to capture and share sensed data among distributed applications and } \\
\text { several users }\end{array}$ & [63] \\
\hline & MATADOR & $\begin{array}{l}\text { It aims to efficiently deliver tasks to users according to a context-aware sampling } \\
\text { algorithm that minimizes energy consumption of mobile devices }\end{array}$ & [248] \\
\hline \multirow[t]{4}{*}{ SimulatoRS } & CrowdSenSim & $\begin{array}{l}\text { It simulates MCS activities in large-scale urban environments, implementing } \\
\text { DCFs and realistic user mobility }\end{array}$ & [68] \\
\hline & NS-3 & $\begin{array}{l}\text { Used in a MCS environment considering mobility properties of the nodes and } \\
\text { the wireless interface in ad-hoc network mode }\end{array}$ & [66] \\
\hline & CupCarbon & $\begin{array}{l}\text { Discrete-event WSN simulator for IoT and smart cities, which can be used for } \\
\text { MCS purposes taking into account users as mobile nodes and base stations }\end{array}$ & [249] \\
\hline & Urban parking & $\begin{array}{l}\text { It presents a simulation environment to investigate performance of MCS } \\
\text { applications in an urban parking scenario }\end{array}$ & [250] \\
\hline \multirow[t]{5}{*}{ DATASETS } & ParticipAct & $\begin{array}{l}\text { It involves in MCS campaigns } 173 \text { students in the Emilia Romagna region } \\
\text { (Italy) on a period of } 15 \text { months using Android smartphones }\end{array}$ & [64] \\
\hline & Cambridge & $\begin{array}{l}\text { It presents the mobility of } 36 \text { students in the Cambridge University Campus for } \\
12 \text { days }\end{array}$ & [251] \\
\hline & MIT & $\begin{array}{l}\text { It provides the mobility of } 94 \text { students in the MIT Campus (Boston, MA) for } \\
246 \text { days }\end{array}$ & [252] \\
\hline & MDC Nokia & $\begin{array}{l}\text { It includes data collected from } 185 \text { citizens using a N95 Nokia mobile phone } \\
\text { in the Lake Geneva region in Switzerland }\end{array}$ & [253] \\
\hline & CARMA & $\begin{array}{l}\text { It consists of } 38 \text { mobile users in a university campus over several weeks using } \\
\text { a customized crowdsourcing Android mobile application }\end{array}$ & [254] \\
\hline
\end{tabular}

the crowdsensing inputs, which depend on the application and specific sensing paradigm utilized. CrowdSenSim allows scientists and engineers to investigate the performance of the MCS systems, with a focus on data generation and participant recruitment. The simulation platform can visualize the obtained results with unprecedented precision, overlaying them on city maps. In addition to data collection performance, the information about energy spent by participants for both sensing and reporting helps to perform fine-grained system optimization. Network Simulator 3 (NS-3) has been used for crowdsensing simulations to assess the performance of a crowdsensing network taking into account the mobility properties of the nodes together with the wireless interface in ad-hoc network mode [66]. Furthermore, the authors present a case study about how participants could report incidents in public rail transport. NS-3 provides highly accurate estimations of network properties. However, having detailed information on communication properties comes with the cost of losing scalability. First, it is not possible to simulate tens of thousands of users contributing data. Second, the granularity of the duration of NS-3 simulations is typically in the order of minutes. Indeed, the objective is to capture specific behaviors such as the changes in the TCP congestion window. However, the duration of real sensing campaigns is typically in the order of hours or days. CupCarbon is a discrete-event wireless sensor network (WSN) simulator for IoT and smart cities [249]. One of the major strengths is the possibility to model and simulate WSN on realistic urban environments through OpenStreetMap. To set up the simulations, users must deploy on the map the various sensors and the nodes, such as mobile devices and base stations. The approach is not intended for crowdsensing scenarios with thousands of users. In [250], the authors present a simulation environment developed to investigate the performance of crowdsensing applications in an urban parking scenario. Although the application domain is only parkingbased, the authors claim that the proposed solution can be applied to other crowdsensing scenarios. However, the scenario considers only drivers as a type of users and movements from one parking spot to another one. The authors consider humans as sensors that trigger parking events. However, to be widely applicable, a crowdsensing simulator must consider data generated from mobile and IoT devices' sensors carried by human individuals.

Datasets. In literature, it is possible to find different valuable datasets produced by research projects which exploit MCS 
platforms. In these projects, researchers have collected data in various geographical areas through different sets of sensors and with different objectives. These datasets are fundamental for giving the possibility to all the research community providing a way to test, assess and compare several solutions for a wide range of application scenarios and domains. The ParticipAct Living Lab is a large-scale experiment of the University of Bologna involving in crowdsensing campaigns 173 students in the Emilia Romagna region (Italy) on a period of 15 months using Android smartphones [64], [258]. In this work, the authors present a comparative analysis of existing datasets and propose ParticipAct dataset to assess the performance of a crowdsensing platform in user assignment policies, task acceptance, and task completion rate. The MDC Nokia dataset includes data collected from 185 citizens using a N95 Nokia mobile phone in the Lake Geneva region in Switzerland [253]. An application running on the background collects data from different embedded sensors with a sampling period of $600 \mathrm{~s}$. The Cambridge dataset presents the mobility of 36 students in the Cambridge University Campus for 12 days [251]. The only information provided concerns traces of-location among participants, which are taken through a Bluetooth transceiver in an Intel iMote. The MIT dataset provides the mobility of 94 students in the MIT Campus for 246 days [252]. The users exploited an application that took into account co-location with other participants through Bluetooth and other data, but no sensor data. CARMA is a crowdsourcing Android application used to perform an experimental study and collect a dataset used to derive empirical models for user mobility and contact parameters, such as frequency and duration of user contacts [254]. It has been collected through two stages, the first with 38 mobile users contributing for 11 weeks and the second with 13 students gathering data over 4 weeks. In addition, participants filled a survey to investigate the correlation of mobility and connectivity patterns with social network relations

\section{E. MCS as a Business Paradigm}

Data trading has recently attracted a remarkable and increasing attention. The analysis of information acquisition from the point of view of data markets is still in its infancy for both industry and research. In this context, MCS acts as a business paradigm where citizens are at the same time data contributors, customers, and service consumers. This eco-system requires new design strategies to enforce efficiency of MCS systems.

VENUS [259] is a profit-driVEN data acqUiSition framework for crowd-sensed data markets that aims to provide crowdsensed data trading format determination, profit maximization with polynomial computational complexity, and payment minimization in strategic environments. How to regulate the transactions between users and the MCS platform requires further investigation. To give some representative examples, [260] proposes a trusted contract theory-based mechanism to provide sensing services with incentive schemes. The objective is, on the one hand, to guarantee the quality of sensing while maximizing the utility of the platform, and on the other hand to satisfy users with rewards. A collaboration mechanism based on rewarding is also proposed in [261], where the organizer announces a total reward to be shared between contributors. A design of data sharing market and a generic pricing scheme are presented in [262], where contributors can sell their data to customers that are not willing to collect information on their own. In this work, the authors prove the existence and uniqueness of the market equilibrium and propose a quality-aware P2P-based MCS architecture for such a data market. In addition, they present iterative algorithms to reach the equilibrium and discuss how P2P data sharing can enhance social welfare by designing a model with low trading prices and high transmission costs. Data market in P2P-based MCS systems is also studied in [263] that propose a hybrid pricing mechanism to reward contributors.

When the aggregated information is made available as a public good, a system can also benefit from merging data collection with routing selection algorithms through incentive mechanisms. As the utility of collected information increases with the diversity of users' paths, it is crucial to reward participants accordingly. For example, users that move apart from the target path contributing data should receive a higher reward than users who do not. To this end, in [264] proposes two different rewarding mechanisms to tradeoff path diversity and user participation, motivating a hybrid mechanism to increase social welfare. In [265], the authors investigate the economics of delay-sensitive MCS campaigns by presenting an Optimal Participation and Reporting Decisions (OPRD) algorithm that aims to maximize the service provider's profit. In [266], the authors study market behaviors and incentive mechanisms by proposing a non-cooperative game under a pricing scheme for a theoretical analysis of the social welfare equilibrium.

The cost of data transmission is one of the most influencing factors that lower user participation. To this end, a contractbased incentive framework for WiFi community networks is presented in [267], where the authors study the optimal contract and the profit gain of the operator. The operators augment their profit by increasing the average WiFi access quality. Although counter-intuitive, this process lowers prices and subscription fees for end-users. In [268] the authors discuss MCS for industrial applications by highlighting benefits and shortcomings. In this area, MCS can provide an efficient, costeffective and scalable data collection paradigm for industrial sensing to boost performance in connecting the components of the entire industrial value chain.

\section{F. Final Remarks}

We proposed to classify MCS literature works in a fourlayered architecture. The architecture enables detailed categorization of all areas of MCS, from sensing to the application layer, including communication and data processing. In addition, it allows to differentiate between domain-specific and general-purpose frameworks for data collection. We have discussed both theoretical works, including those pertaining to the areas of operational research and optimization (e.g., how to maximize accomplished tasks under budget constraints) and practical ones, such as platforms, simulators, and existing datasets collected by research projects. In addition, we have discussed MCS as a business paradigm where data is traded as a good. 


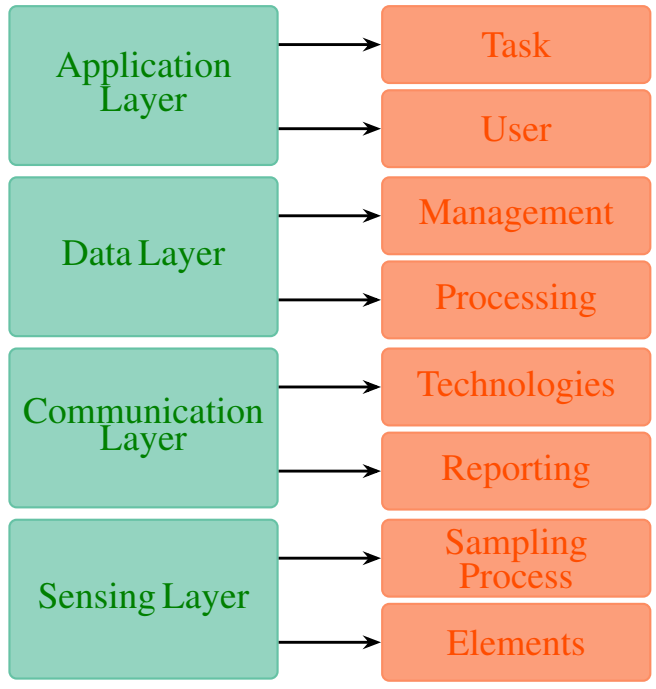

Fig. 9. Taxonomies on MCS four-layered architecture. It includes sensing, communication, data, and application layers. Sensing layer is divided between sampling and elements, which will be described in Sec. VII. Communication layer is divided between technologies and reporting, which will be discussed in Sec. VI. Data layer is divided between management and processing, and will be presented in Sec. V. Application layer is divided between task and user, which will be discussed in Sec. IV.

In the next part of our manuscript, we take the organization of the vast literature on MCS one step further by proposing novel taxonomies that build on and expand the discussion on the previously introduced layered architecture. In a nutshell, we i) propose new taxonomies by subdividing each layer into two parts, as shown in Fig. 9, ii) classify the existing works according to the taxonomies and, iii) exemplify the proposed taxonomies and classifications with relevant cases. The ultimate objective is to classify the vast amount of still uncategorized research works and establish consensus on several aspects or terms currently employed with different meanings. For the classification, we resort to classifying a set of relevant research works that we use to exemplify the taxonomies. Note that, for space reasons, we will not introduce beforehand these works with a detailed description.

The outline of the following Sections is as follows. The application layer, composed of task and user taxonomies, is presented in Sec. IV. The data layer, composed of management and processing taxonomies, is presented in Sec. V. The communication layer, composed of technologies and reporting taxonomies, is presented in Sec. VI. Finally, the sensing layer, composed of sampling process and elements taxonomies, will be discussed in Sec. VII.

\section{TAXONOMIES AND ClASSIFICATION ON APPLICATION LAYER}

This Section analyzes the taxonomies of the application layer This layer is mainly composed by task and user categories, which are discussed with two corresponding taxonomies (Subsection IV-A). Then, we classify papers accordingly (Subsection IV-B). Fig. 10 shows the taxonomies on the application layer.

\section{A. Taxonomies}

1) Task: The upper part of Fig. 10 shows the classification of task-related taxonomies, which comprise pro-active and reactive scheduling, methodologies for task assignment and execution.

Scheduling. Task scheduling describes the process of allocating the tasks to the users. We identify two strategies for the assignment, on the basis of the behavior of a user. With proactive scheduling, users that actively sense data without a pre-assigned task, e.g., to capture a picture. Reactive scheduling indicates that users receive tasks and contribute data to accomplish the received jobs.

Proactive. Under pro-active task scheduling, users can freely decide when, where, and when to contribute. This approach is helpful for example in the public safety context to receive information from users that have assisted to a crash accident. Several social networks-based applications assign tasks only after users have already performed sensing [59], [149].

Reactive. Under reactive task scheduling, a user receives a request and, upon acceptance, accomplishes a task. Tasks should be assigned in a reactive way when the objective to be achieved is already clear before starting the sensing campaign. To illustrate with an example, monitoring a phenomenon like air pollution [56] falls in this category.

Assignment. It indicates how tasks are assigned to users. According to the entity that dispatches tasks, we classify assignment processes into centralized where there is a central authority and decentralized where users themselves dispatch tasks [15].

Central authority. In this approach, a central authority distributes tasks among users. Typical centralized task distributions involve environmental monitoring applications, such as detection of ionosphere pollution [128] or nuclear radiation [120].

Decentralized. When the task distribution is decentralized, each participant becomes an authority and can either perform the task or to forward it to other users. This approach is very useful when users are very interested in specific events or activities. A typical example is Mobile Social Network or Intelligent Transportation Systems, to share news on public transport delays [142] or comparing prices of goods in real-time [133].

Execution. This category defines the methodologies for task execution.

Single task. Under this category fall those campaigns where only one type of task is assigned to the users, for instance taking a picture after a car accident or measuring the level of decibel for noise monitoring [123].

Multi-tasking. On the contrary, a multi-tasking campaign execution foresees that the central authority assigns multiple types of tasks to users. To exemplify, simultaneous monitoring of noise and air quality.

2) User: The lower part of Fig. 10 shows the user-related taxonomies that focus on recruitment strategy, selection criteria, and type of users. 


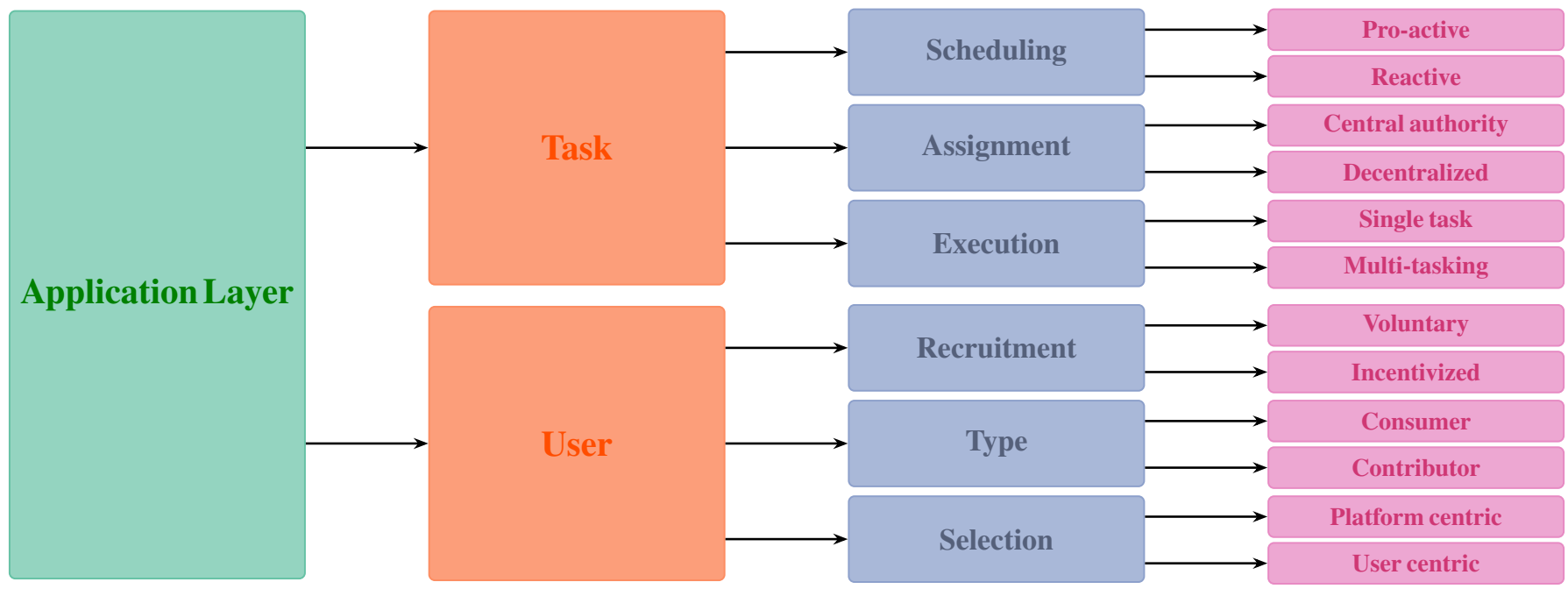

Fig. 10. Taxonomies on application layer, which is composed of task and user categories. The task-related taxonomies are composed of scheduling, assignment and execution categories, while user-related taxonomies are divided into recruitment, type and selection categories.

Recruitment. Citizen recruitment and data contribution incentives are fundamental to the success of MCS campaigns. In literature, the term user recruitment is used with two different meanings. The first, and the most popular is related to citizens who join a sensing campaign and become potential contributors. The second meaning is less common but is employed in more recent works and indicates the process of selecting users to undertake a given task from the pool of all contributors. To capture such a difference, we introduce and divide the terms user recruitment and user selection, as illustrated in Fig.11. In our taxonomy, user recruitment constitutes only the process of recruiting participants, who can join on a voluntary basis or through incentives. Then, sensing tasks are allocated to the recruited users according to policies specifically designed by the sensing campaign organizer and contributing users are selected between them. We will discuss user selection in the following paragraph.

The strategies to obtain large user participation are strictly related to the type of application. For example, for a healthcare application that collects information on food allergies (or gluten-free [116]), people affected by the problem usually volunteer to participate. However, if the application target is more generic and does not match well with users' interests (e.g., noise monitoring [123]), the best solution is to encourage user participation through incentive mechanisms. It should be noted that these strategies are not mutually exclusive and users can first volunteer and then be rewarded for quality execution of sensing tasks. Such a solution is well suited for cases when users should perform additional tasks (e.g., sending more accurate data) or in particular situations (e.g., few users contribute from a remote area).

Voluntary participation. Citizens can volunteer to join a sensing campaign for personal interests (e.g., when mapping restaurants and voting food for celiacs or in the healthcare) or willingness to help the community (e.g., air quality and pollution [56]). In volunteer-built MCS systems users are willing to participate and contribute spontaneously without expecting to receive

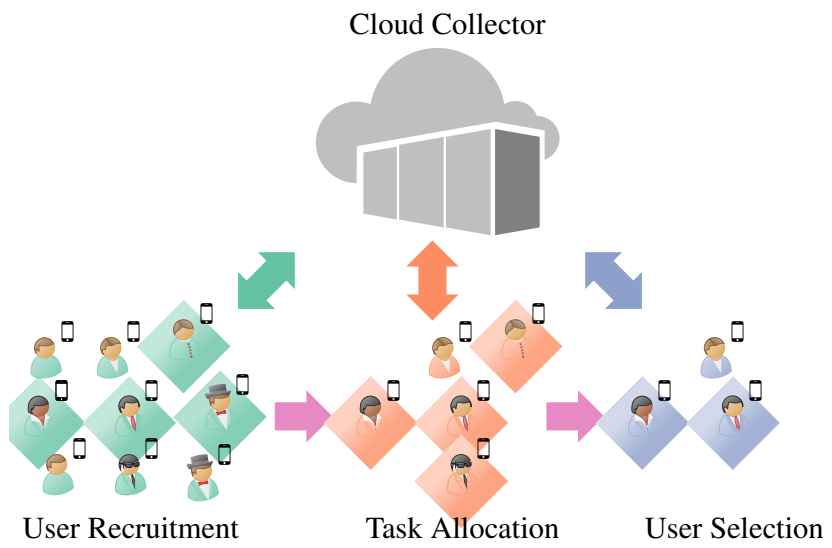

Fig. 11. User recruitment and user selection process. The figure shows that users are recruited between citizens, then tasks are tentatively allocated to users, which can accept or not. Upon acceptance, users are selected to contribute data to the central collector.

monetary compensation. Apisense is an example of a platform helping organizers of crowdsensing systems to collect data from volunteers [269].

Recruitment through incentives. To promote participation and control the rate of recruitment, a set of user incentives can be introduced in MCS [227], [270]-[272]. Many strategies have been proposed to stimulate participation [11], [15], [273]. It is possible to classify the existing works on incentives into three categories [12]: entertainment, service, and money. The entertainment category stimulates people by turning some sensing tasks into games. The service category consists in rewarding personal contribution with services offered by the system. Finally, monetary incentives methods provide money as a reward for users' contribution. In general, incentives should also depend on the quality of contributed data and decided on the relationship between demand and supply, e.g., applying microeconomics concepts [274]. MCS systems may consider distributing incentives in an online or offline scenario [275].

Selection. User selection consists in choosing contributors to 
a sensing campaign that better match its requirements. Such a set of users is a subset of the recruited users. Several factors can be considered to select users, such as temporal or spatial coverage, the density of users in certain regions of interest or variable user availability. We mainly classify the process of user selection through the classes user centric and platform centric.

User centric. When the selection is user centric, contributions depend only on participants willingness to sense and report data to the central collector, which is not responsible for choosing them.

Platform centric. User selection is platform centric when the central authority directly decides data contributors. The decision is usually based on parameters decided by organizers, such as temporal and spatial coverage, the total amount of collected data, mobility of users, or density of users and data in a particular region of interests. In other words, platform centric decisions are taken according to the utility of data to accomplish the targets of the campaign.

Type. This category classifies users into contributors and consumers. Contributors are individuals that sense and report data to a collector, while the consumers utilize sensed data without having contributed. A user can also join a campaign assuming both roles. For instance, users can send pictures with the price of fuel when they pass by a gas station (contributors), while at the same time the service shows fuel prices at the nearby gas stations (consumer) [131].

Contributor. A contributor reports data to the MCS platform with no interest in the results of the sensing campaign. Contributors are typically driven by incentives or by the desire to help the scientific or civil communities (e.g., a person can collect data from the microphone of a mobile device to map noise pollution in a city [115] with no interests of knowing results).

Consumer. Citizens who join a service to obtain information about certain application scenario and have a direct interest in the results of the sensing campaign are consumers. Celiac people, for instance, are interested in knowing which restaurants to visit [116].

\section{B. Classification}

This part classifies and surveys literature works according to the task- and user-related taxonomies previously proposed in this Section.

Task. The following paragraphs survey and classify research works according the task taxonomies presented in IV-A1. Table VII illustrates the classification per paper.

Scheduling. A user contributes data in a pro-active way according to his/her willingness to sense specific events (e.g., taking a picture of a car accident). Typical pro-active applications are in health care [17], [19], [136], and mobile social networks [59], [60], [151], [152], [155] domains. Other examples are sharing prices of goods [132], [133], and recommending information to others, such as travelling advice [169]. Reactive scheduling consists in sending data according to a pre-assigned task, such as traffic [20] conditions detection, air quality [21], and noise monitoring [115], [121]-[123]. Other examples consist in conferences [167], emotional situations [149], detecting sounds [166], characterizing WiFi coverage [164], space weather [129]. While in MSNs applications are typically a reactive scheduling, Whozthat [153] and MobiClique [150] represent two excpetions.

Assignment. Task assignment can be centralized or decentralized according to the entity that performs the dispatch. Tasks are typically assigned by a central authority when data aggregation is needed to infer information, such as for monitoring traffic [20], [145] conditions, air pollution [21], noise [115], [122], [123], [166], and weather [129] Tasks assignment is decentralized when users are free to distribute tasks among themselves in a peer-to-peer fashion, for instance sending pictures of a car accident as alert messages [143]. Typical decentralized applications are mobile social networks [59], [60], [60], [150]-[153] or sharing info about services [132], [133], [169],

Execution. Users can execute one or multiple tasks simultaneously in a campaign. Single task execution includes all MCS campaigns that require users to accomplish only one task, such as mapping air quality [21] or noise [115], [122], [123], and detecting prices [132], [133]. The category multi-tasking specifies works in which users can contribute multiple tasks at the same time or in different situations. For instance, mobile social networks imply that users collect videos, images, audios related to different space and time situations [59], [60], [60], [150], [155], Healthcare applications usually also require a multitasking execution, including different sensors to acquire different information [17], [19], [20], such as images, videos, or physical activities recognized through activity pattern during a diet [136].

User. The following paragraphs survey and classify research works according to the user-related taxonomies on recruitment and selection strategies, and type of users presented in IV-A2. Table VIII shows the classification per paper.

Recruitment. It classifies as voluntary when users do not receive any compensation for participating in sensing and when they are granted with compensation through incentive mechanisms, which can include monetary rewarding, services or other benefits. Typical voluntary contributions are in health care applications [17], [19], [136], such as celiacs who are interested in checking and sharing gluten-free food and restaurants [116]. Users may contribute voluntary also when monitoring phenomena in which they are not only contributors but also interested consumers, such as checking traffic and road conditions [20], [142], [143], [145], environment [122], [123] or comparing prices of goods [131], [132]. Mobile social networks are also based on voluntary interactions between users [59], [60], [121], [150], [151], [153], [155] Grant users through incentives is the simplest way to have a large amount of contributed data. Most common incentive is monetary rewarding, which is needed when users are not directly interested in contributing, for instance monitoring noise [115], air pollution [21], and space weather [129]. 
TABLE VII

CLASSIFICATION BASED ON TASK TAXONOMIES OF APPLICATION LAYER

\begin{tabular}{|c|c|c|c|c|c|c|c|}
\hline \multirow[b]{2}{*}{ PROJECT } & \multirow[b]{2}{*}{ REFERENCE } & \multicolumn{2}{|c|}{ SCHEDULING } & \multicolumn{2}{|c|}{ ASSIGNMENT } & \multicolumn{2}{|c|}{ EXECUTION } \\
\hline & & Pro-active & Reactive & Central Aut. & Decentralized & Single task & Multi-tasking \\
\hline HealthAware & [17] & $\mathrm{x}$ & & $\mathrm{x}$ & & & $\mathrm{x}$ \\
\hline DietSense & [19] & $\mathrm{x}$ & & $\mathrm{x}$ & & & $\mathrm{x}$ \\
\hline Nericell & [20] & & $\mathrm{x}$ & $\mathrm{x}$ & & & $\mathrm{x}$ \\
\hline NoiseMap & [115] & & $\mathrm{x}$ & $\mathrm{x}$ & & $\mathrm{x}$ & \\
\hline GasMobile & [21] & & $\mathrm{x}$ & $\mathrm{x}$ & & $\mathrm{x}$ & \\
\hline NoiseTube & [123] & & $\mathrm{x}$ & $\mathrm{x}$ & & $\mathrm{x}$ & \\
\hline CenceMe & [59] & $\mathrm{x}$ & & & $\mathrm{x}$ & & $\mathrm{x}$ \\
\hline MicroBlog & {$[60]$} & $\mathrm{x}$ & & & $\mathrm{x}$ & & $\mathrm{x}$ \\
\hline PEIR & [121] & & $\mathrm{x}$ & $\mathrm{x}$ & & & $\mathrm{x}$ \\
\hline How long to wait? & [142] & $\mathrm{x}$ & & & $\mathrm{x}$ & & $\mathrm{x}$ \\
\hline PetrolWatch & [131] & & $\mathrm{x}$ & & $\mathrm{x}$ & $\mathrm{x}$ & \\
\hline AndWellness & [136] & $\mathrm{x}$ & & & $\mathrm{x}$ & & $\mathrm{x}$ \\
\hline Darwin & [155] & $\mathrm{x}$ & & & $\mathrm{x}$ & & $\mathrm{x}$ \\
\hline CrowdSense@Place & [147] & & $\mathrm{x}$ & $\mathrm{x}$ & & & $\mathrm{x}$ \\
\hline ILR & [168] & $\mathrm{x}$ & & & $\mathrm{x}$ & & $\mathrm{x}$ \\
\hline SoundSense & [166] & & $\mathrm{x}$ & $\mathrm{x}$ & & $\mathrm{x}$ & \\
\hline Urban WiFi & [164] & & $\mathrm{x}$ & $\mathrm{x}$ & & $\mathrm{x}$ & \\
\hline LiveCompare & [133] & $\mathrm{x}$ & & & $\mathrm{x}$ & $\mathrm{x}$ & \\
\hline MobiClique & [150] & & $\mathrm{x}$ & & $\mathrm{x}$ & & $\mathrm{x}$ \\
\hline MobiShop & [132] & $\mathrm{x}$ & & & $\mathrm{x}$ & $\mathrm{x}$ & \\
\hline SPA & [134] & $\mathrm{x}$ & & & $\mathrm{x}$ & & $\mathrm{x}$ \\
\hline EmotionSense & [149] & & $\mathrm{x}$ & $\mathrm{x}$ & & & $\mathrm{x}$ \\
\hline ConferenceSense & [167] & & $\mathrm{x}$ & $\mathrm{x}$ & & & $\mathrm{x}$ \\
\hline Travel Packages & [169] & $\mathrm{x}$ & & & $\mathrm{x}$ & & $\mathrm{x}$ \\
\hline Mahali & [129] & & $\mathrm{x}$ & $\mathrm{x}$ & & $\mathrm{x}$ & \\
\hline Ear-Phone & [122] & & $\mathrm{x}$ & $\mathrm{x}$ & & $\mathrm{x}$ & \\
\hline WreckWatch & [143] & $\mathrm{x}$ & & & $\mathrm{x}$ & & $\mathrm{x}$ \\
\hline VTrack & [145] & & $\mathrm{x}$ & $\mathrm{x}$ & & & $\mathrm{x}$ \\
\hline Social Serendipity & [151] & $\mathrm{x}$ & & & $\mathrm{x}$ & & $\mathrm{x}$ \\
\hline SociableSense & [152] & $\mathrm{x}$ & & & $\mathrm{x}$ & & $\mathrm{x}$ \\
\hline WhozThat & [153] & & $\mathrm{x}$ & & $\mathrm{x}$ & $\mathrm{x}$ & \\
\hline MoVi & [154] & & $\mathrm{x}$ & $\mathrm{x}$ & & $\mathrm{x}$ & \\
\hline
\end{tabular}

Selection. It overviews works between user or platform centric selection. User selection is based on contributors willingness to sense and report data. Voluntary-based applications with interested contributors typically employ this approach, such as mobile social networks [59], [60], [155], comparing live pricing [131]-[133], wellbeing [17], [136], and monitoring traffic [20], [142]. Platform centric selection is used when the central authority requires a specific sensing coverage or type of users. To illustrate with few examples, in [276] the organizers can choose well-suited participants according to specific features such as their habits, and data collection is based on their geographical and temporal availability. In [61] the authors propose a geo-social model that selects the contributors according to a matching algorithm. Other frameworks extend the set of criteria for recruitment [277], including parameters such as the distance between a sensing task and users, their willingness to perform the task and remaining battery life. Nericell [20] collects data of road and traffic condition from specific road segments in Bangalore, while Gas Mobile [21] took measurements of air quality from a set of bicycles moving around several bicycle rides all around the considered city.
Type. It classifies users between consumers, who use a service provided by MCS organizer (e.g., sharing information about food [116]) and contributors, who sense and report data without using the service. In all the works we considered users behave mainly as contributors because they collect data and deliver it to the central authority. In some of the works, users may also act as customers. In health care applications users are typically contributors and consumers, because they not only share personal data but also receive a response or information from other users [17], [19], [136]. Mobile social networks also exploit the fact that users receive collected data from other participants [19], [60], [121], [151], [153], [155] E-commerce is another application that typically leverages on users that are both contributors and consumers [131]-[133]

\section{TAXONOMIES AND ClaSSIFICATION ON DATA LAYER}

This section analyzes the taxonomies on the data layer and surveys research works accordingly. Data layer comprises management and processing. Fig. 12 shows the data-related taxonomies. 
TABLE VIII

CLASSIFICATION BASED ON USER TAXONOMIES OF APPLICATION LAYER

\begin{tabular}{|c|c|c|c|c|c|c|c|}
\hline \multirow[b]{2}{*}{ PROJECT } & \multirow[b]{2}{*}{ REFERENCE } & \multicolumn{2}{|c|}{ RECRUITMENT } & \multicolumn{2}{|c|}{ SELECTION } & \multicolumn{2}{|c|}{ TYPE } \\
\hline & & Voluntary & Incentives & Platform centric & User centric & Consumer & Contributor \\
\hline HealthAware & [17] & $\mathrm{x}$ & & & $\mathrm{x}$ & $\mathrm{x}$ & $\mathrm{x}$ \\
\hline DietSense & [19] & $\mathrm{x}$ & & & $\mathrm{x}$ & $\mathrm{x}$ & $\mathrm{x}$ \\
\hline Nericell & [20] & $\mathrm{x}$ & & $\mathrm{x}$ & & & $\mathrm{x}$ \\
\hline NoiseMap & [115] & & $\mathrm{x}$ & & $\mathrm{x}$ & & $\mathrm{x}$ \\
\hline GasMobile & {$[21]$} & & $\mathrm{x}$ & $\mathrm{x}$ & & & $\mathrm{x}$ \\
\hline NoiseTube & [123] & $\mathrm{x}$ & & $\mathrm{x}$ & & & $\mathrm{x}$ \\
\hline CenceMe & [59] & $\mathrm{x}$ & & & $\mathrm{x}$ & $\mathrm{x}$ & $\mathrm{x}$ \\
\hline MicroBlog & [60] & $\mathrm{x}$ & & & $\mathrm{x}$ & $\mathrm{x}$ & $\mathrm{x}$ \\
\hline PEIR & [121] & $\mathrm{x}$ & & $\mathrm{x}$ & & $\mathrm{x}$ & $\mathrm{x}$ \\
\hline How long to wait? & [142] & $\mathrm{x}$ & & & $\mathrm{x}$ & $\mathrm{x}$ & $\mathrm{x}$ \\
\hline PetrolWatch & [131] & $\mathrm{x}$ & & & $\mathrm{x}$ & $\mathrm{x}$ & $\mathrm{x}$ \\
\hline AndWellness & [136] & $\mathrm{x}$ & & & $\mathrm{x}$ & $\mathrm{x}$ & $\mathrm{x}$ \\
\hline Darwin & [155] & $\mathrm{x}$ & & & $\mathrm{x}$ & $\mathrm{x}$ & $\mathrm{x}$ \\
\hline CrowdSense@Place & [147] & & $\mathrm{x}$ & $\mathrm{x}$ & & & $\mathrm{x}$ \\
\hline ILR & [168] & & $\mathrm{x}$ & & $\mathrm{x}$ & & $\mathrm{x}$ \\
\hline SoundSense & [166] & $\mathrm{x}$ & & & $\mathrm{x}$ & & $\mathrm{x}$ \\
\hline Urban WiFi & [164] & $\mathrm{x}$ & & $\mathrm{x}$ & & & $\mathrm{x}$ \\
\hline LiveCompare & [133] & & $\mathrm{x}$ & $\mathrm{x}$ & & $\mathrm{x}$ & $\mathrm{x}$ \\
\hline MobiClique & [150] & $\mathrm{x}$ & & & $\mathrm{x}$ & $\mathrm{x}$ & $\mathrm{x}$ \\
\hline MobiShop & [132] & $\mathrm{x}$ & & $\mathrm{x}$ & & $\mathrm{x}$ & $\mathrm{x}$ \\
\hline SPA & [134] & $\mathrm{x}$ & & & $\mathrm{x}$ & $\mathrm{x}$ & $\mathrm{x}$ \\
\hline EmotionSense & [149] & $\mathrm{x}$ & & & $\mathrm{x}$ & & $\mathrm{x}$ \\
\hline ConferenceSense & [167] & $\mathrm{x}$ & & & $\mathrm{x}$ & & $\mathrm{x}$ \\
\hline Travel Packages & [169] & & $\mathrm{x}$ & & $\mathrm{x}$ & $\mathrm{x}$ & $\mathrm{x}$ \\
\hline Mahali & [129] & & $\mathrm{x}$ & $\mathrm{x}$ & & & $\mathrm{x}$ \\
\hline Ear-Phone & [122] & $\mathrm{x}$ & & $\mathrm{x}$ & & & $\mathrm{x}$ \\
\hline WreckWatch & [143] & $\mathrm{x}$ & & & $\mathrm{x}$ & & $\mathrm{x}$ \\
\hline VTrack & {$[145]$} & $\mathrm{x}$ & & & $\mathrm{x}$ & $\mathrm{x}$ & $\mathrm{x}$ \\
\hline Social Serendipity & [151] & $\mathrm{x}$ & & & $\mathrm{x}$ & $\mathrm{x}$ & $\mathrm{x}$ \\
\hline SociableSense & {$[152]$} & & $\mathrm{x}$ & & $\mathrm{x}$ & $\mathrm{x}$ & $\mathrm{x}$ \\
\hline WhozThat & [153] & $\mathrm{x}$ & & & $\mathrm{x}$ & $\mathrm{x}$ & $\mathrm{x}$ \\
\hline MoVi & [154] & & $\mathrm{x}$ & $\mathrm{x}$ & & $\mathrm{x}$ & $\mathrm{x}$ \\
\hline
\end{tabular}

\section{A. Taxonomies}

1) Management: Data management is related to storage, format and dimension of acquired data. The upper part of Fig. 12 shows the subcategories for each taxonomy.

Storage. The category defines the methodologies to keep and maintain the collected data. Two subcategories are defined, i.e., centralized and distributed, according to the location where data is made available.

Centralized. A MCS system operates a centralized storage management when data is stored and maintained in a single location, which is usually a database made available in the cloud. This approach is typically employed when significant processing or data aggregation is required. For instance, urban monitoring [140], price comparison [133] and emergency situation management [118] are domains that require a centralized storage.

Distributed. Storing data in a distributed manner is typically employed for delay-tolerant applications, i.e. when users are allowed to deliver data with a delayed reporting. For instance, when labeled data can be aggregated at the end of sensing campaign to map a phenomenon, such as air quality [56] and noise monitoring [115] as well as urban planning [162]. The recent fog computing and mobile/multi-access edge computing that make resources available close to the end users are also an example of distributed storage [278]. We will further discuss these paradigms in Sec. VIII-B.

Format. The class format divides data between structured and unstructured. Structured data is clearly defined, while unstructured data includes information that is not easy to find and requires significant processing because it comes with different formats.

Structured. Structured data has been created to be stored as a structure and analyzed. It is organized to let easy access and analysis, and typically is self-explanatory. Representative examples are identifiers and specific information, such as age and other characteristics of individuals.

Unstructured. Unstructured data does not have a specific identifier to be recognized by search functions easily. Representative examples are media files, such as video, audio, and images, and all types of files that require complex analysis, e.g., information 


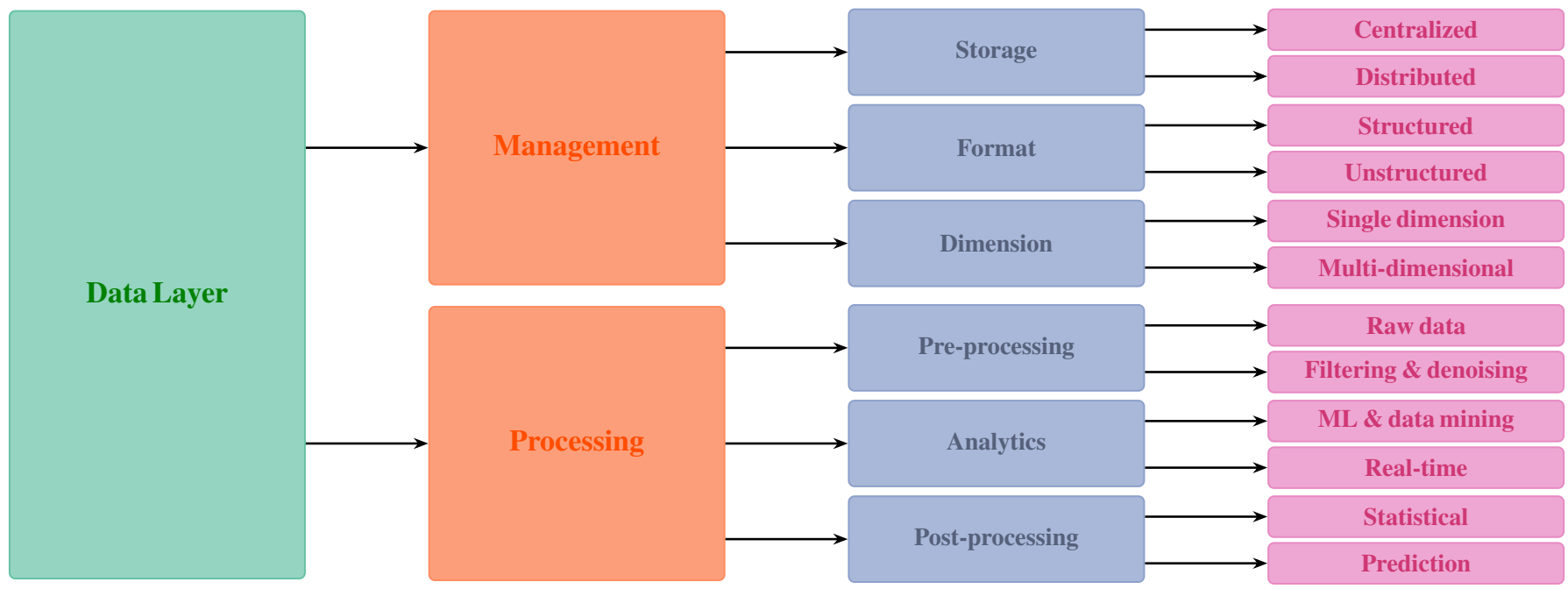

Fig. 12. Taxonomies on data layer, which includes management and processing categories. The management-related taxonomies are composed of storage, format, and dimension classes, while processing-related taxonomies are divided into pre-processing, analytics, and post-processing classes.

collected from social networks.

Dimension. Data dimension is related to the set of attributes of the collected data sets. For single dimension data, the attributes comprise a single type of data, and multi-dimensional when the data set includes more types of data.

Single dimension. Typically, applications exploiting a specific type of sensor produce single dimension data. Representative examples are environmental monitoring exploiting a dedicated sensor, e.g., air quality or temperature mapping.

Multi-dimensional. Typically, applications that require the use of different sensors or multimedia applications produce multidimensional data. For instance, mobile social networks are examples of multi-dimensional data sets because they allow users to upload video, images, audio, etc.

2) Processing: Processing data is a fundamental step in MCS campaigns. The lower part of Fig. 12 shows the classes of processing-related taxonomies, which are divided between pre-processing, analytics, and post-processing.

Pre-processing. Pre-processing operations are performed on collected data before analytics. We divide pre-processing into raw data and filtering, and denoising categories. Data is raw when no operations are executed before data analytics like in filtering and denoising.

Raw data. Raw data has not been treated by any manipulation operations. The advantage of storing raw data is that it is always possible to infer information applying different processing techniques at a later stage.

Filtering and denoising. Filtering and denoising refer to the main strategies employed to refine collected data by removing irrelevant and redundant data. In addition, they help to aggregate and make sense of data while reducing at the same time the volume to be stored.

Analytics. Data analytics aims to extract and expose meaningful information through a wide set of techniques. Corresponding taxonomies include machine learning (ML) \& data mining and real-time analytics.

$M L$ and data mining. ML and data mining category analyze data not in real-time. These techniques aim to infer information, identify patterns, or predict future trends. Typical applications that exploit these techniques in MCS systems are environmental monitoring and mapping, urban planning, and indoor navigation systems.

Real-time. Real-time analytics consists in examining collected data as soon as it is produced by the contributors. Analyzing data in real-time requires high responsiveness and computational resources from the system. Typical examples are campaigns for traffic monitoring, emergency situation management, or unmanned vehicles.

Post-processing. After data analytics, it is possible to adopt post-processing techniques for statistical reasons or predictive analysis.

Statistical. Statistical post-processing aims at inferring proportions given quantitative examples of the input data. For example, while mapping air quality, statistical methods can be applied to study sensed information, e.g., analyzing the correlation of rush hours and congested roads with air pollution by computing average values.

Prediction. Predictive post-processing techniques aim at determining future outcomes from a set of possibilities when given new input in the system. In the application domain of air quality monitoring, post-processing is a prediction when given a dataset of sensed data the organizer aims to forecast which will be the future air quality conditions (e.g., Wednesday at lunchtime).

\section{B. Classification}

In the following paragraphs, we survey literature works according to the taxonomy of data layer proposed above. 
CLASSIFICATION BASED ON MANAGEMENT TAXONOMY OF DATA LAYER

\begin{tabular}{|c|c|c|c|c|c|c|c|}
\hline \multirow[b]{2}{*}{ PROJECT } & \multirow[b]{2}{*}{ REFERENCE } & \multicolumn{2}{|c|}{ STORAGE } & \multicolumn{2}{|c|}{ FORMAT } & \multicolumn{2}{|c|}{ DIMENSION } \\
\hline & & Centralized & Distributed & Structured & Unstructured & Single dimension & Multi-dimensional \\
\hline HealthAware & [17] & & $\mathrm{x}$ & & $\mathrm{x}$ & & $\mathrm{x}$ \\
\hline DietSense & [19] & $\mathrm{x}$ & & & $\mathrm{x}$ & & $\mathrm{x}$ \\
\hline Nericell & [20] & $\mathrm{x}$ & & & $\mathrm{x}$ & & $\mathrm{x}$ \\
\hline NoiseMap & [115] & $\mathrm{x}$ & & $\mathrm{x}$ & & $\mathrm{x}$ & \\
\hline GasMobile & {$[21]$} & $\mathrm{x}$ & & $\mathrm{x}$ & & $\mathrm{x}$ & \\
\hline NoiseTube & [123] & $\mathrm{x}$ & & $\mathrm{x}$ & & $\mathrm{x}$ & \\
\hline CenceMe & [59] & & $\mathrm{x}$ & & $\mathrm{x}$ & & $\mathrm{x}$ \\
\hline MicroBlog & [60] & $\mathrm{x}$ & & & $\mathrm{x}$ & & $\mathrm{x}$ \\
\hline PEIR & [121] & $\mathrm{x}$ & & & $\mathrm{x}$ & & $\mathrm{x}$ \\
\hline How long to wait? & [142] & $\mathrm{x}$ & & & $\mathrm{x}$ & & $\mathrm{x}$ \\
\hline PetrolWatch & [131] & $\mathrm{x}$ & & $\mathrm{x}$ & & $\mathrm{x}$ & \\
\hline AndWellness & [136] & $\mathrm{x}$ & & & $\mathrm{x}$ & & $\mathrm{x}$ \\
\hline Darwin & [155] & & $\mathrm{x}$ & & $\mathrm{x}$ & & $\mathrm{x}$ \\
\hline CrowdSense@Place & [147] & $\mathrm{x}$ & & & $\mathrm{x}$ & & $\mathrm{x}$ \\
\hline ILR & [168] & & $\mathrm{x}$ & & $\mathrm{x}$ & & $\mathrm{x}$ \\
\hline SoundSense & [166] & & $\mathrm{x}$ & $\mathrm{x}$ & & $\mathrm{x}$ & \\
\hline Urban WiFi & [164] & & $\mathrm{x}$ & $\mathrm{x}$ & & $\mathrm{x}$ & \\
\hline LiveCompare & [133] & $\mathrm{x}$ & & $\mathrm{x}$ & & $\mathrm{x}$ & \\
\hline MobiClique & [150] & & $\mathrm{x}$ & & $\mathrm{x}$ & & $\mathrm{x}$ \\
\hline MobiShop & [132] & $\mathrm{x}$ & & $\mathrm{x}$ & & $\mathrm{x}$ & \\
\hline SPA & [134] & & $\mathrm{x}$ & & $\mathrm{x}$ & & $\mathrm{x}$ \\
\hline EmotionSense & [149] & & $\mathrm{x}$ & & $\mathrm{x}$ & & $\mathrm{x}$ \\
\hline ConferenceSense & [167] & & $\mathrm{x}$ & & $\mathrm{x}$ & & $\mathrm{x}$ \\
\hline Travel Packages & [169] & & $\mathrm{x}$ & & $\mathrm{x}$ & & $\mathrm{x}$ \\
\hline Mahali & [129] & $\mathrm{x}$ & & $\mathrm{x}$ & & & $\mathrm{x}$ \\
\hline Ear-Phone & [122] & $\mathrm{x}$ & & & $\mathrm{x}$ & $\mathrm{x}$ & \\
\hline WreckWatch & [143] & & $\mathrm{x}$ & $\mathrm{x}$ & & & $\mathrm{x}$ \\
\hline VTrack & [145] & $\mathrm{x}$ & & $\mathrm{x}$ & & & $\mathrm{x}$ \\
\hline Social Serendipity & [151] & & $\mathrm{x}$ & & $\mathrm{x}$ & & $\mathrm{x}$ \\
\hline SociableSense & [152] & & $\mathrm{x}$ & & $\mathrm{x}$ & & $\mathrm{x}$ \\
\hline WhozThat & [153] & & $\mathrm{x}$ & & $\mathrm{x}$ & & $\mathrm{x}$ \\
\hline $\mathrm{MoVi}$ & [154] & $\mathrm{x}$ & & & $\mathrm{x}$ & $\mathrm{x}$ & \\
\hline
\end{tabular}

Management. The research works are classified and surveyed in Table IX according to data management taxonomies discussed in V-A1.

Storage. The centralized strategy is typically used when the collector needs to have insights by inferring information from raw data or when data needs to be aggregated. To give representative examples, the centralized storage is exploited in monitoring noise [115], [123], air quality [21], [22], [56], traffic conditions [20], [142], prices of goods [131], [133]. MCS organizers exploit a distributed approach when they do not need to aggregate all gathered data or it is not possible for different constraints, such as privacy reasons. In order to address the integrity of the data and the contributors' privacy, the CenceMe application stores locally data to be processed by the phone classifiers and none of the raw collected data is sent to the backend [59]. For same reasons, applications related to healthcare [17], sharing travels [169], emotions [149], sounds [166], [167], or locations [164], [168] adopt a distributed local storage.

Format. Structured data is stored and analyzed as a single and self-explanatory structure, which is easy to be aggregated and compare between different contributions. For instance, samples that are aggregated together to map a phenomenon are structured data. Representative examples consist of environmental monitoring [21], [115], [122], [123], checking prices in real-time [131], [133], characterizing WiFi intensity [164]. Unstructured data cannot be compared and do not have a specific value, being characterized by multimedia, such as mobile social applications [150], [151], [155], comparing travel packages [169], improving location reliability [168], estimating bus arrival time [142], and monitoring health conditions [17], [136].

Dimension. Single dimension approach is typical of MCS systems that require data gathered from a specific sensor. Typical examples are monitoring phenomena that couple a detected value with its sensing location, such as noise [115], [122], [123] and air quality [21] monitoring, mapping WiFi intensity [164], and checking prices of goods [131], [150]. Multi-dimensional MCS campaigns aim to gather information from different types of sensors. Representative examples are mobile social networks [60], [152], [153], [155] and applications that consist in sharing multimedia, such as travelling [169], conferences [167], emotions [149].

Processing. Here we survey and classify works according the processing taxonomy presented in V-A2. Surveyed papers and their carachteristics are shown in Table X. 
TABLE X

CLASSIFICATION BASED ON PROCESSING TAXONOMY OF DATA LAYER

\begin{tabular}{|c|c|c|c|c|c|c|c|}
\hline \multirow[b]{2}{*}{ PROJECT } & \multirow[b]{2}{*}{ REFERENCE } & \multicolumn{2}{|c|}{ PRE-PROCESSING } & \multicolumn{2}{|c|}{ ANALYTICS } & \multicolumn{2}{|c|}{ POST-PROCESSING } \\
\hline & & Raw data & Filtering \& denoising & ML \& data mining & Real-time & Statistical & Prediction \\
\hline HealthAware & {$[17]$} & & $\mathrm{x}$ & & $\mathrm{x}$ & $\mathrm{x}$ & \\
\hline Nericell & {$[20]$} & & $\mathrm{x}$ & $\mathrm{x}$ & & $\mathrm{x}$ & \\
\hline NoiseMap & {$[115]$} & $\mathrm{x}$ & & & $\mathrm{x}$ & $\mathrm{x}$ & \\
\hline GasMobile & {$[21]$} & $\mathrm{x}$ & & $\mathrm{x}$ & & $\mathrm{x}$ & \\
\hline MicroBlog & {$[60]$} & & $\mathrm{x}$ & $\mathrm{x}$ & & $\mathrm{x}$ & \\
\hline PEIR & [121] & & $\mathrm{x}$ & $\mathrm{x}$ & & $\mathrm{x}$ & \\
\hline How long to wait? & [142] & & $\mathrm{x}$ & & $\mathrm{x}$ & & $\mathrm{x}$ \\
\hline PetrolWatch & [131] & & $\mathrm{x}$ & & $\mathrm{x}$ & $\mathrm{x}$ & \\
\hline AndWellness & {$[136]$} & & $\mathrm{x}$ & $\mathrm{x}$ & & $\mathrm{x}$ & \\
\hline Darwin & {$[155]$} & & $\mathrm{x}$ & $\mathrm{x}$ & & $\mathrm{x}$ & \\
\hline MobiClique & {$[150]$} & & $\mathrm{x}$ & $\mathrm{x}$ & & $\mathrm{x}$ & \\
\hline MobiShop & [132] & $\mathrm{x}$ & & $\mathrm{x}$ & & $\mathrm{x}$ & \\
\hline SPA & [134] & & $\mathrm{x}$ & $\mathrm{x}$ & & $\mathrm{x}$ & \\
\hline EmotionSense & [149] & & $\mathrm{x}$ & $\mathrm{x}$ & & $\mathrm{x}$ & \\
\hline ConferenceSense & {$[167]$} & & $\mathrm{x}$ & $\mathrm{x}$ & & $\mathrm{x}$ & \\
\hline Travel Packages & [169] & & $\mathrm{x}$ & $\mathrm{x}$ & & $\mathrm{x}$ & \\
\hline Mahali & [129] & & $\mathrm{x}$ & $\mathrm{x}$ & & $\mathrm{x}$ & \\
\hline Ear-Phone & [122] & $\mathrm{x}$ & & $\mathrm{x}$ & & $\mathrm{x}$ & \\
\hline WreckWatch & [143] & & $\mathrm{x}$ & & $\mathrm{x}$ & $\mathrm{x}$ & \\
\hline VTrack & [145] & & $\mathrm{x}$ & & $\mathrm{x}$ & & $\mathrm{x}$ \\
\hline Social Serendipity & [151] & & $\mathrm{x}$ & & $\mathrm{x}$ & $\mathrm{x}$ & \\
\hline SociableSense & {$[152]$} & & $\mathrm{x}$ & $\mathrm{x}$ & & $\mathrm{x}$ & \\
\hline
\end{tabular}

Pre-processing. It divides raw data and filtering \& denoising categories. Some applications collect raw data, without any further data pre-processing. In most cases, works that exploit raw data simply consider different types of collected data, such as associating a position taken from the GPS with a sensed value, such as $\mathrm{dB}$ for noise monitoring [115], [122], [123], a power for WiFi strength [164], a price for e-commerce [132], [133]. Filtering \& denoising indicates applications that perform operations on raw data. For instance, in health care applications is possible to recognize the activity or condition of a user from patterns collected from different sensors [17], such as accelerometer or gyroscope. Other phenomena in urban environments require more complex pre-processing operations, such as detecting traffic [20], [143].

Analytics. Most of MCS systems perform ML and data mining techniques after data is collected and aggregated. Typical applications exploiting this approach consist in mapping phenomena and resources in cities, such as WiFi intensity [164], air quality [21], [22], [56], noise [115], [123]. Other application domains employing this approach are mobile social networks [59], [60], [154] and sharing information, such as travel packages [169], food [116], sounds [166] and improving location reliability [168]. Darwin phones [155] performs ML techniques for privacy constraints, aiming to not send to the central collector personal data that are deleted after being analyzed in the mobile device, which sends only inferred information. Real-time analytics aim to infer useful information as soon as possible and require more computational resources. Consequently, MCS systems employ them only when needed by application domains, such as monitoring traffic conditions [20], waiting time of public transports [142], comparing prices [131], [133]. In WhozThat [153] is an exception of mobile social networks where real-time analytics is performed for contextaware sensing and detecting users in the surroundings. In health care, AndWellness [136] and SPA [134] have data mining analytics because it aims to provide advice from remote in a long-term perspective, while HealtAware [17] presents real-time analytics to check conditions mainly of elderly people.

Post-processing. Almost all of the considered works present statistical post-processing, where inferred information is analyzed with statistical methods and approaches. Environmental monitoring [21], [56], [115], [123], healthcare [17], [136], mobile social networks [59], [154], [155] are only a few examples that adopt statistical post-processing. Considering the works under analysis, the predictive approach is exploited only to predict the waiting time for bus arrival [142] and estimate 
traffic delays by VTrack [145]. Nonetheless, recent MCS systems have adopted predictive analytics in many different fields, such as unmanned vehicles and urban planning already discussed in Sec. III-B. In particular, urbanization issues require nowadays to develop novel techniques based on predictive analytics to improve historical experience-based decisions, such as where to open a new local business or how to manage mobility more smartly. We will further discuss these domains in Sec. VIII-B.

\section{TAXONOMIES AND CLASSIFICATION ON COMMUNICATION LAYER}

This Section presents the taxonomies on the communication layer, which is composed by technologies and reporting classes. The technologies taxonomy analyzes the types of network interfaces that can be used to report data. The reporting taxonomy analyzes different ways of reporting data, which are not related to technologies, but depends on the application domains and constraints of sensing campaigns. Fig. 13 shows subcategories of taxonomies on communication layer.

\section{A. Taxonomies}

1) Technologies: Infrastructured and infrastructure-less constitute the subcategories of the Technologies taxonomy, as shown in the upper part of Fig. 13. Infrastructured technologies are cellular or wireless local area network (WLAN), where the network relies on base stations or access points to establish communication links. In infrastructure-less technologies, proximity-based communications are enforced with peer-to-peer technologies such as LTE-Direct, WiFi-Direct, and Bluetooth.

Infrastructured. Infrastructured technologies indicate the need for an infrastructure for data delivery. In this class, we consider cellular data communications and WLAN interfaces. According to the design of each campaign, organizers can require a specific technology to report data or leave the choice to end users. Usually, cellular connectivity is used when a sensing campaign requires data delivery with bounds on latency that WiFi does not guarantee because of its unavailability in many places and contention-based techniques for channel access (e.g., building a real-time map for monitoring availability of parking slot [34]) On the other side, WLAN interfaces can be exploited for mapping phenomena that can tolerate delays and save costs to end users.

Cellular. Reporting data through cellular connectivity is typically required from sensing campaign that perform realtime monitoring and require data reception as soon as it is sensed. Representative areas where cellular networking should be employed are traffic monitoring, unmanned vehicles, and emergency situation management.

From a technological perspective, the required data rates and latencies that current LTE and 4G systems provide are sufficient for the purposes of MCS systems. The upcoming $5 \mathrm{G}$ revolution will, however, be relevant for MCS because of the following main reasons. First, 5G architecture is projected to be highly based on the concepts of network function virtualization and SDN. As a consequence, the core functionalities that now are performed by on custom-built hardware will run in distributed fashion leading to better mobility support. Second, the use of additional frequencies, for example in the millimeter-wave part of the spectrum, opens up the doors for higher data rates at the expense of high path-loss and susceptibility to blockages. This calls for much denser network deployments that make MCS systems benefit from better coverage. Additionally, services requiring higher data rates will be served by millimeter-wave base stations, making room for additional resources in the lower part of the spectrum where MCS services will be served.

WLAN. Typically, users tend to exploit WLAN interfaces to send data to the central collector for saving costs that cellular network impose with the subscription fees. The main drawback of this approach is the unavailability of WiFi connectivity. Consequently, this approach is used mainly when sensing organizers do not specify any preferred reporting technologies or when the application domain permits to send data also a certain amount of time after the sensing process. Representative domains that exploit this approach are environmental monitoring, and urban planning.

Infrastructure-less. It consists of device-to-device (D2D) communications that do not require any infrastructure as a network access point but rather allow devices in the vicinity to communicate directly.

WiFi-direct. WiFi Direct technology is one of the most popular D2D communication standard, which is also suitable in the context of MCS paradigm. A WiFi Direct Group is composed of a group owner (GO) and zero or more group members (GM) and a device can dynamically assume the role of GM or GO. In [279] the authors propose a system in which users report data to the central collector collaboratively. For each group, a GO is elected and it is then responsible for reporting aggregated information to the central collector through LTE interfaces.

LTE-direct. Among the emerging D2D communication technologies, LTE-Direct is a very suitable technology for the MCS systems. Compared to other D2D standards, LTE-Direct presents different characteristics that perfectly fit the potential of MCS paradigm. Specifically, it exhibits very low energy consumption to perform continuous scanning and fast discovery of mobile devices in proximity, and wide transmission range around 500 meters that allows to create groups with many participants [280].

Bluetooth. Bluetooth represents another energy-efficient strategy to report data through a collaboration between users [281]. In particular, Bluetooth Low Energy (BLE) is the employed low power version of standard Bluetooth specifically built for IoT environments, proposed in the Bluetooth 4.0 specification [282]. Its energy efficiency perfectly fits the usage of mobile devices, which require to work for long period with limited use of resources and low battery drain.

2) Reporting: The lower part of Fig. 13 shows taxonomies related to reporting, which are divided into upload mode, methodology, and timing classes. Unlike the previous category, reporting defines the ways in which the mobile devices report 


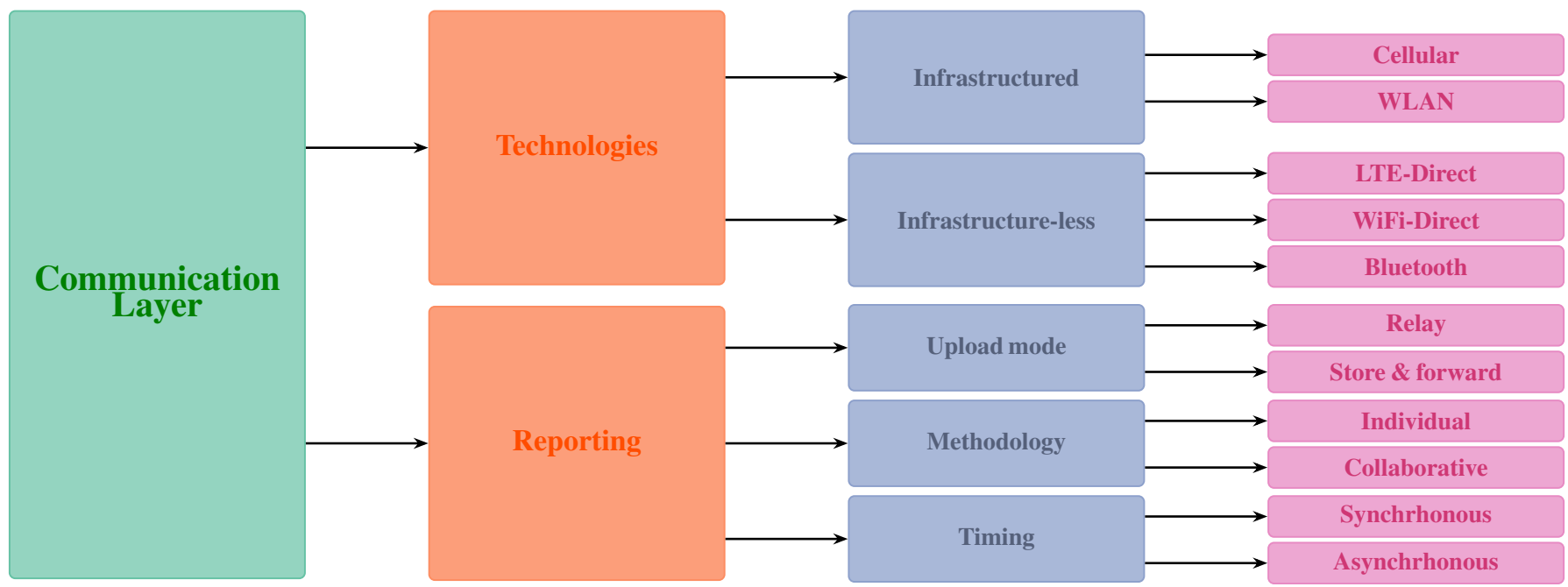

Fig. 13. Taxonomies on communication layer, which comprises technologies and reporting categories. The technologies-related taxonomies are composed of infrastructured and infrastructure-less classes, while reporting-related taxonomies are divided into upload mode, methodology, and timing classes.

sensed data to the central collector. Upload mode describes if data are delivered in real-time or in a delayed manner. The methodology category investigates how a mobile device executes the sensing process by itself and concerning other devices. Finally, timing analyzes if reporting between different contributors is synchronous or not.

Upload mode. Upload can be relay or store and forward, according to delay tolerance required by the application.

Relay. In this method, data is delivered as soon as collected. When mobile devices cannot meet real-time delivery, the best solution is to skip a few samples and in turns avoiding to waste energy for sensing or reporting, as the result may be inconsistent at the data collector [236]. Typical examples of time-sensitive tasks are emergency-related or monitoring of traffic conditions and sharing data with users through applications such as Waze [283].

Store and forward. This approach is typically used in delaytolerant applications when campaigns do not need to receive data in real-time. Data is typically labeled to include a reference to the sensed phenomenon [220]. For instance, traffic monitoring [142] or gluten sensors to share restaurants and food for people suffering celiac disease [116] are examples of sensing activities that do not present temporal constraints.

Methodology. This category considers how a device executes a sensing process in respect to others. Devices can act as peers and accomplish tasks in a collaborative way or individually and independently one from each other.

Individual. Sensing execution is individual when each user accomplishes the requested task individually and without interaction with other participants.

Collaborative. The collaborative approach indicates that users communicate with each other, exchange data and help themselves in accomplishing a task or delivering information to the central collector. Users are typically grouped and exchange data exploiting short-range communication technologies, such as WiFi-direct or Bluetooth [281]. Collaborative approaches can also be seen as a hierarchical data collection process, in which some users have more responsibilities than others. This paves the path for specific rewarding strategies to incentive users in being the owner of a group to compensate him for its higher energy costs.

Timing. Execution timing indicates if the devices should sense in the same period or not. This constraint is fundamental for applications that aim at comparing phenomena in a certain time window. Task reporting can be executed in synchronous or asynchronous fashion.

Synchronous. This category includes the cases in which users start and accomplish at the same time the sensing task. For synchronization purposes, participants can communicate with each other or receive timing information from a central authority. For instance, LiveCompare compares the live price of goods and users should start sensing synchronously. Otherwise, the comparison does not provide meaningful results [133]. Another example is traffic monitoring [20].

Asynchronous. The execution time is asynchronous when users perform sensing activity not in time synchronization with other users. This approach is particularly useful for environmental monitoring and mapping phenomena, receiving labeled data also in a different interval of time. Typical examples are mapping noise [115] and air pollution [21] in urban environments.

\section{B. Classification}

The following paragraphs classify MCS works according to the communication layer taxonomy previously described in this Section.

Technologies. Literature works are classified and surveyed in Table XI according to data management taxonomies discussed in VI-A1.

Infrastructured. An application may pretend a specific communication technology for some specific design requirement 
TABLE XI

CLASSIFICATION BASED ON TECHNOLOGIES TAXONOMY OF COMMUNICATION LAYER

\begin{tabular}{|c|c|c|c|c|c|c|}
\hline \multirow[b]{2}{*}{ PROJECT } & \multirow[b]{2}{*}{ REFERENCE } & \multicolumn{2}{|c|}{ INFRASTRUCTURED } & \multicolumn{3}{|c|}{ INFRASTRUCTURE-LESS } \\
\hline & & Cellular & WLAN & LTE-Direct & WiFi-Direct & Bluetooth \\
\hline HealthAware & [17] & & $\mathrm{x}$ & & & $\mathrm{x}$ \\
\hline DietSense & [19] & $\mathrm{x}$ & $\mathrm{x}$ & & & \\
\hline Nericell & [20] & $\mathrm{x}$ & & & & $\mathrm{x}$ \\
\hline NoiseMap & [115] & $\mathrm{x}$ & $\mathrm{x}$ & & & \\
\hline GasMobile & [21] & $\mathrm{x}$ & $\mathrm{x}$ & & & \\
\hline NoiseTube & [123] & $\mathrm{x}$ & $\mathrm{x}$ & & & \\
\hline CenceMe & [59] & $\mathrm{x}$ & $\mathrm{x}$ & & & \\
\hline MicroBlog & {$[60]$} & $\mathrm{x}$ & $\mathrm{x}$ & & & \\
\hline PEIR & [121] & $\mathrm{x}$ & $\mathrm{x}$ & & & $\mathrm{x}$ \\
\hline How long to wait? & [142] & $\mathrm{x}$ & & & & \\
\hline PetrolWatch & [131] & $\mathrm{x}$ & & & & \\
\hline AndWellness & [136] & $\mathrm{x}$ & $\mathrm{x}$ & & & \\
\hline Darwin & [155] & $\mathrm{x}$ & $\mathrm{x}$ & & & $\mathrm{x}$ \\
\hline CrowdSense@Place & [147] & & $\mathrm{x}$ & & & \\
\hline ILR & [168] & $\mathrm{x}$ & $\mathrm{x}$ & & & $\mathrm{x}$ \\
\hline SoundSense & [166] & $\mathrm{x}$ & $\mathrm{x}$ & & & \\
\hline Urban WiFi & [164] & & $\mathrm{x}$ & & & \\
\hline LiveCompare & [133] & $\mathrm{x}$ & $\mathrm{x}$ & & & \\
\hline MobiClique & {$[150]$} & $\mathrm{x}$ & $\mathrm{x}$ & & & $\mathrm{x}$ \\
\hline MobiShop & [132] & $\mathrm{x}$ & $\mathrm{x}$ & & & \\
\hline SPA & [134] & & $\mathrm{x}$ & & & $\mathrm{x}$ \\
\hline EmotionSense & [149] & $\mathrm{x}$ & $\mathrm{x}$ & & & $\mathrm{x}$ \\
\hline ConferenceSense & [167] & & $\mathrm{x}$ & & & $\mathrm{x}$ \\
\hline Travel Packages & [169] & $\mathrm{x}$ & $\mathrm{x}$ & & & \\
\hline Mahali & [129] & $\mathrm{x}$ & $\mathrm{x}$ & & & \\
\hline Ear-Phone & [122] & $\mathrm{x}$ & $\mathrm{x}$ & & & \\
\hline WreckWatch & [143] & $\mathrm{x}$ & & & & \\
\hline VTrack & [145] & $\mathrm{x}$ & $\mathrm{x}$ & & & \\
\hline Social Serendipity & [151] & $\mathrm{x}$ & $\mathrm{x}$ & & & $\mathrm{x}$ \\
\hline SociableSense & [152] & $\mathrm{x}$ & $\mathrm{x}$ & & & $\mathrm{x}$ \\
\hline WhozThat & [153] & $\mathrm{x}$ & $\mathrm{x}$ & & & $\mathrm{x}$ \\
\hline $\mathrm{MoVi}$ & [154] & $\mathrm{x}$ & $\mathrm{x}$ & & & \\
\hline
\end{tabular}

Note that the set of selected works was developed much before the definition of the standards LTE-Direct and WiFi-Direct, thus these columns have no corresponding marks.

or leave to the users the choice of which type of transmission exploit. Most of the considered applications do not specify a required communication technology to deliver data. Indeed, the Table presents corresponding marks to both WLAN and cellular data connectivity. Applications that permit users to send data as preferred aim to collect as much data as possible without having specific design constraints, such as mobile social networks [59], [153]-[155], mapping noise [115], [122], [123] or air pollution [21], [22], or monitoring health conditions [19], [136]. Other examples consist in applications that imply a participatory approach that involves users directly and permit them to choose how to deliver data, such as sharing information on environment [121], sounds [166], emotions [149], travels [169], and space weather monitoring [129]. Some applications require a specific communication technology to deliver data for many different reasons. On the one side, MCS systems that require data cellular connectivity typically aim to guarantee a certain spatial coverage or real-time reporting that WiFi availability cannot guarantee. Some representative examples are monitoring traffic conditions [20], [143], predicting bus arrival time [142], and comparing fuel prices [131]. On the other side, applications that require transmission through WLAN interfaces usually do not have constraints on spatial coverage and real-time data delivery, but aim to save costs to users (e.g., energy and data plan consumptions). For instance, Crowdsense@Place [147],
SPA [134], and HealthAware [17] transmit only when a WiFi connection is available and mobile devices are line-powered to minimize energy consumption.

Infrastructure-less. Recently, MCS systems are increasing the usage of D2D communication technologies to exchange data between users in proximity. While most important MCS works under analysis for our taxonomies and corresponding classification have been developed before the definition of $\mathrm{WiFi}_{-}$ Direct and LTE-Direct and none of them utilize these standards, some of them employ Bluetooth, as shown in Table XI. For instance, Bluetooth is used between users in proximity for social networks purposes [151], [153], [155], environmental monitoring [121]. and exchanging information [149], [167].

Reporting. Literature works are classified and surveyed in Table XII according to data management taxonomies discussed in VI-A2.

Upload mode. Upload can can be divided between relay or store \& forward according to the application requirements. Relay is typically employed for real-time monitoring, such as traffic conditions [20], [142], [143], [145], or price comparison [132], [133]. Store \& forward approach is used in applications without strict time constraints that are typically related to labeled data. For instance, mapping phenomena in a city like noise [122], [123], air quality [21], or characterizing 
TABLE XII

CLASSIFICATION BASED ON REPORTING TAXONOMY OF COMMUNICATION LAYER

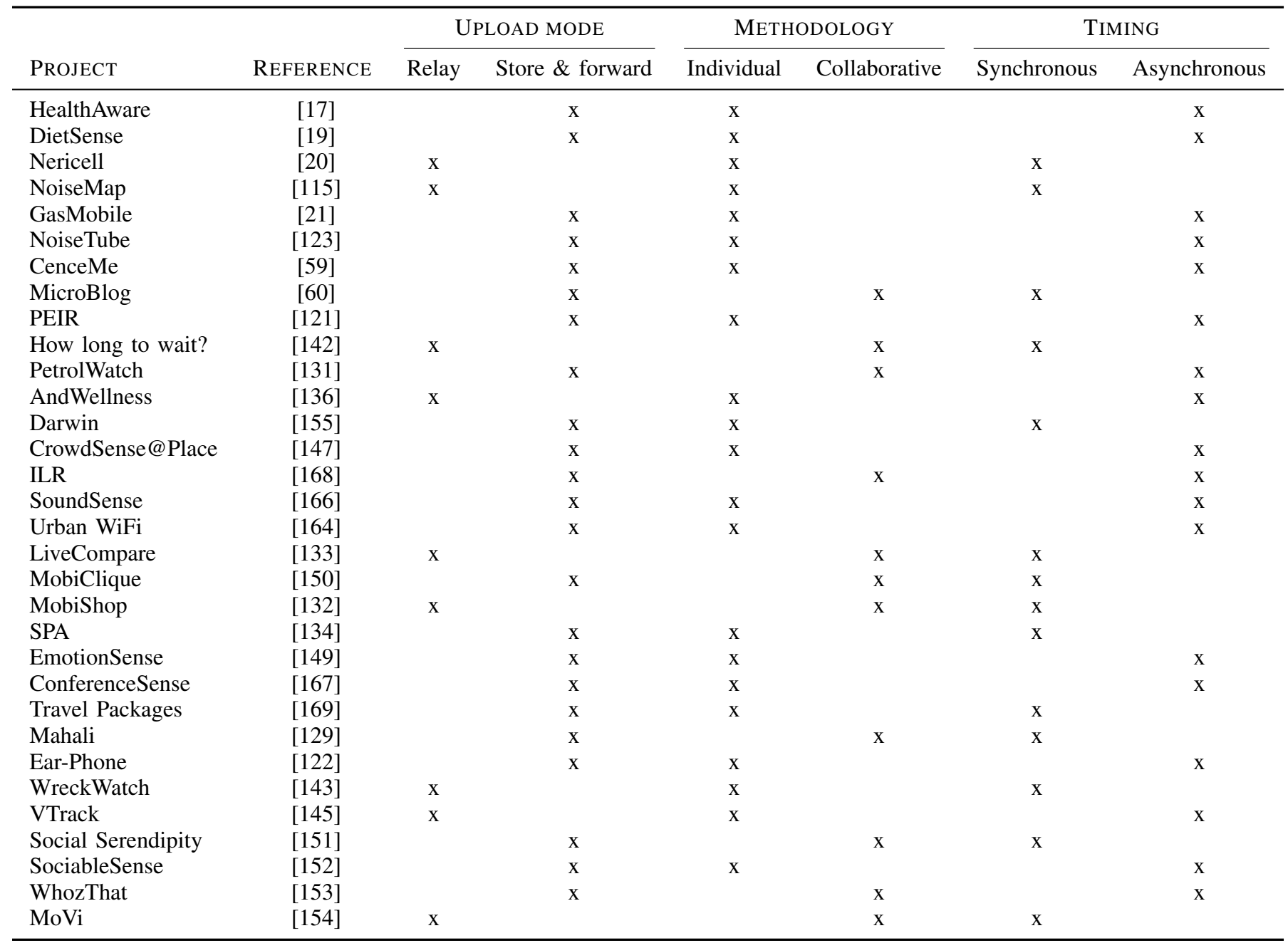

WiFi coverage [164] can be reported also at the end of the to save energy. Other applications are not so tolerant, but still do not require strict constraints, such as mobile social networks [59], [60], [150]-[153], [155], health care [17], [19], price comparison [131], reporting info about environment [121], sounds [166], conferences [167], or sharing and recommending travels [169]. Despite being in domains that usually are delay tolerant, NoiseMap [115] and AndWellness [136] are applications that provide real-time services and require the relay approach.

Methodology. It is divided between individual and collaborative MCS systems. Most of MCS systems are based on individual sensing and reporting without collaboration between the mobile devices. Typical examples are healthcare applications [17], [19], noise [115], [122], [123] [136]. Note that systems that create maps merging data from different users are considered individual because users do not interact between each other to contribute. Some examples are air quality [21], [22], [56] and noise monitoring [115], traffic estimation [20], [143], [145]. Mobile social networks are typical collaborative systems, being characterized by sharing and querying actions that require interactions between users [60], [62], [151], [153], [155]. To illustrate, monitoring prices in e-commerce [132], [133] and the bus arrival time [142] are other examples of user collaborating to reach the scope of the application.

Timing. This classification specifies if the process of sensing needs users contributing synchronously or not. The synchronous approach is typically requested by applications that aim at comparing services in real-time, such as prices of goods [132], [133]. Monitoring and mapping noise pollution is an example of application that can be done with both synchronous or asynchronous approach. For instance, [115] requires a synchronous design because all users perform the sensing at the same time. Typically this approach aims to infer data and have the results while the sensing process is ongoing, such as monitoring traffic condition is useful only if performed by users at the same time [20]. Asynchronous MCS system do not require simultaneous users' contribution. Mapping WiFi signal strength intensity [164] or noise pollution [123] [122] are typically performed with this approach. Healthcare applications do not require contemporary contributions [17]. Some mobile social networks do not require users to share their interests at the same time [152], [153]. 


\section{TAXONOMIES AND ClaSSIFICATION ON SENSING LAYER}

This Section discusses the taxonomies of the sensing layer A general representation of the sensing layer is presented in Fig. 8. The taxonomy is composed by elements and sampling process categories, that are respectively discussed in Subsections VII-A1 and VII-A2. Then, Subsection VII-B classifies the research works according to the proposed taxonomies. This Section does not delve into the technicalities of sensors as there is a vast literature on regard. We refer the interested reader to surveys on smartphone sensors [48], [49] and mobile phone sensing [8], [9].

\section{A. Taxonomies}

1) Elements: As shown in the upper part of Fig. 14, sensing elements can be mainly classified into three characteristics according to their deployment, activity, and acquisition. In the deployment category, we distinguish between the dedicated and non-dedicated deployment of sensors in the mobile devices. The activity differentiates between sensors that are always-on because they are assigned basic operations of the device and those that require user intervention to become active. Finally, the acquisition category indicates if the type of collected data is homogeneous or heterogeneous.

Deployment. The majority of available sensors are built-in and embedded in mobile devices. Nevertheless, non-embedded sensing elements exist and are designed for very specific purposes. For this type of sensing equipment, vendors typically do not have an interest in large scale production. For example, the gluten sensor comes as a standalone device and it is designed to work in couple with smartphones that receive, store, and process food records of gluten detection [116]. Therefore it becomes necessary to distinguish between popular sensors typically embedded in mobile devices and specific ones that are mostly standalone and connected to the device. As mentioned in [284], sensors can be deployed either in dedicated or nondedicated forms. The latter definition includes sensors that are employed for multiple purposes, while dedicated sensors are typically designed for a specific task.

Non-dedicated. Integrating non-dedicated sensors into mobile devices is nowadays common practice. Sensors are essential for ordinary operations of smartphones (e.g., the microphone for phone calls), for social purposes (e.g., the camera to take pictures and record videos) and for user applications (e.g., GPS for navigation systems). These sensors do not require to be paired with other devices for data delivery but exploit the communication capabilities of mobile devices.

Dedicated. These sensors are typically standalone devices dedicated to a specific purpose and are designed to be paired with a smartphone for data transmission. Indeed, they are very small devices with limited storage capabilities. Communications rely on wireless technologies like Bluetooth or Near Field Communications (NFC). Nevertheless, specific sensors such as the GasMobile hardware architecture can be wired connected with smartphones through USBs [21]. Whereas non-dedicated sensors are used only for popular applications, the design of dedicated sensors is very specific and either single individuals or the entire community can profit. To illustrate, only the celiac community can take advantage of the gluten sensor [116] while an entire city can benefit from the fine dust sensors [127] or nuclear radiation monitoring [120].

Activity. Nowadays mobile devices require a growing number of basic sensors to operate that provide basic functionalities and cannot be switched off. For example, auto-adjusting the brightness of the screen requires the ambient light sensor being active while understanding the orientation of the device can be possible only having accelerometer and gyroscope working continuously. Conversely, several other sensors can be switched on and off manually by user intervention: taking a picture or recording a video requires the camera being active only for a while. We divide these sensors between always-on and on demand sensors. This classification unveils a number of properties. For example, always-on sensors perform sensing continuously and they operate consuming a small amount of energy. Having such deep understanding helps devising applications using sensor resources properly, such as exploiting an always-on and low consuming sensor to switch on or off another one. For instance, turning off the screen using the proximity sensor helps saving battery lifetime as the screen is a major cause of energy consumption [285]. Turning off the ambient light sensor and the GPS when the user is not moving or indoor permits additional power savings.

Always-on. These sensors are required to accomplish mobile devices basic functionalities, such as detection of rotation and acceleration. As they run continuously and consume a minimal amount of energy, it has become convenient for several applications to make use of these sensors in also in different contexts. Activity recognition such as detection of movement patterns (e.g. walking/running [286]-[288]) or actions (e.g. driving, riding a car or sitting [289]-[291]) is a very important feature that the accelerometers enable. To recognize user activities, some works also exploit readings from pressure sensor [83]. Furthermore, if used in pair with the gyroscope, sensing readings from the accelerometers enable to monitor the user driving style [80]. Also, some applications use these sensors for context-awareness and energy savings, detecting user surroundings and disabling not needed sensors (e.g., GPS in indoor environments) [292].

On demand. On demand sensors need to be switched on by users or exploiting an application running in background. Typically, they serve more complex applications than alwayson sensors and consume a higher amount of energy. Hence, it is better to use them only when they are needed. As a consequence, they consume much more energy and for this reason they are typically disabled for power savings. Typical representative examples are the camera for taking a picture, the microphone to reveal the level of noise in $\mathrm{dB}$ and the GPS to sense the exact position of a mobile device while sensing something (e.g., petrol prices in a gas station located anywhere [131]).

Acquisition. When organizers design a sensing campaign, implicitly consider which is the data necessary and in turns 


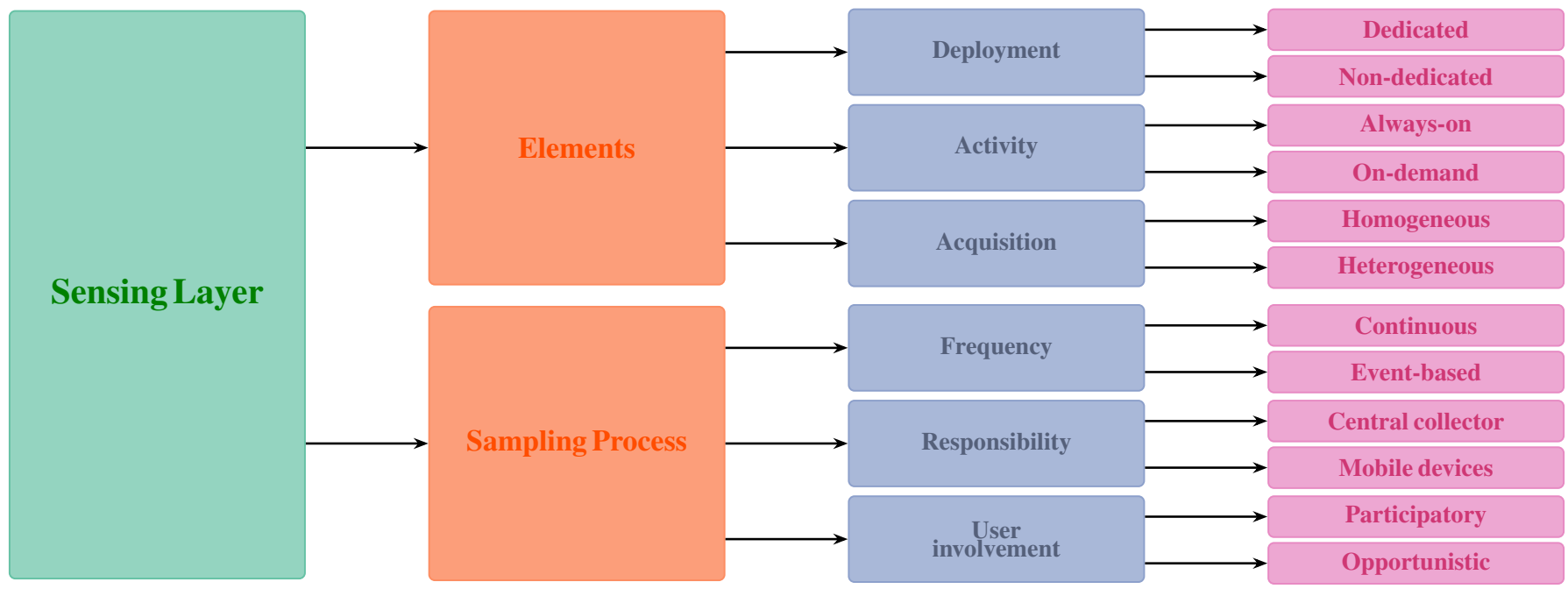

Fig. 14. Taxonomies on sensing layer, which comprises elements and sampling process categories. The elements-related taxonomies are divided into deployment, activity, and acquisition classes, while sampling-related taxonomies are composed of frequency, responsibility, and user involvement classes.

the required sensors. This category identifies if the acquisition provides homogeneous data or heterogeneous. Different sensors generate different types of data, often non-homogeneous. For instance, a microphone can be used to record an audio file or to sense the level of noise measured in $\mathrm{dB}$.

Homogeneous. We classify as homogeneous a data acquisition when it involves only one type of data and it does not change from one user to another one. For instance, air quality monitoring [128] is a typical example of this category because all the users sense the same data using dedicated sensors connected to their mobile devices.

Heterogeneous. Data acquisition is heterogeneous when it involves different data types usually sampled from several sensors. Typical examples include all the applications that employ a thread running on the background and infer information processing data after the sensing.

2) Sampling Process: The sampling process category investigates the decision-making process and is broadly subdivided in frequency, responsibility, and user involvement. The lower part of Fig. 14 shows the taxonomies of this category. In frequency we consider the sampling decision, which is divided between continuous or event-based sensing. The category responsibility focuses on the entity that is responsible for deciding to sense or not. The class user involvement analyzes if the decision of sampling is taken by the users actively or with an application running in the background.

Frequency. It indicates the frequency of sensing. In this category, we analyze how often a task must be executed. Some types of tasks can be triggered by event occurrence or because the device is in a particular context. On the other hand, some tasks need to be performed continuously.

Continuous. A continuous sensing indicates tasks that are accomplished regularly and independently by the context of the smartphone or the user activities. As once the sensors involved in the sensing process are activated, they provide readings at a given sampling rate. The data collection continues until there is a stop from the central collector (e.g., the quantity of data is enough) or from the user (e.g., when the battery level is low). In continuous sensing it is very important to set a sampling period that should be neither too low nor too high, to result in a good choice for data accuracy and in the meanwhile not too energy consuming. For instance, air pollution monitoring [56] must be based on continuous sensing to have relevant results and should be independent of some particular event.

Event-based. The frequency of sensing execution is event-based when data collection starts after a certain event has occurred. In this context, an event can be seen as an active action from a user or the central collector, but also a given context awareness (e.g., users moving outdoor, or getting on a bus). As a result, the decision-making process is not regular, but it requires an event to trigger the process. When users are aware of their context and directly perform the sensing task, typical examples are to detect food allergies [116] and to take pictures after a car accident or a natural disaster like an earthquake. When a user is not directly involved in the sensing, tasks can be event-based upon recognition of the context (e.g., user getting on a bus [142]).

Responsibility. It defines the entity that is responsible for taking the decision of sensing. On the one hand, mobile devices can take proper decisions following a distributed paradigm. On the other hand, the central collector can be designed to be responsible for taking sensing decisions in a centralized fashion. The collector has a centralized view of the amount of information already collected and therefore can distribute tasks among users or demand for data in a more efficient manner.

Mobile devices. Devices or users take sampling decisions locally and independently from the central authority. The single individual decides actively when, where, how, and what to sample, e.g., taking a picture for sharing the costs of goods [132]. When devices take sampling decisions, it is often necessary to detect the context in which smartphones and wearable devices are. The objective is to maximize the 
utility of data collection and minimizing the cost of performing unnecessary operations.

Central collector. In the centralized approach, the collector takes decisions about sensing and communicate them to the mobile devices. Centralized decisions can fit both participatory and opportunistic paradigms. If the requests are very specific, they can be seen as tasks by all means and indeed the centralized decision paradigm suits very well a direct involvement of users in sensing. However, if the requests are not very specific, but contain generic information, a mobile application running in the background is exploited.

User involvement. In MCS literature, user involvement is a very generic concept that can assume different meanings according to the context in which it is considered. In addition, participatory sensing is often used only to indicate that sensing is performed by participants [58]. We use the term user involvement to define if a sensing process requires or not active actions from the device's owner. We classify as opportunistic the approach in which users are not directly involved in the process of gathering data and usually an application is run in background (e.g., sampling user movements from the GPS) The opposite approach is the participatory one, which requires an active action from users to gather information (e.g., taking a picture of a car accident). In the following, we discuss both approaches in details giving practical examples.

Participatory approach. It requires active actions from users, who are explicitly asked to perform specific tasks. They are responsible to consciously meet the application requests by deciding when, what, where, and how to perform sensing tasks (e.g., to take some pictures with the camera due to environmental monitoring, or record audio due to noise analysis). Upon the sensing tasks are submitted by the MCS platform, the users take an active part in the task allocation process as manually decide to accept or decline an incoming sensing task request [10]. In comparison to the opportunistic approach, complex operations can be supported by exploiting human intelligence who can solve the problem of context awareness in a very efficient way. Multimedia data can be crowdsensed in a participatory manner, such as images for price comparison [133] or audio signals for noise analysis [123]) [58]. Participation is at the users' discretion while the users are to be rewarded on the basis of their level of participation, as well as the quality of the data they provide [11]. In participatory sensing, making sensing decisions is only under the user's discretion; hence context-awareness is not a critical component, as opposed to the opportunistic sensing [51]. As data accuracy aims at minimum estimation error when crowdsensed data is aggregated to sense a phenomenon, participatory sensing can avoid extremely low accuracies by human intervention [35]. A grand challenge in MCS occurs due to the battery limitation of mobile devices. When users are recruited in a participatory manner, the monitoring and control of battery consumption are also delegated from the MCS platform to the users, who are responsible for avoiding transmitting low-quality data.

Opportunistic approach. In this approach users do not have direct involvement, but only declare their interest in joining a campaign and providing their sensors as a service. Upon a simple handshake mechanism between the user and the MCS platform, a MCS thread is generated on the mobile device (e.g., in the form of a mobile app), and the decisions of what, where, when, and how to perform the sensing are delegated to the corresponding thread. After having accepted the sensing process, the user is totally unconscious with no tasks to perform and data collection is fully automated. Either the MCS platform itself or the background thread that communicates with the MCS platform follows a decisionmaking procedure to meet a pre-defined objective function [37]. The smartphone itself is context-aware and makes decisions to sense and store data, automatically determining when its context matches the requirements of an application. Therefore, coupling opportunistic MCS systems with context-awareness is a crucial requirement. Hence, context awareness serves as a tool to run predictions before transmitting sensed data or even before making sensing decisions. For instance, in the case of multimedia data (e.g., images, video, etc.), it is not viable to receive sensing services in an opportunistic manner. However, it is possible to detect road conditions via accelerometer and GPS readings without providing explicit notification to the user [293]. As opposed to the participatory approach, the MCS platform can distribute the tasks dynamically by communicating with the background thread on the mobile device and by considering various criteria [180], [209], [210]. As opportunistic sensing completely decouples the user and MCS platform, as a result of the lack of user pre-screening mechanism on the quality of certain types of data (e.g. images, video, etc.), energy consumption might be higher since even low-quality data will be transmitted to the MCS platform even if it will be eventually discarded [294]. Moreover, the thread that is responsible for sensing tasks continues sampling and draining the battery. In order to avoid such circumstances, energy-aware MCS strategies have been proposed [295], [296].

\section{B. Classification}

This section surveys literature works according to the sensing layer taxonomy previously proposed.

Elements. It classifies the literature works according to the taxonomy presented in VII-A1. The characteristics of each paper are divided between the subcategories shown in Table XIII.

Deployment. Non-dedicated sensors are embedded in smartphones and typically exploited for context awareness. Accelerometer can be used for pattern recognition in healthcare applications [17], [136], road and traffic conditions [20], [142], [145]. The microphone can be useful for mapping the noise pollution in urban environments [115], [122], [123] or to recognize sound patterns [166]. In some applications, using specialized sensors requires a direct involvement of users. For instance, E-commerce exploits the combination between camera and GPS [131]-[133] while mobile social networks utilize most popular built-in sensors [60], [149], [150], [153], [155]. Specialized sensing campaigns employ dedicated sensors, which are typically not embedded in mobile devices and require to be connected to them. The most popular applications that deploy dedicated sensors are environmental monitoring, such as air quality [21], [56], nuclear radiations [120], space 
TABLE XIII

CLASSIFICATION BASED ON ELEMENTS TAXONOMY OF SENSING LAYER

\begin{tabular}{|c|c|c|c|c|c|c|c|}
\hline \multirow[b]{2}{*}{ PROJECT } & \multirow[b]{2}{*}{ REFERENCE } & \multicolumn{2}{|c|}{ DEPLOYMENT } & \multicolumn{2}{|c|}{ ACTIVITY } & \multicolumn{2}{|c|}{ ACQUISITION } \\
\hline & & Dedicated & Non-dedicated & Always-on & On demand & Homogeneous & Heterogeneous \\
\hline HealthAware & [17] & & $\mathrm{x}$ & & $\mathrm{x}$ & & $\mathrm{x}$ \\
\hline DietSense & [19] & & $\mathrm{x}$ & & $\mathrm{x}$ & & $\mathrm{x}$ \\
\hline Nericell & [20] & & $\mathrm{x}$ & $\mathrm{x}$ & & $\mathrm{x}$ & \\
\hline NoiseMap & [115] & & $\mathrm{x}$ & $\mathrm{x}$ & & & $\mathrm{x}$ \\
\hline GasMobile & {$[21]$} & $\mathrm{x}$ & & & $\mathrm{x}$ & $\mathrm{x}$ & \\
\hline NoiseTube & [123] & & $\mathrm{x}$ & & $\mathrm{x}$ & $\mathrm{x}$ & \\
\hline CenceMe & [59] & & $\mathrm{x}$ & & $\mathrm{x}$ & & $\mathrm{x}$ \\
\hline MicroBlog & {$[60]$} & & $\mathrm{x}$ & & $\mathrm{x}$ & & $\mathrm{x}$ \\
\hline PEIR & [121] & & $\mathrm{x}$ & $\mathrm{x}$ & & & $\mathrm{x}$ \\
\hline How long to wait? & [142] & & $\mathrm{x}$ & & $\mathrm{x}$ & & $\mathrm{x}$ \\
\hline PetrolWatch & [131] & & $\mathrm{x}$ & & $\mathrm{x}$ & $\mathrm{x}$ & \\
\hline AndWellness & [136] & & $\mathrm{x}$ & & $\mathrm{x}$ & & $\mathrm{x}$ \\
\hline Darwin & {$[155]$} & & $\mathrm{x}$ & & $\mathrm{x}$ & & $\mathrm{x}$ \\
\hline CrowdSense@Place & [147] & & $\mathrm{x}$ & $\mathrm{x}$ & & & $\mathrm{x}$ \\
\hline ILR & [168] & & $\mathrm{x}$ & $\mathrm{x}$ & & $\mathrm{x}$ & \\
\hline SoundSense & [166] & & $\mathrm{x}$ & & $\mathrm{x}$ & & $\mathrm{x}$ \\
\hline Urban WiFi & [164] & & $\mathrm{x}$ & $\mathrm{x}$ & & $\mathrm{x}$ & \\
\hline LiveCompare & [133] & & $\mathrm{x}$ & & $\mathrm{x}$ & & $\mathrm{x}$ \\
\hline MobiClique & {$[150]$} & & $\mathrm{x}$ & & $\mathrm{x}$ & & $\mathrm{x}$ \\
\hline MobiShop & [132] & & $\mathrm{x}$ & & $\mathrm{x}$ & $\mathrm{x}$ & \\
\hline SPA & [134] & $\mathrm{x}$ & & $\mathrm{x}$ & & & $\mathrm{x}$ \\
\hline EmotionSense & [149] & & $\mathrm{x}$ & $\mathrm{x}$ & & & $\mathrm{x}$ \\
\hline ConferenceSense & {$[167]$} & & $\mathrm{x}$ & & $\mathrm{x}$ & $\mathrm{x}$ & \\
\hline Travel Packages & [169] & & $\mathrm{x}$ & & $\mathrm{x}$ & & $\mathrm{x}$ \\
\hline Mahali & [129] & $\mathrm{x}$ & & $\mathrm{x}$ & & & $\mathrm{x}$ \\
\hline Ear-Phone & {$[122]$} & & $\mathrm{x}$ & $\mathrm{x}$ & & $\mathrm{x}$ & \\
\hline WreckWatch & [143] & & $\mathrm{x}$ & $\mathrm{x}$ & & & $\mathrm{x}$ \\
\hline VTrack & [145] & & $\mathrm{x}$ & $\mathrm{x}$ & & & $\mathrm{x}$ \\
\hline Social Serendipity & {$[151]$} & $\mathrm{x}$ & & $\mathrm{x}$ & & & $\mathrm{x}$ \\
\hline SociableSense & [152] & & $\mathrm{x}$ & & $\mathrm{x}$ & & $\mathrm{x}$ \\
\hline WhozThat & [153] & & $\mathrm{x}$ & & $\mathrm{x}$ & & $\mathrm{x}$ \\
\hline $\mathrm{MoVi}$ & [154] & & $\mathrm{x}$ & $\mathrm{x}$ & & $\mathrm{x}$ & \\
\hline
\end{tabular}

weather [129], and emergencies management like floodings [24]. Other MCS systems that exploit dedicated sensors are in health care [134], e.g., to check the quantity of gluten in food [116] for celiacs.

Activity. For everyday usage mobile devices leverage on alwayson and low consuming sensors, which are also employed by many different applications. For instance, accelerometer is used to detect road conditions [20] and microphone for noise pollution [115], [121], [122], [129]. Typical examples using on-demand sensors are mobile social networks [60], [150], [153], [155], e-commerce [132], [133], and wellbeing applications [17], [19], [136].

Acquisition. Homogeneous acquisition includes MCS systems that collect data of one type. For instance, comparing prices of goods or fuel requires only the info of the price, which can be collected through an image or bar code [131], [132]. Noise and air monitoring are other typical examples that sample respectively a value in $\mathrm{dB}$ [122], [123] and measure of air pollution [21]. Movi [154] consists in contributing video captured from the camera through collaborative sensing. Some applications on road monitoring are also homogeneous, for instance to monitor traffic conditions [20]. Other applications exploiting only a type of data can be mapping WiFi signal [164] or magnetometer [297] for localization, enhancing location reliability [168] or sound patterns [167]. Most of MCS campaigns require multiple sensors and a heterogeneous acquisition. Typical applications that exploit multiple sensors are mobile social networks [60], [150], [151], [153], [155]. Health care applications typically require interaction between different sensors, such as accelerometer to recognize movement patterns and specialized sensors [17], [19], [136]. For localization, both GPS and WiFi signals can be used [298]. Intelligent transportation systems usually require the use of multiple sensors, including predicting bus arrival time [142], monitoring the traffic condition [145].

Sampling. Sampling category surveys and classifies works according to the taxonomy presented in VII-A2. The characteristics of each paper divided between the subcategories are shown in Table XIV.

Frequency. A task is performed continuously when it has temporal and spatial constraints. For instance, monitoring noise [115], [122], [123], air pollution [21], road and traffic conditions [20], [145] require ideally to collect information continuously to receive as much data as possible in the whole 
TABLE XIV

CLASSIFICATION BASED ON SAMPLING TAXONOMY OF SENSING LAYER

\begin{tabular}{|c|c|c|c|c|c|c|c|}
\hline \multirow[b]{2}{*}{ PROJECT } & \multirow[b]{2}{*}{ REFERENCE } & \multicolumn{2}{|c|}{ FREQUENCY } & \multicolumn{2}{|c|}{ RESPONSIBILITY } & \multicolumn{2}{|c|}{ USER INVOLVEMENT } \\
\hline & & Continuous & Event-based & Local & Centralized & Participatory & Opportunistic \\
\hline HealthAware & [17] & & $\mathrm{x}$ & $\mathrm{x}$ & & $\mathrm{x}$ & \\
\hline DietSense & [19] & & $\mathrm{x}$ & $\mathrm{x}$ & & $\mathrm{x}$ & \\
\hline Nericell & [20] & $\mathrm{x}$ & & $\mathrm{x}$ & & & $\mathrm{x}$ \\
\hline NoiseMap & [115] & $\mathrm{x}$ & & $\mathrm{x}$ & & $\mathrm{x}$ & \\
\hline GasMobile & {$[21]$} & $\mathrm{x}$ & & $\mathrm{x}$ & & $\mathrm{x}$ & \\
\hline NoiseTube & [123] & $\mathrm{x}$ & & $\mathrm{x}$ & & $\mathrm{x}$ & \\
\hline CenceMe & [59] & & $\mathrm{x}$ & $\mathrm{x}$ & & $\mathrm{x}$ & \\
\hline MicroBlog & {$[60]$} & & $\mathrm{x}$ & $\mathrm{x}$ & & $\mathrm{x}$ & \\
\hline PEIR & [121] & $\mathrm{x}$ & & & $\mathrm{x}$ & $\mathrm{x}$ & \\
\hline How long to wait? & [142] & & $\mathrm{x}$ & $\mathrm{x}$ & & & $\mathrm{x}$ \\
\hline PetrolWatch & [131] & & $\mathrm{x}$ & $\mathrm{x}$ & & & $\mathrm{x}$ \\
\hline AndWellness & [136] & & $\mathrm{x}$ & $\mathrm{x}$ & & $\mathrm{x}$ & \\
\hline Darwin & [155] & & $\mathrm{x}$ & $\mathrm{x}$ & & $\mathrm{x}$ & \\
\hline CrowdSense@Place & {$[147]$} & $\mathrm{x}$ & & & $\mathrm{x}$ & & $\mathrm{x}$ \\
\hline ILR & [168] & $\mathrm{x}$ & & & $\mathrm{x}$ & $\mathrm{x}$ & \\
\hline SoundSense & [166] & $\mathrm{x}$ & & $\mathrm{x}$ & & $\mathrm{x}$ & \\
\hline Urban WiFi & [164] & $\mathrm{x}$ & & & $\mathrm{x}$ & & $\mathrm{x}$ \\
\hline LiveCompare & [133] & & $\mathrm{x}$ & $\mathrm{x}$ & & $\mathrm{x}$ & \\
\hline MobiClique & [150] & & $\mathrm{x}$ & $\mathrm{x}$ & & $\mathrm{x}$ & \\
\hline MobiShop & [132] & & $\mathrm{x}$ & $\mathrm{x}$ & & $\mathrm{x}$ & \\
\hline SPA & [134] & $\mathrm{x}$ & & & $\mathrm{x}$ & & $\mathrm{x}$ \\
\hline EmotionSense & [149] & $\mathrm{x}$ & & & $\mathrm{x}$ & & $\mathrm{x}$ \\
\hline ConferenceSense & [167] & & $\mathrm{x}$ & $\mathrm{x}$ & & $\mathrm{x}$ & \\
\hline Travel Packages & [169] & & $\mathrm{x}$ & $\mathrm{x}$ & & $\mathrm{x}$ & \\
\hline Mahali & [129] & $\mathrm{x}$ & & & $\mathrm{x}$ & & $\mathrm{x}$ \\
\hline Ear-Phone & {$[122]$} & $\mathrm{x}$ & & $\mathrm{x}$ & & & $\mathrm{x}$ \\
\hline WreckWatch & [143] & & $\mathrm{x}$ & $\mathrm{x}$ & & $\mathrm{x}$ & \\
\hline VTrack & {$[145]$} & $\mathrm{x}$ & & & $\mathrm{x}$ & & $\mathrm{x}$ \\
\hline Social Serendipity & [151] & $\mathrm{x}$ & & $\mathrm{x}$ & & $\mathrm{x}$ & \\
\hline SociableSense & {$[152]$} & $\mathrm{x}$ & & & $\mathrm{x}$ & $\mathrm{x}$ & \\
\hline WhozThat & {$[153]$} & $\mathrm{x}$ & & $\mathrm{x}$ & & $\mathrm{x}$ & \\
\hline MoVi & {$[154]$} & & $\mathrm{x}$ & & $\mathrm{x}$ & $\mathrm{x}$ & \\
\hline
\end{tabular}

region of interest and for the total sensing time to accurately map the phenomena under analysis. Other applications requiring a continuous sensing consist in characterizing the coverage of WiFi intensity [164]. While mobile social networks usually represent event-based sensing, some exceptions presents a continuous contribution [149], [151]-[153]. Event-based MCS systems sense and report data when a certain event takes place, which can be related to context awareness, for instance when automatically detecting a car accident [143] or to a direct action from a user, such as recommending travels to others [169]. Typical examples are mobile social networks [59], [60], [150], [155] or health care applications [17], [19], [136], where users sense and share in particular moments. Other examples are comparing the prices of goods [132], [133], monitoring bus arrival time while waiting [142], and sending audio [167] or video [154] samples.

Responsibility. The responsibility of sensing and reporting is on mobile devices when users or the device itself with an application running on background decide when to sample according to the allocated task, independently to the central collector or other devices. The decision process typically depends on mobile devices in mobile social networks [59], [60], [150], [151], [153], [155], healthcare [17], [19], [136], e-commerce [132], [133], and environmental and road monitoring [20], [21], [115], [122], [123], [142], [143] The decision depends on the central collector when it is needed a general knowledge of the situation, for instance when more samples are needed from a certain region of interest. Characterizing WiFi [164], space weather [129], estimating the traffic delay [145] or improving the location reliability [168] require a central collector approach.

User involvement. It includes the participatory approach and the opportunistic one. MCS systems require a participatory approach when users exploit a sensor that needs to be activated or when human intelligence is required to detect a particular situation. A typical application that usually requires a participatory approach is health care, e.g., to take pictures for a diet [17], [19], [136]. In mobile social networks, it is also required to share actively updates [60], [62], [151], [153], [155]. Some works require users to report their impact about environmental [121] or emotional situations [149]. Comparing 
prices of goods requires users taking pictures and sharing them [133], [132]. Opportunistic approach is employed when devices are responsible for sensing. Noise [115], [122], [123] and air quality [21], [22], [56] monitoring map places with automatic sampling performed by mobile devices. Intelligent transportation systems also typically exploit an application running on the background without any user intervention, such as monitoring traffic and road conditions [20] [145], or bus arrival time [142]. Mapping WiFi intensity is another application that does not require active user participation [164].

\section{DISCUSSION}

The objective of this section is twofold. On the one hand, we provide a retrospective analysis of the past MCS research to expose the most prominent upcoming challenges and application scenarios. On the other hand, we present interdisciplinary connections between MCS and other research areas.

\section{A. Looking Back to See What's Next}

A decade has passed since the appearance of the first pillar works on MCS. In such a period, a number of successful and less successful proposals were developed, hence in the following paragraphs we summarize main insights and lessons learned. In these years, researchers have deeply investigated several aspects (e.g., user recruitment, task allocation, incentives mechanisms for participation, privacy) of MCS solutions in different application domains (e.g., healthcare, intelligent transportation systems, public safety). In the meanwhile, the MCS scenario has incredibly evolved because of a number of factors. First, new communications technologies will lay the foundation of next-generation MCS systems, such as 5G, Device-to-Device (D2D) communications, Mobile Edge Computing (MEC). Second, smart mobility and related services are modifying the citizens' behavior in moving and reaching places. Bike sharing, carpooling, new public transport modalities are rapidly and consistently modifying pedestrian mobility patterns and places reachability. This unleashes a higher level of pervasiveness for MCS systems. In addition, more sources to aggregate data to smart mobile devices should be taken into account (e.g., connected vehicles). All these considerations influence considerably MCS systems and the approach of organizers to design a crowdsensing campaign.

One of the most challenging issues MCS systems face is to deal with data potentially unreliable or malicious due to cheap sensors or misleading user behavior. Mobile devices measurements are simply imperfect because their standard sensors are mostly not designed for scientific applications, but considering size, cheap cost, limited power consumption, and functionality. To give some representative examples, accelerometers are typically affected by basic signal processing problems, such as noise, temporal jitter [118], [299], which consequently provide incomplete and unreliable data limiting the accuracy of several applications, e.g., activity recognition, road surface monitoring and everything related to mobile pattern recognition.

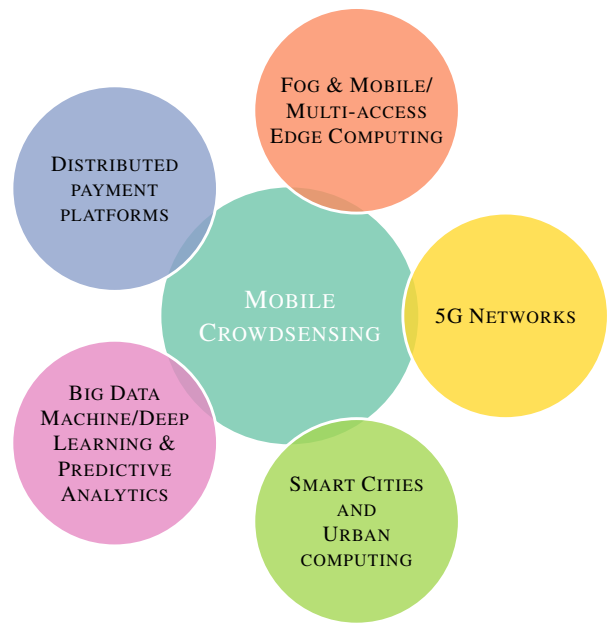

Fig. 15. Connections with other research areas

Despite the challenges, MCS has revealed a win-win strategy when used as a support and to improve existing monitoring infrastructure for its capacity to increase coverage and contextawareness. Citizens are seen as a connection to enhance the relationship between a city and its infrastructure. To give some examples, crowdsensed aggregated data is used in civil engineering for infrastructure management to observe the operational behavior of a bridge monitoring structural vibrations. The use case in the Harvard Bridge (Boston, US), has shown that data acquired from accelerometer of mobile devices in cars provide considerable information on the modal frequencies of the bridge [30]. Asfault is a system that monitors road conditions by performing ML and signal processing techniques on data collected from accelerometers embedded in mobile devices [300]. Detection of emergency situations through accelerometers, such as earthquakes [117], [119] are other fields of application. Creekwatch is an application that allows monitoring the conditions of the watershed through crowdsensed data, such as the flow rate [24]. Safestreet is an application that aggregates data from several smartphones to monitor the condition of the road surface for a safer driving and less risk of car accidents [144]. Glutensensor collects information about healthy food and shares information between celiac people, providing the possibility to map and rate restaurants and places [116]. CrowdMonitor is a crowdsensing approach in monitoring the emergency services through citizens movements and shared data, coordinating real and virtual activities and providing an overview of an emergency situation, overall in unreachable places [301].

\section{B. Inter-disciplinary Interconnections}

This section analyzes inter-disciplinary research areas where MCS plays an important role (e.g., smart cities and urban computing) or can benefit from novel technological advances (e.g., 5G networks and machine learning). Fig. 15 provides graphical illustration of the considered areas.

Smart cities and urban computing. Nowadays, half of the world population lives in cities and this percentage is projected 
to rise even more in the next years [302]. While nearly $2 \%$ of the world's surface is occupied by urban environments, cities contribute to $80 \%$ of global gas emission, $75 \%$ of global energy consumption [303], and $60 \%$ of residential water use [302]. For this reason, sustainable development usually with Information and Communication Technologies (ICT) plays a crucial role in city development [304]. While deploying new sensing infrastructures is typically expensive, MCS systems represent a win-win strategy. The interaction of human intelligence and mobility with sensing and communication capabilities of mobile devices provides higher accuracy and better context awareness compared to traditional sensor networks [305]. Urban computing has the precise objective of understanding and managing human activities in urban environments. This involves different aspects, such as urban morphology and corresponding street network (it has been proven that the configuration of the street network and the distribution of outdoor crimes are associated [162]), relation between citizens and point of interests (POIs), commuting required times, accessibility, and availability of transportations [306] (e.g., public transports, carpooling, bike sharing). Analyzing patterns of human flows and contacts, dynamics of residents and non-residents, and correlations with POIs or special events are few examples of scenarios where MCS can find applicability [307].

Big data, machine/deep learning, and predictive analytics. According to Cisco forecasts, the total amount of data created by Internet-connected devices will increase up to $847 \mathrm{ZB}$ per year by 2021 [308]. Such data originates from a wide range of fields and applications and exhibit high heterogeneity, large volume, variety, uncertain accuracy and dependency on application requirements. Big data analytics aims to inspect the contributed data and gain insights applicable to different purposes, such as monitoring phenomena, profiling user behaviors, unraveling information that leads to better conclusions than raw data [309] In the last years, big data analytics has revealed a successful emerging strategy in smart and connected communities, such as MCS systems, for real use cases [310].

Machine and deep learning techniques allow managing large and heterogeneous data volumes and applications in complex mobile architectures [311], such as MCS systems. Different ML techniques can be used to optimize the entire cycle of MCS paradigm, in particular, to maximize sensing quality while minimizing costs sustained by users [312]. Deep learning deals with large datasets by using back-propagation algorithms and allows computational models, that are composed of multiple processing layers, to learn representations of data with multiple levels of abstraction [313]. Crowdsensed data analyzed with neural networks techniques permits to predict human perceptual response to images [314].

Predictive analytics aims to predict future outcomes and events by exploiting statistical modeling and deep learning techniques. For example, foobot exploits learning techniques to detect patterns of air quality [315]. In intelligent transportation systems, deep learning techniques can be used to analyze spatio-temporal correlations to predict traffic flows and improve travel times [316], and to prevent pedestrian injuries and deaths [317]. In [318], the authors propose to take advantage of sensed data spatio-temporal correlations. By performing compressed-sensing and through deep Q-learning techniques, the system infers the values of sensing readings in uncovered areas. In [319], the authors indirectly rely on transfer learning to learns the parameters of the crowdsensing network from previously acquired data. The objective is to model and estimate the parameters of an unknown model. In [320], the authors propose to efficiently allocate tasks according to citizens' behavior and profile. They aim to profile users' preferences exploiting implicit feedback from their historical performance and to formalize the problem of users' reliability through a semi-supervised learning model.

Distributed payment platforms based on blockchains / smart contracts for data acquisition. Monetary rewarding, i.e., to distribute micro-payments according to specific criteria of the sensing campaign, is certainly one of the most powerful incentives for recruitment. To deliver micropayments among the participants requires distributed, trusted and reliable platforms that run smart contracts to move funds between the parties. A smart contract is a self-executing digital contract verified through peers, without the need for a central authority. In this context, blockchain technology provides the crowdsensing stakeholders with a transparent, unalterable, ordered ledger, enabling a decentralized and secure environment. It makes feasible to share services and resources, leading to the deployment of a marketplace of services between devices [321]. Hyperledger is an open-source project to develop and improve blockchain frameworks and platforms [322]. It is based on the idea that only collaborative software development can guarantee interoperability and transparency to adopt blockchain technologies in the commercial mainstream. Ethereum is a decentralized platform that allows developers to distribute payments on the basis of previously chosen instructions without a counterparty risk or the need of a middle authority [323]. These platforms enable developers to define the reward the citizens on the basis of several parameters that can be set on application-basis (e.g., amount of data, QoI, battery drain).

Fog computing and mobile edge computing/multi-access edge computing (MEC). The widespread diffusion of mobile and IoT devices challenges the cloud computing paradigm to fulfill the requirements for mobility support, context awareness, and low latency that are envisioned for 5G networks [324], [325]. Moving the intelligence closer to the mobile devices is a win-win strategy to quickly perform MCS operations such as recruitment in fog platforms [277], [326], [327]. A fog platform typically consists of many layers, some located in the proximity of end users. Each layer can consist of a large number of nodes with computation, communication, and storage capabilities, including routers, gateways, access points, and base stations [328]. While the concept of fog computing was introduced by Cisco and it is seen as an extension of cloud computing [329], MEC is standardized by ETSI to bring application-oriented capabilities in the core of the mobile operators' networks at a one-hop distance from the end-user [330]. To illustrate with few representative examples, RMCS is a Robust MCS framework that integrates edge computing based local processing and deep learning based data 
validation to reduce traffic load and transmission latency [331]. In [278], the authors propose a MEC architecture for MCS systems that decreases privacy threats and permits citizens to control the flow of contributed sensor data. EdgeSense is a crowdsensing framework based on edge computing architecture that works on top of a secured peer-to-peer network over the participants, aiming to extract environmental information in monitored areas [332].

5G networks. The objective of the fifth generation $(5 \mathrm{G})$ of cellular mobile networks is to support high mobility, massive connectivity, higher data rates, and lower latency. Unlike conventional mobile networks that were optimized for a specific objective (e.g., voice or data), all these requirements need to be supported simultaneously [333]. As discussed in Subsection VI-A1, significant architectural modifications to the $4 \mathrm{G}$ architecture are foreseen in 5G. While high data rates and massive connectivity requirements are extensions of services already supported in $4 \mathrm{G}$ systems such as enhanced mobile broadband (eMBB) and massive machine type communications (mMTC), ultra-reliable low-latency communications (URLLC) pose to $5 \mathrm{G}$ networks unprecedented challenges [334]. For the former two services, the use of mm-wave frequencies is of great help [335]. Instead, URLLC pose particular challenges to the mobile network and the 3GPP specification 38.211 (Release 15) supports several numerologies with a shorter transmission time interval (TTI). The shorter TTI duration, the shorter the buffering, and the processing times. However, scheduling all traffic with short TTI duration would adversely impact mobile broadband services like crowdsensing applications whose traffic can either be categorized as eMBB (e.g., video streams) or mMTC (sensor readings). Unlike URLLC, such types of traffic require a high data rate and benefit from conventional TTI duration.

\section{CONCluding Remarks}

As of the time of this writing, MCS is a well-established paradigm for urban sensing. In this paper, we provide a comprehensive survey of MCS systems, with the aim to consolidate its foundation and terminology that in literature appear to be often obscure of used with a variety of meanings. Specifically, we overview existing surveys in MCS and closelyrelated research areas by highlighting the novelty of our work. An important part of our work consisted in analyzing the time evolution of MCS during the last decade by including pillar works and the most important technological factors that have contributed to the rise of MCS. We proposed a four-layered architecture to characterize the works in MCS. The architecture allows to categorize all areas of the stack, from the application layer to the sensing and physical-layers passing through data and communication. Furthermore, the architecture allows to discriminate between domain-specific and general-purpose MCS data collection frameworks. We presented both theoretical works, such as those pertaining to the areas of operational research and optimization, and more practical ones, such as platforms, simulators, and datasets. We also provided a discussion on economics and data trading. We proposed a detailed taxonomy for each layer of the architecture, classifying important works of MCS systems accordingly and providing a further clarification on it. Finally, we provided a perspective of future research directions given the past efforts and discussed the inter-disciplinary interconnection of MCS with other research areas.

\section{REFERENCES}

[1] H. Falaki, D. Lymberopoulos, R. Mahajan, S. Kandula, and D. Estrin, "A first look at traffic on smartphones," in Proceedings of the 10th ACM SIGCOMM Conference on Internet Measurement, ser. IMC, 2010, pp. 281-287.

[2] H. T. Dinh, C. Lee, D. Niyato, and P. Wang, "A survey of mobile cloud computing: architecture, applications, and approaches," Wireless Communications and Mobile Computing, vol. 13, no. 18, pp. 1587-1611, 2013.

[3] "Gartner says global smartphone sales stalled in the fourth quarter of 2018," Gartner, 2019. [Online]. Available: https://www.gartner.com/en/newsroom/press-releases/2019-0221-gartner-says-global-smartphone-sales-stalled-in-the-fourth-quart

[4] "Gartner says worldwide wearable device sales to grow 26 percent in 2019," Gartner, 2018. [Online]. Available: https://www.gartner.com/en/newsroom/press-releases/2018-11 29-gartner-says-worldwide-wearable-device-sales-to-grow-

[5] "Wearable technology statistics and trends 2018," smartinsights, 2017. [Online]. Available: https://www.smartinsights.com/digital-marketingstrategy/wearables-statistics-2017/

[6] MarketsandMarkets, "Crowd analytics market worth \$1,142.5 million by 2021," 2018. [Online]. Available: https://www.marketsandmarkets. com/PressReleases/crowd-analytics.asp

[7] R. Ganti, F. Ye, and H. Lei, "Mobile crowdsensing: current state and future challenges," IEEE Communications Magazine, vol. 49, no. 11, pp. 32-39, Nov 2011.

[8] N. Lane, E. Miluzzo, H. Lu, D. Peebles, T. Choudhury, and A. Campbell, "A survey of mobile phone sensing," IEEE Communications Magazine, vol. 48, no. 9, pp. 140-150, Sep 2010.

[9] W. Khan, Y. Xiang, M. Aalsalem, and Q. Arshad, "Mobile phone sensing systems: A survey," IEEE Communications Surveys Tutorials, vol. 15, no. 1, pp. 402-427, First Quarter 2013.

[10] B. Guo, Z. Yu, X. Zhou, and D. Zhang, "From participatory sensing to mobile crowd sensing," in IEEE International Conference on Pervasive Computing and Communications Workshops (PERCOM Workshops), Mar 2014, pp. 593-598.

[11] J. Sun, "Incentive mechanisms for mobile crowd sensing: Current states and challenges of work," CoRR, vol. abs/1310.8364, 2013 [Online]. Available: http://arxiv.org/abs/1310.8364

[12] X. Zhang, Z. Yang, W. Sun, Y. Liu, S. Tang, K. Xing, and X. Mao, "Incentives for mobile crowd sensing: A survey," IEEE Communications Surveys Tutorials, vol. 18, no. 1, pp. 54-67, First Quarter 2016.

[13] L. Jaimes, I. Vergara-Laurens, and A. Raij, "A survey of incentive techniques for mobile crowd sensing," IEEE Internet of Things Journal, vol. 2, no. 5, pp. 370-380, Oct 2015.

[14] K. Ota, M. Dong, J. Gui, and A. Liu, "Quoin: Incentive mechanisms for crowd sensing networks," IEEE Network, vol. 32, no. 2, pp. 114-119, Mar 2018.

[15] L. Pournajaf, L. Xiong, D. A. Garcia-Ulloa, and V. Sunderam, "A survey on privacy in mobile crowd sensing task management," Technical Report TR-2014-002, Department of Mathematics and Computer Science, Emory University, 2014.

[16] D. Christin, A. Reinhardt, S. S. Kanhere, and M. Hollick, "A survey on privacy in mobile participatory sensing applications," Journal of Systems and Software, vol. 84, no. 11, pp. 1928-1946, 2011.

[17] C. Gao, F. Kong, and J. Tan, "Healthaware: Tackling obesity with health aware smart phone systems," in IEEE International Conference on Robotics and Biomimetics (ROBIO), 2009, pp. 1549-1554.

[18] G. Chen, B. Yan, M. Shin, D. Kotz, and E. Berke, "MPCS: Mobilephone based patient compliance system for chronic illness care," in IEEE Annual International Mobile and Ubiquitous Systems: Networking \& Services, 2009, pp. 1-7.

[19] S. Reddy, A. Parker, J. Hyman, J. Burke, D. Estrin, and M. Hansen, "Image browsing, processing, and clustering for participatory sensing: lessons from a DietSense prototype," in Proceedings of the ACM Workshop on Embedded Networked Sensors, 2007, pp. 13-17. 
[20] P. Mohan, V. N. Padmanabhan, and R. Ramjee, "Nericell: rich monitoring of road and traffic conditions using mobile smartphones," in Proceedings of the ACM Conference on Embedded Network Sensor Systems, 2008, pp. 323-336.

[21] D. Hasenfratz, O. Saukh, S. Sturzenegger, and L. Thiele, "Participatory air pollution monitoring using smartphones," in Mobile Sensing: From Smartphones and Wearables to Big Data. ACM, Apr 2012.

[22] V. Sivaraman, J. Carrapetta, K. Hu, and B. G. Luxan, "HazeWatch: A participatory sensor system for monitoring air pollution in Sydney," in IEEE Conference on Local Computer Networks (LCN) - Workshops, Oct 2013, pp. 56-64.

[23] L. Liu, W. Liu, Y. Zheng, H. Ma, and C. Zhang, "Third-Eye: A mobilephone-enabled crowdsensing system for air quality monitoring," Proceedings of the ACM on Interactive, Mobile, Wearable and Ubiquitous Technologies, vol. 2, no. 1, pp. 1-26, Mar. 2018.

[24] S. Kim, C. Robson, T. Zimmerman, J. Pierce, and E. M. Haber, "Creek Watch: Pairing usefulness and usability for successful citizen science," in Proc. of the ACM Conference on Human Factors in Computing Systems, ser. CHI, 2011, pp. 2125-2134.

[25] S. Reddy and V. Samanta, "Urban Sensing: Garbage Watch," 2011, UCLA Center for Embedded Networked Sensing.

[26] D. Bonino, M. T. D. Alizo, C. Pastrone, and M. Spirito, "WasteApp Smarter waste recycling for smart citizens," in International Multidisci plinary Conference on Computer and Energy Science (SpliTech), Jul 2016, pp. 1-6.

[27] O. Alvear, C. T. Calafate, J.-C. Cano, and P. Manzoni, "Crowdsensing in smart cities: Overview, platforms, and environment sensing issues," Sensors, vol. 18, no. 2, 2018

[28] H. Schaffers, N. Komninos, M. Pallot, B. Trousse, M. Nilsson, and A. Oliveira, "Smart cities and the future Internet: Towards cooperation frameworks for open innovation," in The Future Internet, ser. Lecture Notes in Computer Science. Springer Berlin Heidelberg, 2011, vol. 6656, pp. 431-446.

[29] A. Zanella, N. Bui, A. Castellani, L. Vangelista, and M. Zorzi, "Internet of Things for smart cities," IEEE Internet of Things Journal, vol. 1 , no. 1, pp. 22-32, Feb 2014

[30] T. J. Matarazzo, P. Santi, S. N. Pakzad, K. Carter, C. Ratti, B. Moaveni, C. Osgood, and N. Jacob, "Crowdsensing framework for monitoring bridge vibrations using moving smartphones," Proceedings of the IEEE, vol. 106, no. 4, pp. 577-593, Apr 2018.

[31] K. Farkas, G. Feher, A. Benczur, and C. Sidlo, "Crowdsending based public transport information service in smart cities," IEEE Communications Magazine, vol. 53, no. 8, pp. 158-165, Aug 2015.

[32] M. Moreno, A. Skarmeta, and A. Jara, "How to intelligently make sense of real data of smart cities," in International Conference on Recent Advances in Internet of Things (RIoT), Apr 2015, pp. 1-6.

[33] S. Nawaz, C. Efstratiou, and C. Mascolo, "ParkSense: A smartphone based sensing system for on-street parking," in Proceedings of the ACM Conference on Mobile Computing and Networking, ser. MobiCom, 2013 pp. $75-86$.

[34] J. Cherian, J. Luo, H. Guo, S. S. Ho, and R. Wisbrun, "ParkGauge: Gauging the occupancy of parking garages with crowdsensed parking characteristics," in IEEE International Conference on Mobile Data Management (MDM), vol. 1, Jun 2016, pp. 92-101.

[35] B. Guo, Z. Wang, Z. Yu, Y. Wang, N. Y. Yen, R. Huang, and X. Zhou, "Mobile crowd sensing and computing: The review of an emerging human-powered sensing paradigm," ACM Comput. Surv., vol. 48, no. 1, pp. 1-31, Aug. 2015.

[36] Y. Han, Y. Zhu, and J. Yu, "A distributed utility-maximizing algorithm for data collection in mobile crowd sensing," in IEEE Global Communications Conference (GLOBECOM), Dec 2014, pp. 277-282.

[37] H. Ma, D. Zhao, and P. Yuan, "Opportunities in mobile crowd sensing," IEEE Communications Magazine, vol. 52, no. 8, pp. 29-35, Aug 2014.

[38] P. Jayaraman, C. Perera, D. Georgakopoulos, and A. Zaslavsky, "Efficient opportunistic sensing using mobile collaborative platform MOSDEN," in International Conference Conference on Collaborative Computing: Networking, Applications and Worksharing (Collaboratecom), Oct 2013, pp. 77-86.

[39] W. Gong, B. Zhang, and C. Li, "Task assignment in mobile crowdsensing: Present and future directions," IEEE Network, pp. 1-8, 2018.

[40] B. Guo, Q. Han, H. Chen, L. Shangguan, Z. Zhou, and Z. Yu, "The emergence of visual crowdsensing: Challenges and opportunities," IEEE Communications Surveys Tutorials, vol. 19, no. 4, pp. 2526-2543, Fourth Quarter 2017.

[41] Z. Xu, L. Mei, K.-K. R. Choo, Z. Lv, C. Hu, X. Luo, and Y. Liu, "Mobile crowd sensing of human-like intelligence using social sensors: A survey," Neurocomputing, pp. -, 2017.
[42] J. Phuttharak and S. W. Loke, "A review of mobile crowdsourcing architectures and challenges: Towards crowd-empowered Internet-ofThings," IEEE Access, pp. 1-22, Dec 2018.

[43] J. Liu, H. Shen, H. S. Narman, W. Chung, and Z. Lin, "A survey of mobile crowdsensing techniques: A critical component for the Internet of Things," ACM Transactions on Cyber-Physical Systems, vol. 2, no. 3, pp. 1-26, Jun 2018.

[44] K. Abualsaud, T. M. Elfouly, T. Khattab, E. Yaacoub, L. S. Ismail, M. H. Ahmed, and M. Guizani, "A survey on mobile crowd-sensing and its applications in the IoT era," IEEE Access, vol. 7, pp. 3855-3881, 2019.

[45] I. F. Akyildiz, W. Su, Y. Sankarasubramaniam, and E. Cayirci, "A survey on sensor networks," IEEE Communications Magazine, vol. 40, no. 8, pp. 102-114, Aug. 2002.

[46] J. Yick, B. Mukherjee, and D. Ghosal, "Wireless sensor network survey," Computer Networks, vol. 52, no. 12, pp. 2292 - 2330, 2008.

[47] P. Rawat, K. D. Singh, H. Chaouchi, and J. M. Bonnin, "Wireless sensor networks: a survey on recent developments and potential synergies," The Journal of Supercomputing, vol. 68, no. 1, pp. 1-48, Apr 2014.

[48] M. Liu, "A study of mobile sensing using smartphones," International Journal of Distributed Sensor Networks, vol. 9, no. 3, p. 272916, 2013.

[49] C. Toth and G. Jóźków, "Remote sensing platforms and sensors: A survey," ISPRS Journal of Photogrammetry and Remote Sensing, vol. 115, no. Supplement C, pp. 22 - 36, 2016.

[50] V. Pejovic and M. Musolesi, "Anticipatory mobile computing: A survey of the state of the art and research challenges," ACM Comput. Surv., vol. 47, no. 3, pp. 1-29, Apr. 2015.

[51] N. Bui, M. Cesana, S. A. Hosseini, Q. Liao, I. Malanchini, and J. Widmer, "A survey of anticipatory mobile networking: Context-based classification, prediction methodologies, and optimization techniques," IEEE Communications Surveys Tutorials, vol. 19, no. 3, pp. 1790-1821, Third Quarter 2017

[52] H. Gao, C. H. Liu, W. Wang, J. Zhao, Z. Song, X. Su, J. Crowcroft, and K. K. Leung, "A survey of incentive mechanisms for participatory sensing," IEEE Communications Surveys Tutorials, vol. 17, no. 2, pp. 918-943, Second Quarter 2015.

[53] F. Restuccia, S. K. Das, and J. Payton, "Incentive mechanisms for participatory sensing: Survey and research challenges," ACM Transactions on Sensor Networks (TOSN), vol. 12, no. 2, pp. 1-40, Apr 2016.

[54] F. Restuccia, N. Ghosh, S. Bhattacharjee, S. K. Das, and T. Melodia, "Quality of information in mobile crowdsensing: Survey and research challenges," ACM Transactions on Sensor Networks (TOSN), vol. 13, no. 4, pp. 1-43, Nov 2017.

[55] A. Overeem, J. R Robinson, H. Leijnse, G.-J. Steeneveld, B. P Horn, and R. Uijlenhoet, "Crowdsourcing urban air temperatures from smartphone battery temperatures," Geophysical Research Letters, vol. 40, no. 15, pp. 4081-4085, 2013.

[56] P. Dutta, P. M. Aoki, N. Kumar, A. Mainwaring, C. Myers, W. Willett, and A. Woodruff, "Common sense: participatory urban sensing using a network of handheld air quality monitors," in Proc. of the ACM Conference on Embedded Networked Sensor Systems, 2009, pp. 349350.

[57] J. Howe, "The rise of crowdsourcing," Wired, Jan 2006. [Online] Available: https://www.wired.com/2006/06/crowds/

[58] J. A. Burke, D. Estrin, M. Hansen, A. Parker, N. Ramanathan, S. Reddy, and M. B. Srivastava, "Participatory sensing," Center for Embedded Network Sensing, 2006.

[59] E. Miluzzo, N. D. Lane, K. Fodor, R. Peterson, H. Lu, M. Musolesi, S. B Eisenman, X. Zheng, and A. T. Campbell, "Sensing meets mobile social networks: the design, implementation and evaluation of the cenceme application," in Proceedings of the ACM Conference on Embedded Network Sensor Systems, 2008, pp. 337-350.

[60] S. Gaonkar, J. Li, R. R. Choudhury, L. Cox, and A. Schmidt, "Microblog: sharing and querying content through mobile phones and social participation," in Proc. of the ACM International Conference on Mobile Systems, Applications, and Services, 2008, pp. 174-186.

[61] G. Cardone, L. Foschini, P. Bellavista, A. Corradi, C. Borcea, M. Talasila, and R. Curtmola, "Fostering participaction in smart cities: a geo-social crowdsensing platform," IEEE Communications Magazine, vol. 51, no. 6 , pp. 112-119, Jun 2013.

[62] N. Vastardis and K. Yang, "Mobile social networks: Architectures, social properties, and key research challenges," IEEE Communication Surveys \& Tutorials, vol. 15, no. 3, pp. 1355-1371, 2013.

[63] P. P. Jayaraman, C. Perera, D. Georgakopoulos, and A. Zaslavsky, "Efficient opportunistic sensing using mobile collaborative platform mosden," in International Conference Conference on Collaborative Computing: 
Networking, Applications and Worksharing (Collaboratecom), 2013, pp $77-86$.

[64] G. Cardone, A. Cirri, A. Corradi, and L. Foschini, "The ParticipAct mobile crowd sensing living lab: The testbed for smart cities," IEEE Communications Magazine, vol. 52, no. 10, pp. 78-85, Oct 2014.

[65] B. Kantarci and H. T. Mouftah, "Trustworthy sensing for public safety in cloud-centric Internet of Things," IEEE Internet of Things Journal, vol. 1, no. 4, pp. 360-368, Aug 2014.

[66] C. Tanas and J. Herrera-Joancomartí, "Crowdsensing simulation using ns-3," Citizen in Sensor Networks: Second International Workshop, CitiSens, pp. 47-58, 2014, Springer International Publishing.

[67] S. Chessa, A. Corradi, L. Foschini, and M. Girolami, "Empowering mobile crowdsensing through social and ad hoc networking," IEEE Communications Magazine, vol. 54, no. 7, pp. 108-114, Jul 2016.

[68] C. Fiandrino, A. Capponi, G. Cacciatore, D. Kliazovich, U. Sorger, P. Bouvry, B. Kantarci, F. Granelli, and S. Giordano, "CrowdSenSim a simulation platform for mobile crowdsensing in realistic urban environments," IEEE Access, vol. 5, pp. 3490-3503, Feb 2017.

[69] "Runtastic." [Online]. Available: https://www.runtastic.com

[70] S. Consolvo, D. W. McDonald, T. Toscos, M. Y. Chen, J. Froehlich, B. Harrison, P. Klasnja, A. LaMarca, L. LeGrand, R. Libby et al., "Activity sensing in the wild: a field trial of UbiFit garden," in Proc. of the ACM Conference on Human Factors in Computing Systems, ser. SIGCHI, 2008, pp. 1797-1806.

[71] J. Dai, X. Bai, Z. Yang, Z. Shen, and D. Xuan, "PerFallD: A pervasive fall detection system using mobile phones," in IEEE International Conference on Pervasive Computing and Communications Workshops (PERCOM Workshops), 2010, pp. 292-297.

[72] Y. He, Y. Li, and S.-O. Bao, "Fall detection by built-in tri-accelerometer of smartphone," in IEEE EMBS International Conference on Biomedical and Health Informatics (BHI), 2012, pp. 184-187.

[73] J. R. Kwapisz, G. M. Weiss, and S. A. Moore, "Activity recognition using cell phone accelerometers," ACM SigKDD Explorations Newsletter, vol. 12 , no. 2, pp. 74-82, 2011.

[74] M. A. Habib, M. S. Mohktar, S. B. Kamaruzzaman, K. S. Lim, T. M Pin, and F. Ibrahim, "Smartphone-based solutions for fall detection and prevention: challenges and open issues," Sensors, vol. 14, no. 4, pp. 7181-7208, 2014.

[75] S. Wang, C. Chen, and J. Ma, "Accelerometer based transportation mode recognition on mobile phones," in Asia-Pacific Conference on Wearable Computing Systems (APWCS). IEEE, 2010, pp. 44-46.

[76] S. Reddy, J. Burke, D. Estrin, M. Hansen, and M. Srivastava, "Determining transportation mode on mobile phones," in IEEE International Symposium on Wearable Computers (ISWC), 2008, pp. 25-28.

[77] S. Hemminki, P. Nurmi, and S. Tarkoma, "Accelerometer-based transportation mode detection on smartphones," in Proc. of the ACM Conference on Embedded Networked Sensor Systems, ser. SenSys, 2013 p. 13.

[78] S. Reddy, M. Mun, J. Burke, D. Estrin, M. Hansen, and M. Srivastava, "Using mobile phones to determine transportation modes," $A C M$ Transactions on Sensor Networks (TOSN), vol. 6, no. 2, p. 13, 2010.

[79] Y. Michalevsky, D. Boneh, and G. Nakibly, "Gyrophone: Recognizing speech from gyroscope signals," in Proc. 23rd USENIX Security Symposium, 2014.

[80] D. Johnson, M. M. Trivedi et al., "Driving style recognition using a smartphone as a sensor platform," in IEEE International Conference on Intelligent Transportation Systems (ITSC), 2011, pp. 1609-1615.

[81] H. Ye, T. Gu, X. Tao, and J. Lu, "B-Loc: scalable floor localization using barometer on smartphone," in IEEE International Conference on Mobile Ad Hoc and Sensor Systems (MASS), 2014, pp. 127-135.

[82] S. Vanini and S. Giordano, "Adaptive context-agnostic floor transition detection on smart mobile devices," in IEEE International Conference on Pervasive Computing and Communications Workshops (PERCOM Workshops), 2013, pp. 2-7.

[83] K. Komeda, M. Mochizuki, and N. Nishiko, "User activity recognition method based on atmospheric pressure sensing," in Proceedings of the ACM International Conference on Pervasive and Ubiquitous Computing, 2014, pp. 737-746.

[84] P. Castillejo, J.-F. Martínez, L. López, and G. Rubio, "An Internet of Things approach for managing smart services provided by wearable devices," International Journal of Distributed Sensor Networks, vol. 9 , no. 2, p. 190813, 2013.

[85] X. Lai, Q. Liu, X. Wei, W. Wang, G. Zhou, and G. Han, "A survey of body sensor networks," Sensors, vol. 13, no. 5, pp. 5406-5447, 2013.

[86] M. Ghamari, B. Janko, R. S. Sherratt, W. Harwin, R. Piechockic, and C. Soltanpur, "A survey on wireless body area networks for eHealthcare systems in residential environments," Sensors, vol. 16, no. 6, 2016.
[87] A. Filippeschi, N. Schmitz, M. Miezal, G. Bleser, E. Ruffaldi, and D. Stricker, "Survey of motion tracking methods based on inertial sensors: A focus on upper limb human motion," Sensors, vol. 17, no. 6, 2017.

[88] E. Estellés-Arolas and F. González-Ladrón-De-Guevara, "Towards an integrated crowdsourcing definition," Journal of Information science, vol. 38, no. 2, pp. 189-200, Apr 2012.

[89] D. C. Brabham, "Crowdsourcing as a model for problem solving: An introduction and cases," pp. 75-90, 2008.

[90] J. M. Leimeister, M. Huber, U. Bretschneider, and H. Krcmar, "Leveraging crowdsourcing: Activation-supporting components for IT-based ideas competition," Journal of Management Information Systems, vol. 26, no. 1, pp. 197-224, 2009.

[91] A. Kittur, E. H. Chi, and B. Suh, "Crowdsourcing user studies with Mechanical Turk," in Proceedings of the ACM Conference on Human Factors in Computing Systems, ser. SIGCHI, 2008, pp. 453-456.

[92] J. Ross, L. Irani, M. S. Silberman, A. Zaldivar, and B. Tomlinson, "Who are the crowdworkers?: Shifting demographics in mechanical turk," in Proceedings of the ACM Conference on Human Factors in Computing Systems, ser. SIGCHI EA, 2010, pp. 2863-2872.

[93] L. Duan, L. Huang, C. Langbort, A. Pozdnukhov, J. Walrand, and L. Zhang, "Human-in-the-Loop mobile networks: A survey of recent advancements," IEEE Journal on Selected Areas in Communications, vol. 35, no. 4, pp. 813-831, Apr 2017.

[94] E. Kamar, S. Hacker, and E. Horvitz, "Combining human and machine intelligence in large-scale crowdsourcing," in International Conference on Autonomous Agents and Multiagent Systems, ser. AAMAS, vol. 1. International Foundation for Autonomous Agents and Multiagent Systems, 2012, pp. 467-474.

[95] R. Fakoor, M. Raj, A. Nazi, M. Di Francesco, and S. K. Das, "An integrated cloud-based framework for mobile phone sensing," in Proceedings of the ACM Workshop on Mobile Cloud Computing, 2012, pp. 47-52.

[96] D. Huang, T. Xing, and H. Wu, "Mobile cloud computing service models: a user-centric approach," IEEE Network, vol. 27, no. 5, pp. 6-11, Sep 2013.

[97] B. Kantarci and H. T. Mouftah, "Sensing services in cloud-centric Internet of Things: A survey, taxonomy and challenges," in IEEE International Conference on Communication Workshop (ICCW), Jun 2015, pp. 1865-1870.

[98] X. Sheng, J. Tang, X. Xiao, and G. Xue, "Sensing as a Service: Challenges, solutions and future directions," IEEE Sensors Journal, vol. 13, no. 10, pp. 3733-3741, 2013.

[99] R. D. Lauro, F. Lucarelli, and R. Montella, "SIaaS - sensing instrument as a service using cloud computing to turn physical instrument into ubiquitous service," in IEEE 10th International Symposium on Parallel and Distributed Processing with Applications, Jul 2012, pp. 861-862.

[100] C. Doukas and I. Maglogiannis, "Bringing IoT and cloud computing towards pervasive Healthcare," in International Conference on Innovative Mobile and Internet Services in Ubiquitous Computing, Jul 2012, pp. $922-926$

[101] M. Boukhechba, A. R. Daros, K. Fua, P. I. Chow, B. A. Teachman, and L. E. Barnes, "DemonicSalmon: Monitoring mental health and social interactions of college students using smartphones," in IEEE/ACM Conference on Connected Health: Applications, Systems and Engineering Technologies (CHASE), Sep 2018.

[102] I. Khan, F. Belqasmi, R. Glitho, N. Crespi, M. Morrow, and P. Polakos, "Wireless sensor network virtualization: A survey," IEEE Communications Surveys Tutorials, vol. 18, no. 1, pp. 553-576, First Quarter 2016.

[103] H. I. Kobo, A. M. Abu-Mahfouz, and G. P. Hancke, "A survey on software-defined wireless sensor networks: Challenges and design requirements," IEEE Access, vol. 5, pp. 1872-1899, 2017.

[104] I. Ali, A. Gani, I. Ahmedy, I. Yaqoob, S. Khan, and M. H. Anisi, "Data collection in smart communities using sensor cloud: Recent advances, taxonomy, and future research directions," IEEE Communications Magazine, vol. 56, no. 7, pp. 192-197, 2018.

[105] A. B. Zaslavsky, C. Perera, and D. Georgakopoulos, "Sensing as a Service and Big Data," CoRR, vol. abs/1301.0159, 2013. [Online]. Available: http://arxiv.org/abs/1301.0159

[106] S. Alam, M. M. R. Chowdhury, and J. Noll, "SenaaS: An eventdriven sensor virtualization approach for Internet of Things cloud," in IEEE International Conference on Networked Embedded Systems for Enterprise Applications, Nov 2010, pp. 1-6.

[107] S. Distefano, G. Merlino, and A. Puliafito, "Sensing and actuation as a service: A new development for clouds," in IEEE International 
Symposium on Network Computing and Applications, Aug 2012, pp 272-275.

[108] G. Merlino, S. Arkoulis, S. Distefano, C. Papagianni, A. Puliafito, and S. Papavassiliou, "Mobile crowdsensing as a service: A platform for applications on top of sensing clouds," Future Generation Computer Systems, vol. 56, pp. $623-639,2016$.

[109] "MaaS as a concept," MaaS Finland. [Online]. Available: http: //maas.global/maas-as-a-concept/

[110] S. A. Rahman, A. Mourad, M. E. Barachi, and W. A. Orabi, "A novel ondemand vehicular sensing framework for traffic condition monitoring," Vehicular Communications, vol. 12, pp. 165 - 178, 2018.

[111] A. Capponi, C. Fiandrino, C. Franck, U. Sorger, D. Kliazovich, and P. Bouvry, "Assessing performance of Internet of Things-based mobile crowdsensing systems for sensing as a service applications in smart cities," in IEEE International Conference on Cloud Computing Technology and Science (CloudCom), Dec 2016, pp. 456-459.

[112] K. Han, C. Zhang, and J. Luo, "Taming the uncertainty: Budget limited robust crowdsensing through online learning," IEEE/ACM Transactions on Networking, vol. 24, no. 3, pp. 1462-1475, Jun 2016.

[113] S. Satpathy, B. Sahoo, and A. K. Turuk, "Sensing and actuation as a service delivery model in cloud edge centric Internet of Things," Future Generation Computer Systems, vol. 86, pp. 281 - 296, 2018.

[114] R. Fakoor, M. Raj, A. Nazi, M. Di Francesco, and S. K. Das, "An integrated cloud-based framework for mobile phone sensing," in Proc. of the ACM Workshop on Mobile Cloud Computing, ser. MCC, 2012, pp. $47-52$.

[115] I. Schweizer, R. Bärtl, A. Schulz, F. Probst, and M. Mühläuser, "Noisemap - real-time participatory noise maps," in International Workshop on Sensing Applications on Mobile Phones, 2011, pp. 15.

[116] 6sensorlabs. (2015) Gluten sensor. [Online].

[117] J. Reilly, S. Dashti, M. Ervasti, J. D. Bray, S. D. Glaser, and A. M. Bayen, "Mobile phones as seismologic sensors: Automating data extraction for the ishake system," IEEE Transactions on Automation Science and Engineering, vol. 10, no. 2, pp. 242-251, Apr 2013.

[118] S. Dashti, J. D. Bray, J. Reilly, S. Glaser, A. Bayen, and E. Mari, "Evaluating the reliability of phones as seismic monitoring instruments," Earthquake Spectra, vol. 30, no. 2, pp. 721-742, 2014.

[119] M. Faulkner, R. Clayton, T. Heaton, K. M. Chandy, M. Kohler, J. Bunn, R. Guy, A. Liu, M. Olson, M. Cheng, and A. Krause, "Community sense and response systems: Your phone as quake detector," ACM Commun. vol. 57, no. 7, pp. 66-75, Jul 2014.

[120] Y. Ishigaki, Y. Matsumoto, R. Ichimiya, and K. Tanaka, "Development of mobile radiation monitoring system utilizing smartphone and its field tests in fukushima," IEEE Sensors Journal, vol. 13, no. 10, pp. 3520-3526, 2013

[121] M. Mun, S. Reddy, K. Shilton, N. Yau, J. Burke, D. Estrin, M. Hansen, E. Howard, R. West, and P. Boda, "PEIR, the personal environmental impact report, as a platform for participatory sensing systems research," in Proc. of the ACM International Conference on Mobile Systems, Applications, and Services, 2009, pp. 55-68.

[122] R. K. Rana, C. T. Chou, S. S. Kanhere, N. Bulusu, and W. Hu, "Earphone: an end-to-end participatory urban noise mapping system," in Proc of the ACM/IEEE International Conference on Information Processing in Sensor Networks, 2010, pp. 105-116.

[123] N. Maisonneuve, M. Stevens, M. E. Niessen, and L. Steels, "NoiseTube: Measuring and mapping noise pollution with mobile phones," in Information Technologies in Environmental Engineering. Springer, 2009, pp. 215-228.

[124] M. Stevens and E. D'Hondt, "Crowdsourcing of pollution data using smartphones," in Workshop on Ubiquitous Crowdsourcing, 2010.

[125] F. Montori, L. Bedogni, and L. Bononi, "A collaborative Internet of Things architecture for smart cities and environmental monitoring," IEEE Internet of Things Journal, vol. 5, no. 2, pp. 592-605, Apr 2018

[126] C. Xiang, P. Yang, and S. Xiao, "Counter-strike: accurate and robust identification of low-level radiation sources with crowd-sensing networks," Personal and Ubiquitous Computing, vol. 21, no. 1, pp. 75-84, Feb 2017.

[127] M. Budde, P. Barbera, R. El Masri, T. Riedel, and M. Beigl, "Retrofitting smartphones to be used as particulate matter dosimeters," in Proc. of the ACM International Symposium on Wearable Computers, ser. ISWC 2013, pp. 139-140.

[128] D. A. Galvan, A. Komjathy, M. P. Hickey, P. Stephens, J. Snively, Y. Tony Song, M. D. Butala, and A. J. Mannucci, "Ionospheric signatures of tohoku-oki tsunami of march 11, 2011: Model comparisons near the epicenter," Radio Science, vol. 47, no. 4, 2012.
[129] V. Pankratius, F. Lind, A. Coster, P. Erickson, and J. Semeter, "Mobile crowd sensing in space weather monitoring: the Mahali project," IEEE Communications Magazine, vol. 52, no. 8, pp. 22-28, 2014.

[130] N. Bulusu, C. T. Chou, S. Kanhere, Y. Dong, S. Sehgal, D. Sullivan, and L. Blazeski, "Participatory sensing in commerce: Using mobile camera phones to track market price dispersion," in Proc. of the International Workshop on Urban, Community, and Social Applications of Networked Sensing Systems (UrbanSense), 2008, pp. 6-10.

[131] Y. F. Dong, S. Kanhere, C. T. Chou, and N. Bulusu, "Automatic collection of fuel prices from a network of mobile cameras," in Distributed computing in sensor systems. Springer, 2008, pp. 140-156.

[132] S. Sehgal, S. S. Kanhere, and C. T. Chou, "Mobishop: Using mobile phones for sharing consumer pricing information," in Demo Session of the Intl. Conference on Distributed Computing in Sensor Systems, 2008.

[133] L. Deng and L. P. Cox, "Livecompare: grocery bargain hunting through participatory sensing," in Proc. of the ACM Workshop on Mobile Computing Systems and Applications, 2009, p. 4.

[134] K. Sha, G. Zhan, W. Shi, M. Lumley, C. Wiholm, and B. Arnetz, "SPA: a smart phone assisted chronic illness self-management system with participatory sensing," in Proceedings of the ACM Workshop on Systems and Networking Support for Health Care and Assisted Living Environments, 2008, p. 5.

[135] W.-J. Yi, W. Jia, and J. Saniie, "Mobile sensor data collector using Android smartphone," in IEEE 55th International Midwest Symposium on Circuits and Systems (MWSCAS), 2012, pp. 956-959.

[136] J. Hicks, N. Ramanathan, D. Kim, M. Monibi, J. Selsky, M. Hansen, and D. Estrin, "AndWellness: an open mobile system for activity and experience sampling," in Wireless Health. ACM, 2010, pp. 34-43.

[137] Q. Xu and R. Zheng, "MobiBee: A mobile treasure hunt game for location-dependent fingerprint collection," in Proceedings of the ACM International Joint Conference on Pervasive and Ubiquitous Computing: Adjunct, 2016, pp. 1472-1477.

[138] B. Zhou, Q. Li, Q. Mao, and W. Tu, "A robust crowdsourcing-based indoor localization system," Sensors, vol. 17, no. 4, p. 864, 2017.

[139] Q. Xu, R. Zheng, and E. Tahoun, "Detecting location fraud in indoor mobile crowdsensing," in Proceedings of the First ACM Workshop on Mobile Crowdsensing Systems and Applications, 2017, pp. 44-49.

[140] F. Calabrese, M. Colonna, P. Lovisolo, D. Parata, and C. Ratti, "Realtime urban monitoring using cell phones: A case study in Rome," IEEE Transactions on Intelligent Transportation Systems, vol. 12, no. 1, pp. 141-151, Mar 2011.

[141] R. Tse, Y. Xiao, G. Pau, M. Roccetti, S. Fdida, and G. Marfia, "On the feasibility of social network-based pollution sensing in ITSs," arXiv preprint arXiv:1411.6573, 2014

[142] P. Zhou, Y. Zheng, and M. Li, "How long to wait? predicting bus arrival time with mobile phone based participatory sensing," in Proc. of the ACM International Conference on Mobile Systems, Applications, and Services, 2012, pp. 379-392.

[143] J. White, C. Thompson, H. Turner, B. Dougherty, and D. C. Schmidt, "Wreckwatch: Automatic traffic accident detection and notification with smartphones," Mobile Networks and Applications, vol. 16, no. 3, pp. 285-303, 2011.

[144] V. Singh, D. Chander, U. Chhaparia, and B. Raman, "SafeStreet: An automated road anomaly detection and early-warning system using mobile crowdsensing," in IEEE International Conference on Communication Systems Networks (COMSNETS), Jan 2018, pp. 549552.

[145] A. Thiagarajan, L. Ravindranath, K. LaCurts, S. Madden, H. Balakrishnan, S. Toledo, and J. Eriksson, "VTrack: accurate, energy-aware road traffic delay estimation using mobile phones," in Proceedings of the ACM Conference on Embedded Networked Sensor Systems, ser. SenSys, 2009, pp. 85-98.

[146] M. A. Rahman and M. S. Hossain, "A location-based mobile crowdsensing framework supporting a massive Ad Hoc social network environment," IEEE Communications Magazine, vol. 55, no. 3, pp. 76-85, Mar 2017.

[147] Y. Chon, N. D. Lane, Y. Kim, F. Zhao, and H. Cha, "Understanding the coverage and scalability of place-centric crowdsensing," in Proc. of the ACM international joint Conference on Pervasive and Ubiquitous Computing, ser. UbiComp, 2013, pp. 3-12.

[148] Y. Chon, N. D. Lane, F. Li, H. Cha, and F. Zhao, "Automatically characterizing places with opportunistic crowdsensing using smartphones," in Proceedings of the ACM Conference on Ubiquitous Computing, 2012, pp. 481-490.

[149] K. K. Rachuri, M. Musolesi, C. Mascolo, P. J. Rentfrow, C. Longworth, and A. Aucinas, "EmotionSense: a mobile phones based adaptive 
platform for experimental social psychology research," in Proceedings of the ACM International Conference on Ubiquitous Computing, 2010, pp. 281-290.

[150] A.-K. Pietiläinen, E. Oliver, J. LeBrun, G. Varghese, and C. Diot, "MobiClique: middleware for mobile social networking," in Proc. of the ACM Workshop on Online Social Networks, 2009, pp. 49-54.

[151] N. Eagle and A. Pentland, "Social serendipity: Mobilizing social software," IEEE Pervasive Computing, vol. 4, no. 2, pp. 28-34, 2005.

[152] K. K. Rachuri, C. Mascolo, M. Musolesi, and P. J. Rentfrow, "Sociablesense: exploring the trade-offs of adaptive sampling and computation offloading for social sensing," in Proceedings of the ACM International Conference on Mobile Computing and Networking, 2011, pp. 73-84.

[153] A. Beach, M. Gartrell, S. Akkala, J. Elston, J. Kelley, K. Nishimoto, B. Ray, S. Razgulin, K. Sundaresan, B. Surendar et al., "Whozthat? evolving an ecosystem for context-aware mobile social networks," IEEE Network, vol. 22, no. 4, pp. 50-55, 2008

[154] X. Bao and R. Roy Choudhury, "MoVi: mobile phone based video highlights via collaborative sensing," in Proceedings of the ACM International Conference on Mobile Systems, Applications, and Services, 2010, pp. 357-370.

[155] E. Miluzzo, C. T. Cornelius, A. Ramaswamy, T. Choudhury, Z. Liu, and A. T. Campbell, "Darwin phones: the evolution of sensing and inference on mobile phones," in Proc. of the ACM International Conference on Mobile Systems, Applications, and Services, 2010, pp. 5-20.

[156] J. Ballesteros, B. Carbunar, M. Rahman, N. Rishe, and S. Iyengar, "Towards safe cities: A mobile and social networking approach," IEEE Transactions on Parallel and Distributed Systems, vol. 25, no. 9, pp. 2451-2462, 2014.

[157] P. Fraga-Lamas, T. M. Fernández-Caramés, M. Suárez-Albela, L. Castedo, and M. González-López, "A review on Internet of Things for defense and public safety," Sensors, vol. 16, no. 10, 2016.

[158] B. Zhang, C. H. Liu, J. Tang, Z. Xu, J. Ma, and W. Wang, "Learningbased energy-efficient data collection by unmanned vehicles in smart cities," IEEE Transactions on Industrial Informatics, vol. 14, no. 4, pp. 1666-1676, Apr 2018.

[159] Z. Zhou, J. Feng, B. Gu, B. Ai, S. Mumtaz, J. Rodriguez, and M. Guizani, "When mobile crowd sensing meets UAV: Energyefficient task assignment and route planning," IEEE Transactions on Communications, vol. 66, no. 11, pp. 5526-5538, Nov 2018.

[160] E. Barka, C. A. Kerrache, N. Lagraa, and A. Lakas, "Behavioraware UAV-assisted crowd sensing technique for urban vehicular environments," in 15th IEEE Annual Consumer Communications Networking Conference (CCNC), Jan 2018, pp. 1-7.

[161] Y. Zheng, L. Capra, O. Wolfson, and H. Yang, "Urban computing Concepts, methodologies, and applications," ACM Transactions on Intelligent Systems and Technology, vol. 5, no. 3, pp. 1-55, Sep 2014

[162] L. Summers and S. D. Johnson, "Does the configuration of the street network influence where outdoor serious violence takes place? using space syntax to test crime pattern theory," Journal of Quantitative Criminology, vol. 33, no. 2, pp. 397-420, Jun 2017.

[163] W. Guo and S. Wang, "Mobile crowd-sensing wireless activity with measured interference power," IEEE Wireless Communications Letters, vol. 2, no. 5, pp. 539-542, 2013.

[164] A. Farshad, M. K. Marina, and F. Garcia, "Urban WiFi characterization via mobile crowdsensing," in IEEE Network Operations and Management Symposium (NOMS), 2014, pp. 1-9.

[165] S. Rosen, S.-J. Lee, J. Lee, P. Congdon, Z. M. Mao, and K. Burden, "MCNet: Crowdsourcing wireless performance measurements through the eyes of mobile devices," IEEE Communications Magazine, vol. 52, no. 10, pp. 86-91, 2014.

[166] H. Lu, W. Pan, N. D. Lane, T. Choudhury, and A. T. Campbell, "SoundSense: scalable sound sensing for people-centric applications on mobile phones," in Proceedings of the ACM International Conference on Mobile Systems, Applications, and Services, 2009, pp. 165-178.

[167] V. Subbaraju, A. Kumar, V. Nandakumar, S. Batra, S. Kanhere, P. De, V. Naik, D. Chakraborty, and A. Misra, "ConferenceSense: monitoring of public events using phone sensors," in Proc. of the ACM Conference on Pervasive and Ubiquitous Computing, 2013, pp. 1167-1174.

[168] M. Talasila, R. Curtmola, and C. Borcea, "Improving location reliability in crowd sensed data with minimal efforts," in IFIP Wireless and Mobile Networking Conference (WMNC), 2013, pp. 1-8.

[169] Z. Yu, Y. Feng, H. Xu, and X. Zhou, "Recommending travel packages based on mobile crowdsourced data," IEEE Communications Magazine, vol. 52 , no. 8 , pp. 56-62, 2014.

[170] H. Habibzadeh, T. Soyata, B. Kantarci, A. Boukerche, and C. Kaptan, "Sensing, communication and security planes: A new challenge for a smart city system design," Computer Networks, vol. 144, pp. 163 - 200, 2018.

[171] T. Anagnostopoulos, A. Zaslavsky, K. Kolomvatsos, A. Medvedev, P. Amirian, J. Morley, and S. Hadjieftymiades, "Challenges and opportunities of waste management in IoT-enabled smart cities: A survey," IEEE Transactions on Sustainable Computing, vol. 2, no. 3, pp. 275-289, Jul 2017.

[172] P. P. Jayaraman, A. Sinha, W. Sherchan, S. Krishnaswamy, A. Zaslavsky, P. D. Haghighi, S. Loke, and M. T. Do, "Here-n-now: A framework for context-aware mobile crowdsensing," in Proceedings of the International Conference on Pervasive Computing, 2012.

[173] J. Chon and H. Cha, "Lifemap: A smartphone-based context provider for location-based services," IEEE Pervasive Computing, no. 2, pp. $58-67,2011$.

[174] N. D. Lane, Y. Chon, L. Zhou, Y. Zhang, F. Li, D. Kim, G. Ding, F. Zhao, and H. Cha, "Piggyback crowdsensing (PCS): energy efficient crowdsourcing of mobile sensor data by exploiting smartphone app opportunities," in Proc. of the ACM Conference on Embedded Networked Sensor Systems, ser. SenSys, 2013.

[175] A. Capponi, C. Fiandrino, D. Kliazovich, P. Bouvry, and S. Giordano, "A cost-effective distributed framework for data collection in cloud-based mobile crowd sensing architectures," IEEE Transactions on Sustainable Computing, vol. 2, no. 1, pp. 3-16, Jan 2017.

[176] F. Montori, L. Bedogni, and L. Bononi, "Distributed data collection control in opportunistic mobile crowdsensing," in Proc. of the ACM Workshop on Experiences with the Design and Implementation of Smart Objects, ser. SMARTOBJECTS, 2017, pp. 19-24.

[177] H. Xiong, D. Zhang, L. Wang, J. P. Gibson, and J. Zhu, "EEMC: Enabling energy-efficient mobile crowdsensing with anonymous participants," ACM Transactions on Intelligent Systems and Technology (TIST), vol. 6, no. 3, pp. 1-26, Apr. 2015. [Online]. Available: http://doi.acm.org.proxy.bnl.lu/10.1145/2644827

[178] J. Wang, Y. Wang, D. Zhang, and S. Helal, "Energy saving techniques in mobile crowd sensing: Current state and future opportunities," IEEE Communications Magazine, vol. 56, no. 5, pp. 164-169, May 2018.

[179] C. Wietfeld, C. Ide, and B. Dusza, "Resource efficient mobile communications for crowd-sensing," in ACM/EDAC/IEEE Design Automation Conference (DAC), 2014, pp. 1-6.

[180] A. Chatterjee, M. Borokhovich, L. R. Varshney, and S. Vishwanath, "Efficient and flexible crowdsourcing of specialized tasks with precedence constraints," in IEEE Conference on Computer Communications (INFOCOM), Apr 2016, pp. 1-9.

[181] F. Montori, L. Bedogni, A. D. Chiappari, and L. Bononi, "SenSquare: A mobile crowdsensing architecture for smart cities," in 2016 IEEE $3 r d$ World Forum on Internet of Things (WF-IoT), Dec 2016, pp. 536-541.

[182] A. Zaslavsky, P. P. Jayaraman, and S. Krishnaswamy, "Sharelikescrowd: Mobile analytics for participatory sensing and crowd-sourcing applications," in IEEE International Conference on Data Engineering Workshops (ICDEW), 2013, pp. 128-135.

[183] Y. Xiao, P. Simoens, P. Pillai, K. Ha, and M. Satyanarayanan, "Lowering the barriers to large-scale mobile crowdsensing," in Proc. of the ACM Workshop on Mobile Computing Systems and Applications, 2013, p. 9.

[184] Q. Zhu, M. Y. S. Uddin, N. Venkatasubramanian, and C. Hsu, "Spatiotemporal scheduling for crowd augmented urban sensing," in IEEE Conference on Computer Communications (INFOCOM), Apr 2018, pp. 1997-2005.

[185] A. Khan, S. K. A. Imon, and S. K. Das, "Ensuring energy efficient coverage for participatory sensing in urban streets," in IEEE International Conference on Smart Computing (SMARTCOMP), 2014, pp. 167-174.

[186] V. I. Nistorica, C. Chilipirea, and C. Dobre, "How many people are needed for a crowdsensing campaign?" in IEEE 12th International Conference on Intelligent Computer Communication and Processing (ICCP), Sep 2016, pp. 353-358

[187] L. Wang, D. Zhang, Y. Wang, C. Chen, X. Han, and A. M'hamed, "Sparse mobile crowdsensing: challenges and opportunities," IEEE Communications Magazine, vol. 54, no. 7, pp. 161-167, July 2016.

[188] X. Tang, C. Wang, X. Yuan, and Q. Wang, "Non-interactive privacypreserving truth discovery in crowd sensing applications," in IEEE Conference on Computer Communications (INFOCOM), Apr 2018.

[189] L. Cheng, J. Niu, L. Kong, C. Luo, Y. Gu, W. He, and S. K. Das, "Compressive sensing based data quality improvement for crowd-sensing applications," Journal of Network and Computer Applications, vol. 77, pp. $123-134,2017$.

[190] M. Xiao, J. Wu, S. Zhang, and J. Yu, "Secret-sharing-based secure user recruitment protocol for mobile crowdsensing," in IEEE Conference on Computer Communications (INFOCOM), May 2017, pp. 1-9. 
[191] J. Lin, M. Li, D. Yang, and G. Xue, "Sybil-proof online incentive mechanisms for crowdsensing," in IEEE Conference on Computer Communications (INFOCOM), Apr 2018.

[192] D. Wu, S. Si, S. Wu, and R. Wang, "Dynamic trust relationships aware data privacy protection in mobile crowd-sensing," IEEE Internet of Things Journal, vol. 5, no. 4, pp. 2958-2970, Aug 2018

[193] S. Bhattacharjee, N. Ghosh, V. K. Shah, and S. K. Das, "QnQ: Quality and quantity based unified approach for secure and trustworthy mobile crowdsensing," IEEE Transactions on Mobile Computing, Dec 2018.

[194] A. Mehrotra, S. R. Müller, G. M. Harari, S. D. Gosling, C. Mascolo, M. Musolesi, and P. J. Rentfrow, "Understanding the role of places and activities on mobile phone interaction and usage patterns," Proceedings of the ACM on Interactive, Mobile, Wearable and Ubiquitous Technologies, vol. 1, no. 3, p. 84, 2017.

[195] W. Wu, J. Wang, M. Li, K. Liu, F. Shan, and J. Luo, "Energy-efficient transmission with data sharing in participatory sensing systems," IEEE Journal on Selected Areas in Communications, vol. 34, no. 12, pp 4048-4062, Dec 2016.

[196] J. Wang, J. Tang, G. Xue, and D. Yang, "Towards energy-efficient task scheduling on smartphones in mobile crowd sensing systems," Computer Networks, vol. 115, no. Supplement C, pp. 100 - 109, 2017.

[197] Q. Zhao, Y. Zhu, H. Zhu, J. Cao, G. Xue, and B. Li, "Fair energy-efficient sensing task allocation in participatory sensing with smartphones," in IEEE Conference on Computer Communications (INFOCOM), Apr 2014, pp. 1366-1374.

[198] Z. Zheng, F. Wu, X. Gao, H. Zhu, S. Tang, and G. Chen, "A budget feasible incentive mechanism for weighted coverage maximization in mobile crowdsensing," IEEE Transactions on Mobile Computing, vol. 16, no. 9, pp. 2392-2407, Sep 2017.

[199] A. Obinikpo, Y. Zhang, H. Song, T. H. Luan, and B. Kantarci, "Queuing algorithm for effective target coverage in mobile crowd sensing," IEEE Internet of Things Journal, vol. 4, no. 4, pp. 1046-1055, Aug 2017.

[200] S. He, D. H. Shin, J. Zhang, and J. Chen, "Near-optimal allocation algorithms for location-dependent tasks in crowdsensing," IEEE Transactions on Vehicular Technology, vol. 66, no. 4, pp. 3392-3405, Apr 2017.

[201] A. Ahmed, K. Yasumoto, Y. Yamauchi, and M. Ito, "Distance and time based node selection for probabilistic coverage in people-centric sensing," in IEEE Conference on Sensor, Mesh and Ad Hoc Communications and Networks, Jun 2011, pp. 134-142.

[202] M. Xiao, J. Wu, H. Huang, L. Huang, and C. Hu, "Deadlinesensitive user recruitment for mobile crowdsensing with probabilistic collaboration," in IEEE International Conference on Network Protocols (ICNP), Nov 2016, pp. 1-10.

[203] K. Han, C. Zhang, J. Luo, M. Hu, and B. Veeravalli, "Truthful scheduling mechanisms for powering mobile crowdsensing," IEEE Transactions on Computers, vol. 65, no. 1, pp. 294-307, Jan 2016.

[204] B. Liu, C. Song, M. Liu, and N. Liu, "Distinguishing uncertain objects with multiple features for crowdsensing," in IEEE Global Communications Conference (GLOBECOM), 2014, pp. 2751-2756.

[205] T. Zhou, B. Xiao, Z. Cai, M. Xu, and X. Liu, "From uncertain photos to certain coverage: A novel photo selection approach to mobile crowdsensing," in IEEE Conference on Computer Communications (INFOCOM), Apr 2018

[206] J. Li, Y. Zhu, and J. Yu, "Load balance vs utility maximization in mobile crowd sensing: A distributed approach," in IEEE Global Communications Conference (GLOBECOM), 2014, pp. 265-270.

[207] L. Wang, Z. Yu, B. Guo, F. Yi, and F. Xiong, "Mobile crowd sensing task optimal allocation: a mobility pattern matching perspective," Frontiers of Computer Science, vol. 12, no. 2, pp. 231-244, Apr 2018.

[208] H. Xiong, D. Zhang, G. Chen, L. Wang, V. Gauthier, and L. E. Barnes, "iCrowd: Near-optimal task allocation for piggyback crowdsensing," IEEE Transactions on Mobile Computing, vol. 15, no. 8, pp. 20102022, Aug 2016

[209] Y. Liu, B. Guo, Y. Wang, W. Wu, Z. Yu, and D. Zhang, "Taskme: Multitask allocation in mobile crowd sensing," in Proceedings of the ACM International Joint Conference on Pervasive and Ubiquitous Computing, ser. UbiComp, 2016, pp. 403-414.

[210] M. Xiao, J. Wu, L. Huang, Y. Wang, and C. Liu, "Multi-task assignment for crowdsensing in mobile social networks," in IEEE Conference on Computer Communications (INFOCOM), Apr 2015, pp. 2227-2235.

[211] W. Sun, Y. Zhu, L. M. Ni, and B. Li, "Crowdsourcing sensing workloads of heterogeneous tasks: A distributed fairness-aware approach," in IEEE International Conference on Parallel Processing, Sep 2015, pp. 580589.

[212] Q. Zhao, Y. Zhu, H. Zhu, J. Cao, G. Xue, and B. Li, "Fair energy-efficient sensing task allocation in participatory sensing with smartphones," in IEEE Conference on Computer Communications (INFOCOM), Apr 2014, pp. 1366-1374.

[213] L. Pu, X. Chen, J. Xu, and X. Fu, "Crowdlet: Optimal worker recruitment for self-organized mobile crowdsourcing," in IEEE Conference on Computer Communications (INFOCOM), Apr 2016, pp. 1-9.

[214] L. Wang, Z. Yu, D. Zhang, B. Guo, and C. H. Liu, "Heterogeneous multi-task assignment in mobile crowdsensing using spatiotemporal correlation," IEEE Transactions on Mobile Computing, 2018.

[215] X. Wang, R. Jia, X. Tian, and X. Gan, "Dynamic task assignment in crowdsensing with location awareness and location diversity," in IEEE Conference on Computer Communications (INFOCOM), Apr 2018

[216] J. Wang, L. Wang, Y. Wang, D. Zhang, and L. Kong, "Task allocation in mobile crowd sensing: State-of-the-art and future opportunities," IEEE Internet of Things Journal, vol. 5, no. 5, pp. 3747-3757, Oct 2018.

[217] R. F. E. Khatib, N. Zorba, and H. S. Hassanein, "Cost-efficient multitasking in coverage-aware mobile crowd sensing," in International Wireless Communications Mobile Computing Conference (IWCMC), Jun 2018, pp. 594-599.

[218] H. Li, T. Li, and Y. Wang, "Dynamic participant recruitment of mobile crowd sensing for heterogeneous sensing tasks," in IEEE 12th International Conference on Mobile Ad Hoc and Sensor Systems, Oct 2015, pp. 136-144.

[219] F. Zhang, B. Jin, H. Liu, Y. W. Leung, and X. Chu, "Minimum-cost recruitment of mobile crowdsensing in cellular networks," in IEEE Global Communications Conference (GLOBECOM), Dec 2016, pp. $1-7$.

[220] M. Karaliopoulos, O. Telelis, and I. Koutsopoulos, "User recruitment for mobile crowdsensing over opportunistic networks," in IEEE Conference on Computer Communications (INFOCOM), Apr 2015, pp. 2254-2262.

[221] Z. Feng, Y. Zhu, Q. Zhang, L. M. Ni, and A. V. Vasilakos, "TRAC: Truthful auction for location-aware collaborative sensing in mobile crowdsourcing," in IEEE International Conference on Computer Communications (INFOCOM), 2014, pp. 1231-1239.

[222] D. Zhang, H. Xiong, L. Wang, and G. Chen, "CrowdRecruiter: Selecting participants for piggyback crowdsensing under probabilistic coverage constraint," in ACM International Joint Conference on Pervasive and Ubiquitous Computing, ser. UbiComp, 2014, pp. 703-714.

[223] H. Xiong, D. Zhang, G. Chen, L. Wang, and V. Gauthier, "CrowdTasker: Maximizing coverage quality in piggyback crowdsensing under budget constraint," in IEEE International Conference on Pervasive Computing and Communications (PerCom), Mar 2015, pp. 55-62.

[224] D. Yang, G. Xue, X. Fang, and J. Tang, "Incentive mechanisms for crowdsensing: Crowdsourcing with smartphones," IEEE/ACM Transactions on Networking, vol. 24, no. 3, pp. 1732-1744, Jun 2016.

[225] B. Guo, Y. Liu, W. Wu, Z. Yu, and Q. Han, "Activecrowd: A framework for optimized multitask allocation in mobile crowdsensing systems," IEEE Transactions on Human-Machine Systems, vol. 47, no. 3, pp. 392-403, Jun 2017.

[226] M. Karaliopoulos, I. Koutsopoulos, and M. Titsias, "First learn then earn: optimizing mobile crowdsensing campaigns through data-driven user profiling," in MobiHoc, 2016.

[227] D. Yang, G. Xue, X. Fang, and J. Tang, "Crowdsourcing to smartphones incentive mechanism design for mobile phone sensing," in Proceedings of the ACM International Conference on Mobile Computing and Networking, 2012, pp. 173-184.

[228] Ö. Yürür, C. H. Liu, Z. Sheng, V. C. M. Leung, W. Moreno, and K. K Leung, "Context-awareness for mobile sensing: A survey and future directions," IEEE Communications Surveys Tutorials, vol. 18, no. 1, pp. 68-93, First Quarter 2016.

[229] O. Yurur and C. H. Liu, Generic and energy-efficient context-aware mobile sensing. CRC Press, 2015.

[230] S. Taleb, H. Hajj, and Z. Dawy, "VCAMS: Viterbi-based context aware mobile sensing to trade-off energy and delay," IEEE Transactions on Mobile Computing, vol. 17, no. 1, pp. 225-242, Jan 2018.

[231] A. Ahmad, M. M. Rathore, A. Paul, W.-H. Hong, and H. Seo, "Contextaware mobile sensors for sensing discrete events in smart environment," Journal of Sensors, 2016.

[232] X. Hu, X. Li, E. C. . Ngai, V. C. M. Leung, and P. Kruchten, "Multidimensional context-aware social network architecture for mobile crowdsensing," IEEE Communications Magazine, vol. 52, no. 6, pp. 78-87, Jun 2014.

[233] P. Nguyen and K. Nahrstedt, "Context-aware crowd-sensing in opportunistic mobile social networks," in IEEE 12th International Conference on Mobile Ad Hoc and Sensor Systems, Oct 2015, pp. 477-478

[234] J. Wannenburg and R. Malekian, "Physical activity recognition from smartphone accelerometer data for user context awareness sensing," 
IEEE Transactions on Systems, Man, and Cybernetics: Systems, vol. 47, no. 12, pp. 3142-3149, Dec 2017.

[235] S. Faye, R. Frank, and T. Engel, "Adaptive activity and context recognition using multimodal sensors in smart devices," in International Conference on Mobile Computing, Applications, and Services. Springer, 2015 , pp. 33-50.

[236] H. To, L. Fan, L. Tran, and C. Shahabi, "Real-time task assignment in hyperlocal spatial crowdsourcing under budget constraints," in IEEE International Conference on Pervasive Computing and Communications (PerCom), Mar 2016, pp. 1-8.

[237] E. Wang, Y. Yang, J. Wu, W. Liu, and X. Wang, "An efficient predictionbased user recruitment for mobile crowdsensing," IEEE Transactions on Mobile Computing, vol. 17, no. 1, pp. 16-28, Jan 2018.

[238] Q. Xu and R. Zheng, "When data acquisition meets data analytics: A distributed active learning framework for optimal budgeted mobile crowdsensing," in IEEE Conference on Computer Communications (INFOCOM), 2017, pp. 1-9.

[239] B. Song, H. Shah-Mansouri, and V. W. S. Wong, "Quality of sensing aware budget feasible mechanism for mobile crowdsensing," IEEE Transactions on Wireless Communications, vol. 16, no. 6, pp. 36193631, Jun 2017.

[240] Y. Qu, S. Tang, C. Dong, P. Li, S. Guo, H. Dai, and F. Wu, "Posted pricing for chance constrained robust crowdsensing," IEEE Transactions on Mobile Computing, pp. 1-1, 2018.

[241] Z. He, J. Cao, and X. Liu, "High quality participant recruitment in vehicle-based crowdsourcing using predictable mobility," in IEEE Conference on Computer Communications (INFOCOM), Apr 2015, pp. 2542-2550.

[242] G. Cardone, A. Corradi, L. Foschini, and R. Ianniello, "ParticipAct: A large-scale crowdsensing platform," IEEE Transactions on Emerging Topics in Computing, vol. 4, no. 1, pp. 21-32, Jan 2016.

[243] R. Rouvoy, “APISENSE: Mobile crowd-sensing made easy!” Jun. 2016 keynote of ECAAS'16. [Online]. Available: https://hal.inria.fr/hal01332594

[244] "SenseMyCity: Crowdsourcing an urban sensor," 2014. [Online]. Available: http://cloud.futurecities.up.pt/sensemycity/

[245] F. Kalim, J. P. Jeong, and M. U. Ilyas, "CRATER: A crowd sensing application to estimate road conditions," IEEE Access, vol. 4, pp. 8317$8326,2016$.

[246] M.-R. Ra, B. Liu, T. F. La Porta, and R. Govindan, "Medusa: A programming framework for crowd-sensing applications," in Proceedings of the ACM International Conference on Mobile Systems, Applications, and Services, 2012, pp. 337-350.

[247] T. Das, P. Mohan, V. N. Padmanabhan, R. Ramjee, and A. Sharma, "PRISM: platform for remote sensing using smartphones," in Proceedings of the ACM International Conference on Mobile Systems, Applications, and Services, 2010, pp. 63-76.

[248] I. Carreras, D. Miorandi, A. Tamilin, E. R. Ssebaggala, and N. Conci, "Matador: Mobile task detector for context-aware crowd-sensing campaigns," in IEEE International Conference on Pervasive Computing and Communications Workshops (PERCOM Workshops), Mar 2013, pp. 212-217.

[249] K. Mehdi, M. Lounis, A. Bounceur, and T. Kechadi, "CupCarbon: A multi-agent and discrete event wireless sensor network design and simulation tool," in International ICST Conference on Simulation Tools and Techniques, ser. SIMUTools, 2014, pp. 126-131.

[250] K. Farkas and I. Lendák, "Simulation environment for investigating crowd-sensing based urban parking," in International Conference on Models and Technologies for Intelligent Transportation Systems (MTITS), Jun 2015, pp. 320-327.

[251] J. Scott, R. Gass, J. Crowcroft, P. Hui, C. Diot, and A. Chaintreau, "CRAWDAD dataset cambridge/haggle (v. 2009-05-29)," Downloaded from http://crawdad.org/cambridge/haggle/20090529, May 2009.

[252] N. Eagle and A. (Sandy) Pentland, "Reality mining: Sensing complex social systems," Personal Ubiquitous Comput., vol. 10, no. 4, pp. 255 268, Mar. 2006.

[253] N. Kiukkonen, B. J., O. Dousse, D. Gatica-Perez, and J. K. Laurila, "Towards rich mobile phone datasets: Lausanne data collection campaign," in ACM International Conference on Pervasive Services (ICPS), Jul 2010.

[254] L. Aoude, Z. Dawy, S. Sharafeddine, K. Frenn, and K. Jahed, "Socialaware device-to-device offloading based on experimental mobility and content similarity models," Wireless Communications and Mobile Computing, 2018.

[255] Y. Xiao, P. Simoens, P. Pillai, K. Ha, and M. Satyanarayanan, "Lowering the barriers to large-scale mobile crowdsensing," in Proc. of the
ACM Workshop on Mobile Computing Systems and Applications, ser. HotMobile, 2013, pp. 1-6.

[256] K. Farkas and I. Lendák, "Evaluation of simulation engines for crowdsensing activities," in International Conference \& Workshop Mechatronics in Practice and Education, ser. MECHEDU, 2015, pp. 126-131.

[257] G. Cacciatore, C. Fiandrino, D. Kliazovich, F. Granelli, and P. Bouvry, "Cost analysis of smart lighting solutions for smart cities," in IEEE International Conference on Communications (ICC), May 2017, pp. $1-6$.

[258] S. Chessa, M. Girolami, L. Foschini, R. Ianniello, A. Corradi, and P. Bellavista, "Mobile crowd sensing management with the ParticipAct living lab," Pervasive and Mobile Computing, pp. -, 2016.

[259] Z. Zheng, Y. Peng, F. Wu, S. Tang, and G. Chen, "Trading data in the crowd: Profit-driven data acquisition for mobile crowdsensing," IEEE Journal on Selected Areas in Communications, vol. 35, no. 2, pp. 486-501, Feb 2017.

[260] M. Dai, Z. Su, Y. Wang, and Q. Xu, "Contract theory based incentive scheme for mobile crowd sensing networks," in Proc. MoWNeT, Jun 2018, pp. $1-5$.

[261] L. Duan, T. Kubo, K. Sugiyama, J. Huang, T. Hasegawa, and J. Walrand, "Incentive mechanisms for smartphone collaboration in data acquisition and distributed computing," in Proceedings IEEE INFOCOM, Mar 2012, pp. 1701-1709.

[262] C. Jiang, L. Gao, L. Duan, and J. Huang, "Scalable mobile crowdsensing via Peer-to-Peer data sharing," IEEE Transactions on Mobile Computing, vol. 17, no. 4, pp. 898-912, Apr 2018.

[263] X. Zeng, L. Gao, C. Jiang, T. Wang, J. Liu, and B. Zou, "A hybrid pricing mechanism for data sharing in P2P-based mobile crowdsensing," in 16th International Symposium on Modeling and Optimization in Mobile, Ad Hoc, and Wireless Networks (WiOpt), May 2018, pp. 1-8.

[264] Y. Li, C. A. Courcoubetis, and L. Duan, "Dynamic routing for social information sharing," IEEE Journal on Selected Areas in Communications, vol. 35, no. 3, pp. 571-585, Mar 2017.

[265] M. H. Cheung, F. Hou, and J. Huang, "Delay-sensitive mobile crowdsensing: Algorithm design and economics," IEEE Transactions on Mobile Computing, vol. 17, no. 12, pp. 2761-2774, Dec 2018.

[266] C. Jiang, L. Gao, L. Duan, and J. Huang, "Economics of Peer-to-Peer mobile crowdsensing," in IEEE Global Communications Conference (GLOBECOM), Dec 2015, pp. 1-6.

[267] Q. Ma, L. Gao, Y. Liu, and J. Huang, "Incentivizing Wi-Fi network crowdsourcing: A contract theoretic approach," IEEE/ACM Transactions on Networking, vol. 26, no. 3, pp. 1035-1048, Jun 2018.

[268] L. Shu, Y. Chen, Z. Huo, N. Bergmann, and L. Wang, "When mobile crowd sensing meets traditional industry," IEEE Access, vol. 5, pp. $15300-15307,2017$.

[269] N. Haderer, R. Rouvoy, and L. Seinturier, "A preliminary investigation of user incentives to leverage crowdsensing activities," in IEEE International Conference on Pervasive Computing and Communications Workshops (PERCOM Workshops), 2013, pp. 199-204.

[270] T. Luo, H. P. Tan, and L. Xia, "Profit-maximizing incentive for participatory sensing," in IEEE Conference on Computer Communications (INFOCOM), Apr 2014, pp. 127-135.

[271] I. Koutsopoulos, "Optimal incentive-driven design of participatory sensing systems," in IEEE Conference on Computer Communications (INFOCOM), Apr 2013, pp. 1402-1410.

[272] J. Sun, "Incentive mechanisms for mobile crowd sensing: Current states and challenges of work," arXiv preprint arXiv:1310.8364, 2013.

[273] M. Pouryazdan, C. Fiandrino, B. Kantarci, T. Soyata, D. Kliazovich, and P. Bouvry, "Intelligent gaming for mobile crowd-sensing participants to acquire trustworthy big data in the Internet of Things," IEEE Access, vol. 5, pp. 22 209-22 223, Oct 2017.

[274] T. Tsujimori, N. Thepvilojanapong, Y. Ohta, H. Wang, Y. Zhao, and Y. Tobe, "SenseUtil: Utility vs. incentives in participatory sensing," in Proc. IEICE Workshop on Smart Sens., Wireless Commun., Human Probes, 2013, pp. 24-29.

[275] D. Zhao, X. Y. Li, and H. Ma, "Budget-feasible online incentive mechanisms for crowdsourcing tasks truthfully," IEEE/ACM Transactions on Networking, vol. 24, no. 2, pp. 647-661, Apr 2016.

[276] S. Reddy, D. Estrin, and M. Srivastava, "Recruitment framework for participatory sensing data collections," in Pervasive Computing. Springer, 2010, pp. 138-155.

[277] C. Fiandrino, F. Anjomshoa, B. Kantarci, D. Kliazovich, P. Bouvry, and J. N. Matthews, "Sociability-driven framework for data acquisition in mobile crowdsensing over fog computing platforms for smart cities," IEEE Transactions on Sustainable Computing, vol. 2, no. 4, pp. 345-358, Oct 2017. 
[278] M. Marjanović, A. Antonić, and I. P. Žarko, "Edge computing architecture for mobile crowdsensing," IEEE Access, vol. 6, pp. 10662 10674,2018

[279] P. Vitello, A. Capponi, C. Fiandrino, P. Giaccone, D. Kliazovich, U. Sorger, and P. Bouvry, "Collaborative data delivery for smart cityoriented mobile crowdsensing systems," in IEEE Global Communications Conference (GLOBECOM), Dec 2018, pp. 1-6.

[280] J. D. Benedetto, P. Bellavista, and L. Foschini, "Proximity discovery and data dissemination for mobile crowd sensing using LTE direct," Computer Networks, vol. 129, pp. 510 - 521, 2017.

[281] J. Weppner and P. Lukowicz, "Bluetooth based collaborative crowd density estimation with mobile phones," in IEEE International Conference on Pervasive Computing and Communications (PerCom), Mar 2013 , pp. 193-200

[282] "Bluetooth specification version 4," Bluetooth SIG, Jul 2017. [Online]. Available: https://www.bluetooth.org/docman/handlers/downloaddoc. ashx?doc_id=229737

[283] "Waze: Get the best route, every day, with real-time help from other drivers." https://www.waze.com/, 2006.

[284] H. Habibzadeh, Z. Qin, T. Soyata, and B. Kantarci, "Large-scale distributed dedicated- and non-dedicated smart city sensing systems," IEEE Sensors Journal, vol. 17, no. 23, pp. 7649-7658, Dec 2017.

[285] X. Chen, Y. Chen, M. Dong, and C. Zhang, "Demystifying energy usage in smartphones," in ACM/EDAC/IEEE Design Automation Conference (DAC), Jun 2014, pp. 1-5.

[286] N. Ravi, N. Dandekar, P. Mysore, and M. L. Littman, "Activity recognition from accelerometer data," in AAAI, vol. 5, 2005, pp. 15411546

[287] A. Bujari, B. Licar, and C. E. Palazzi, "Movement pattern recognition through smartphone's accelerometer," in IEEE Consumer communications and networking conference (CCNC), 2012, pp. 502-506.

[288] I. Shafer and M. L. Chang, "Movement detection for power-efficient smartphone WLAN localization," in Proceedings of the ACM international conference on Modeling, analysis, and simulation of wireless and mobile systems, 2010, pp. 81-90.

[289] P. Siirtola and J. Röning, "Recognizing human activities userindependently on smartphones based on accelerometer data," International Journal of Interactive Multimedia and Artificial Intelligence, vol. 1, no. 5, 2012

[290] A. Anjum and M. U. Ilyas, "Activity recognition using smartphone sensors," in IEEE Consumer Communications and Networking Conference (CCNC), 2013, pp. 914-919.

[291] M. Shoaib, H. Scholten, and P. J. Havinga, "Towards physical activity recognition using smartphone sensors," in IEEE International Conference on Ubiquitous Intelligence and Computing and on Autonomic and Trusted Computing (UIC/ATC), 2013, pp. 80-87.

[292] C. Schuss, T. Leikanger, B. Eichberger, and T. Rahkonen, "Efficient use of solar chargers with the help of ambient light sensors on smartphones," in IEEE Conference of Open Innovations Association (FRUCT), 2014 pp. 79-85.

[293] V. Freschi, S. Delpriori, E. Lattanzi, and A. Bogliolo, "Bootstrap based uncertainty propagation for data quality estimation in crowdsensing systems," IEEE Access, vol. 5, pp. 1146-1155, 2017.

[294] M. Tomasoni, A. Capponi, C. Fiandrino, D. Kliazovich, F. Granelli, and P. Bouvry, "Why energy matters? profiling energy consumption of mobile crowdsensing data collection frameworks," Pervasive and Mobile Computing, vol. 51, pp. 193 - 208, 2018. [Online]. Available: http://www.sciencedirect.com/science/article/pii/S1574119217305965

[295] X. Sheng, J. Tang, and W. Zhang, "Energy-efficient collaborative sensing with mobile phones," in IEEE Conference on Computer Communications (INFOCOM), Mar 2012, pp. 1916-1924.

[296] H. Xiong, D. Zhang, L. Wang, and H. Chaouchi, "EMC ${ }^{3}$ : Energyefficient data transfer in mobile crowdsensing under full coverage constraint," IEEE Transactions on Mobile Computing, vol. 14, no. 7, pp. 1355-1368, 2015.

[297] C. Zhang, K. Subbu, J. Luo, and J. Wu, "GROPING: Geomagnetism and crowdsensing powered indoor navigation," IEEE Transactions on Mobile Computing, vol. 14, no. 2, pp. 387-400, Feb 2015.

[298] L. Zhang, J. Liu, H. Jiang, and Y. Guan, "SensTrack: Energy-efficient location tracking with smartphone sensors," IEEE Sensors Journal, vol. 13, no. 10, pp. 3775-3784, Oct 2013.

[299] E. Ozer and M. Q. Feng, "Direction-sensitive smart monitoring of structures using heterogeneous smartphone sensor data and coordinate system transformation," Smart Materials and Structures, vol. 26, no. 4 p. 045026,2017

[300] V. M. Souza, R. Giusti, and A. J. Batista, "Asfault: A low-cost system to evaluate pavement conditions in real-time using smartphones and machine learning," Pervasive and Mobile Computing, vol. 51, pp. 121 137, 2018. [Online]. Available: http://www.sciencedirect.com/science/ article/pii/S1574119218300518

[301] T. Ludwig, C. Reuter, T. Siebigteroth, and V. Pipek, "CrowdMonitor: Mobile crowd sensing for assessing physical and digital activities of citizens during emergencies," in Proc. of the ACM Conference on Human Factors in Computing Systems, ser. SIGCHI, 2015, pp. 4083-4092.

[302] N. B. Grimm, S. H. Faeth, N. E. Golubiewski, C. L. Redman, J. Wu, X. Bai, and J. M. Briggs, "Global change and the ecology of cities," in Science, vol. 319, no. 5864, 2008, pp. 756-760.

[303] H. B. Dulal and S. Akbar, "Greenhouse gas emission reduction options for cities: Finding the "coincidence of agendas" between local priorities and climate change mitigation objectives," Habitat International, vol. 38 , pp. $100-105,2013$

[304] A. Caragliu, C. Del Bo, and P. Nijkamp, "Smart cities in europe," Journal of urban technology, vol. 18, no. 2, pp. 65-82, 2011.

[305] A. Longo, M. Zappatore, M. Bochicchio, and S. B. Navathe, "Crowdsourced data collection for urban monitoring via mobile sensors," $A C M$ Trans. Internet Technol., vol. 18, no. 1, pp. 1-21, Oct 2017.

[306] Y. Zhou, B. P. L. Lau, C. Yuen, B. Tunçer, and E. Wilhelm, "Understand urban human mobility through crowdsensed data," CoRR, vol. abs/1805.00628, 2018

[307] M. S. Kaiser, K. T. Lwin, M. Mahmud, D. Hajializadeh, T. Chaipimonplin, A. Sarhan, and M. A. Hossain, "Advances in crowd analysis for urban applications through urban event detection," IEEE Transactions on Intelligent Transportation Systems, pp. 1-21, 2017.

[308] "Cisco global cloud index: Forecast and methodology, 2016-2021," Cisco Visual Networking, 2018, White Paper.

[309] X. Cheng, L. Fang, X. Hong, and L. Yang, "Exploiting mobile big data: Sources, features, and applications," IEEE Network, vol. 31, no. 1, pp. 72-79, Jan 2017.

[310] Y. Sun, H. Song, A. J. Jara, and R. Bie, "Internet of Things and big data analytics for smart and connected communities," IEEE Access, vol. 4, pp. 766-773, 2016

[311] C. Zhang, P. Patras, and H. Haddadi, "Deep learning in mobile and wireless networking: A survey," CoRR, vol. abs/1803.04311, 2018. [Online]. Available: http://arxiv.org/abs/1803.04311

[312] J. Wang, Y. Wang, D. Zhang, J. Goncalves, D. Ferreira, A. Visuri, and S. Ma, "Learning-assisted optimization in mobile crowd sensing: A survey," IEEE Transactions on Industrial Informatics, vol. 15, no. 1 , pp. 15-22, Jan 2019

[313] Y. LeCun, Y. Bengio, and G. Hinton, "Deep learning," Nature, vol. 521, no. 7553 , p. $436,2015$.

[314] A. Dubey, N. Naik, D. Parikh, R. Raskar, and C. A. Hidalgo, "Deep learning the city: Quantifying urban perception at a global scale," in Computer Vision - ECCV. Springer International Publishing, 2016, pp. 196-212.

[315] "Foobot: Better air, better life," 2017. [Online]. Available: https: //foobot.io/

[316] Y. Lv, Y. Duan, W. Kang, Z. Li, and F. Y. Wang, "Traffic flow prediction with big data: A deep learning approach," IEEE Transactions on Intelligent Transportation Systems, vol. 16, no. 2, pp. 865-873, Apr 2015 .

[317] "Dangerous by design," 2011. [Online]. Available: http://t4america.org/ docs/dbd2011/Dangerous-by-Design-2011.pdf

[318] L. Wang, D. Zhang, D. Yang, A. Pathak, C. Chen, X. Han, H. Xiong, and Y. Wang, "SPACE-TA: Cost-effective task allocation exploiting intradata and interdata correlations in sparse crowdsensing," ACM Trans. Intell. Syst. Technol., vol. 9, no. 2, pp. 1-20, Oct 2017.

[319] L. Fu, S. Ma, L. Kong, S. Liang, and X. Wang, "FINE: A framework for distributed learning on incomplete observations for heterogeneous crowdsensing networks," IEEE/ACM Transactions on Networking, vol. 26, no. 3, pp. 1092-1109, Jun 2018.

[320] S. Yang, K. Han, Z. Zheng, S. Tang, and F. Wu, "Towards personalized task matching in mobile crowdsensing via fine-grained user profiling," in IEEE Conference on Computer Communications (INFOCOM), Apr 2018

[321] K. Christidis and M. Devetsikiotis, "Blockchains and smart contracts for the Internet of Things," IEEE Access, vol. 4, pp. 2292-2303, 2016

[322] "Hyperledger," 2016. [Online]. Available: https://www.hyperledger.org/

[323] G. Wood, "Ethereum: A secure decentralised generalised transaction ledger," Ethereum project yellow paper, vol. 151, pp. 1-32, 2014.

[324] P. Hu, S. Dhelim, H. Ning, and T. Qiu, "Survey on fog computing: architecture, key technologies, applications and open issues," Journal of Network and Computer Applications, vol. 98, pp. 27 - 42, 2017. 
[325] C. Fiandrino, N. Allio, D. Kliazovich, P. Giaccone, and P. Bouvry, "Profiling performance of application partitioning for wearable devices in mobile cloud and fog computing," IEEE Access, vol. 7, pp. 12156 12 166, Jan 2019.

[326] P. P. Jayaraman, J. B. Gomes, H. L. Nguyen, Z. S. Abdallah, S. Krishnaswamy, and A. Zaslavsky, "Scalable energy-efficient distributed data analytics for crowdsensing applications in mobile environments," IEEE Trans. on Computational Social Systems, vol. 2/3, pp. 109-123, Sep 2015.

[327] P. Bellavista, S. Chessa, L. Foschini, L. Gioia, and M. Girolami, "Humanenabled edge computing: Exploiting the crowd as a dynamic extension of mobile edge computing," IEEE Communications Magazine, vol. 56, no. 1 , pp. 145-155, Jan 2018.

[328] R. Roman, J. Lopez, and M. Mambo, "Mobile edge computing, fog et al.: A survey and analysis of security threats and challenges," Future Generation Computer Systems, vol. 78, pp. 680 - 698, 2018

[329] F. Bonomi, R. Milito, J. Zhu, and S. Addepalli, "Fog computing and its role in the Internet of Things," in Proceedings of the ACM Workshop on Mobile Cloud Computing, ser. MCC, 2012, pp. 13-16.

[330] T. Taleb, K. Samdanis, B. Mada, H. Flinck, S. Dutta, and D. Sabella, "On multi-access edge computing: A survey of the emerging 5G network edge cloud architecture and orchestration," IEEE Communications Surveys Tutorials, vol. 19, no. 3, pp. 1657-1681, Third Quarter 2017.

[331] Z. Zhou, H. Liao, B. Gu, K. M. S. Huq, S. Mumtaz, and J. Rodriguez, "Robust mobile crowd sensing: When deep learning meets edge computing," IEEE Network, vol. 32, no. 4, pp. 54-60, Jul 2018.

[332] S. Yang, J. Bian, L. Wang, H. Zhu, Y. Fu, and H. Xiong, "Edgesense: Edge-mediated spatial-temporal crowdsensing," IEEE Access, pp. 1-1, 2018.

[333] M. Shafi, A. F. Molisch, P. J. Smith, T. Haustein, P. Zhu, P. D. Silva, F. Tufvesson, A. Benjebbour, and G. Wunder, "5G: A tutorial overview of standards, trials, challenges, deployment, and practice," IEEE Journal on Selected Areas in Communications, vol. 35, no. 6, pp. 1201-1221, Jun 2017.

[334] M. Simsek, A. Aijaz, M. Dohler, J. Sachs, and G. Fettweis, "5G-Enabled Tactile Internet," IEEE Journal on Selected Areas in Communications, vol. 34, no. 3, pp. 460-473, Mar 2016.

[335] C. Fiandrino, H. Assasa, P. Casari, and J. Widmer, "Scaling millimeterwave networks to dense deployments and dynamic environments," Proceedings of the IEEE, vol. 107, no. 4, pp. 732-745, Apr 2019.

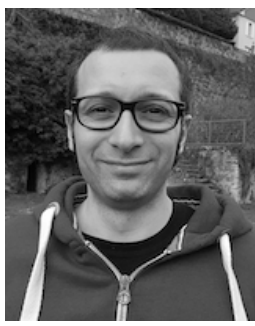

Andrea Capponi (S'16) is a Ph.D. candidate at the University of Luxembourg. He received the Bachelor Degree and the Master Degree both in Telecommunication Engineering from the University of Pisa, Italy. His research interests are mobile crowdsensing, urban computing, and IoT.

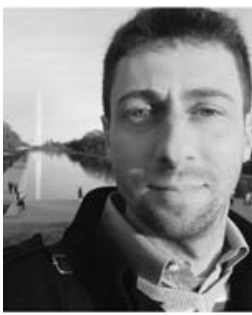

Claudio Fiandrino (S'14-M'17) is a postdoctoral researcher at IMDEA Networks. He received his Ph.D. degree at the University of Luxembourg (2016), the Bachelor Degree (2010) and Master Degree (2012) both from Politecnico di Torino (Italy). Claudio was awarded with the Spanish Juan de la Cierva grant and the Best Paper Awards in IEEE CloudNet'16 and in ACM WiNTECH'18. His research interests include mobile crowdsensing, edge computing and URLLC.

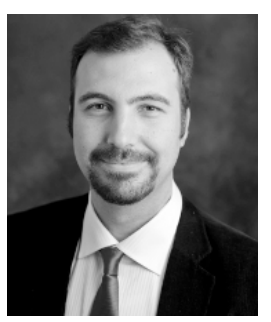

Burak Kantarci (S'05-M'09-SM'12) is an Associate Professor and the founding director of the Next Generation Communications and Computing Networks (NEXTCON) Research laboratory in the School of Electrical Engineering and Computer Science at the University of Ottawa (Canada). He received the M.Sc. and Ph.D. degrees in computer engineering from Istanbul Technical University in 2005 and 2009, respectively. He is an Area Editor of the IEEE TRANSACTIONS ON GREEN COMMUNICATIONS NETWORKING, an Associate Editor of the IEEE COMmunications Surveys AND TUtorials, and an Associate Editor of the IEEE ACCESS and IEEE Networking Letters. He also serves as the Chair of the IEEE ComSoc Communication Systems Integration and Modeling Technical Committee. Dr. Kantarci is a senior member of the IEEE member of the ACM, and a distinguished speaker of the ACM.

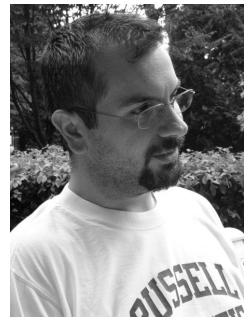

IEEE
Luca Foschini (M'04-SM'19) graduated from the University of Bologna, from which he received a $\mathrm{Ph} . \mathrm{D}$. degree in Computer Engineering in 2007. He is now an Assistant Professor of Computer Engineering at the University of Bologna. His interests include distributed systems and solutions for system and service management, management of cloud computing, context data distribution platforms for smart city scenarios, context-aware session control, mobile edge computing, and mobile crowdsensing and crowdsourcing. He is a member of ACM and

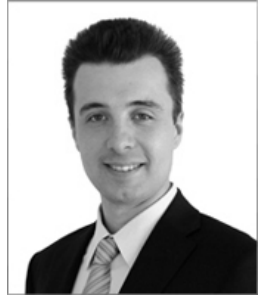

Dzmitry Kliazovich (M'03-SM'12) is a Head of Innovation at ExaMotive. Dr. Kliazovich holds an award-winning Ph.D. in Information and Telecommunication Technologies from the University of Trento (Italy). He coordinated organization and chaired a number of highly ranked international conferences and symposia. Dr. Kliazovich is a frequent keynote and panel speaker. He is Associate Editor of IEEE Communications Surveys and Tutorials and of IEEE Transactions of Cloud Computing. His main research activities are in intelligent and shared mobility, energy efficiency, cloud systems and architectures, data analytics and IoT.

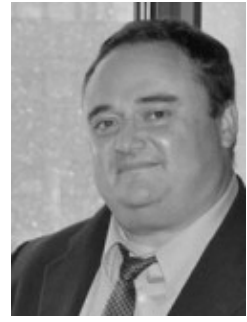

Pascal Bouvry is a professor at the Faculty of Science, Technology and Communication at the University of Luxembourg and faculty member at SnT Center. Prof. Bouvry has a Ph.D. in computer science from the University of Grenoble (INPG), France. He is on the IEEE Cloud Computing and Elsevier Swarm and Evolutionary Computation editorial boards, communication vice-chair of the IEEE STC on Sustainable Computing and co-founder of the IEEE TC on Cybernetics for Cyber-Physical Systems. His research interests include cloud \& parallel computing, optimization, security and reliability. 